\title{
Search for resonances decaying into a weak vector boson and a Higgs boson in the fully hadronic final state produced in proton - proton collisions at $\sqrt{s}=13 \mathrm{TeV}$ with the ATLAS detector
}

\author{
G. Aad et al.* \\ (ATLAS Collaboration)
}

(Received 12 July 2020; accepted 29 September 2020; published 17 December 2020)

\begin{abstract}
A search for heavy resonances decaying into a $W$ or $Z$ boson and a Higgs boson produced in proton proton collisions at the Large Hadron Collider at $\sqrt{s}=13 \mathrm{TeV}$ is presented. The analysis utilizes the dominant $W \rightarrow q \bar{q}^{\prime}$ or $Z \rightarrow q \bar{q}$ and $H \rightarrow b \bar{b}$ decays with substructure techniques applied to large-radius jets. A sample corresponding to an integrated luminosity of $139 \mathrm{fb}^{-1}$ collected with the ATLAS detector is analyzed and no significant excess of data is observed over the background prediction. The results are interpreted in the context of the heavy vector triplet model with spin- $1 W^{\prime}$ and $Z^{\prime}$ bosons. Upper limits on the cross section are set for resonances with mass between 1.5 and $5.0 \mathrm{TeV}$, ranging from 6.8 to $0.53 \mathrm{fb}$ for $W^{\prime} \rightarrow W H$ and from 8.7 to $0.53 \mathrm{fb}$ for $Z^{\prime} \rightarrow Z H$ at the $95 \%$ confidence level.
\end{abstract}

DOI: $10.1103 /$ PhysRevD.102.112008

\section{INTRODUCTION}

The search for physics beyond the Standard Model (SM) is a major focus of the physics program at the Large Hadron Collider (LHC). Since its discovery [1,2], the Higgs boson has become a tool in this search. In particular, one may expect new heavy resonances to couple to Higgs bosons and weak vector bosons $(V=W$ or $Z$ ). Such resonances are expected to occur in a number of theories beyond the Standard Model. Theories that aim to solve the naturalness problem predict the existence of vector resonances as expected in composite Higgs models [3,4], Little Higgs models [5], or models with extra dimensions [6,7]. Theories with extended Higgs sectors predict scalar resonances as in two-Higgs-doublet models [8].

In this article, a search for $W H$ and $Z H$ resonances produced in proton-proton $(p p)$ collisions at $\sqrt{s}=13 \mathrm{TeV}$ is reported with a sample corresponding to an integrated luminosity of $139 \mathrm{fb}^{-1}$ collected with the ATLAS detector during Run 2 of the LHC in 2015-2018. The search is designed for resonances with a mass of at least $1.5 \mathrm{TeV}$ and with both the $V$ and $H$ bosons decaying hadronically in the modes $V \rightarrow q \bar{q}^{(\prime)}$ and $H \rightarrow b \bar{b}$, as shown in Fig. 1. In this regime, the $V$ and $H$ bosons are produced with high

${ }^{*}$ Full author list given at the end of the article.

Published by the American Physical Society under the terms of the Creative Commons Attribution 4.0 International license. Further distribution of this work must maintain attribution to the author(s) and the published article's title, journal citation, and DOI. Funded by SCOAP . transverse momentum $\left(p_{\mathrm{T}}\right)$, resulting in each boson being reconstructed as a single large-radius hadronic jet, and the invariant mass of this dijet system provides the final discriminating variable. Jet substructure techniques and $b$-tagging are then used to discriminate those jets from background jets originating from multijet, $V+$ jets, and $t \bar{t}$ events - with QCD multijet events representing at least $85 \%$ of the total background. Due to difficulties in modeling the background from simulation, all background estimates are derived from the data.

The results of the search are interpreted in the context of the heavy vector triplet (HVT) model [9], which is a simplified model providing a broad phenomenological framework for heavy resonances coupling to SM fermions and bosons. In this model, $W^{\prime}$ and $Z^{\prime}$ vector bosons interact with quarks and the Higgs field with coupling strength of $g_{q}$ and $g_{H}$, respectively. ${ }^{1}$ Coupling to the Higgs field gives rise to interactions with longitudinally polarized $W$ and $Z$ bosons. Two scenarios are considered as benchmarks for interpretation in this article. Model A corresponds to the choice $g_{q}=-0.55$ and $g_{H}=-0.56$, which reproduces the phenomenology of weakly coupled models based on an extended gauge symmetry [11]. Model B corresponds to $g_{q}=0.14$ and $g_{H}=-2.9$, which implements a strongly coupled scenario as in composite Higgs models.

Previous searches for $V H$ resonances have been carried out at $\sqrt{s}=13 \mathrm{TeV}$ in the semileptonic final state $(\nu \nu b b$, $\ell \nu b b$, and $\ell \ell b b$ ) [12-14] and fully hadronic final state $(q q b b)[15,16]$. The ATLAS and CMS collaborations

\footnotetext{
${ }^{1}$ Further details about the use of the HVT model in ATLAS analyses can be found in Ref. [10].
} 


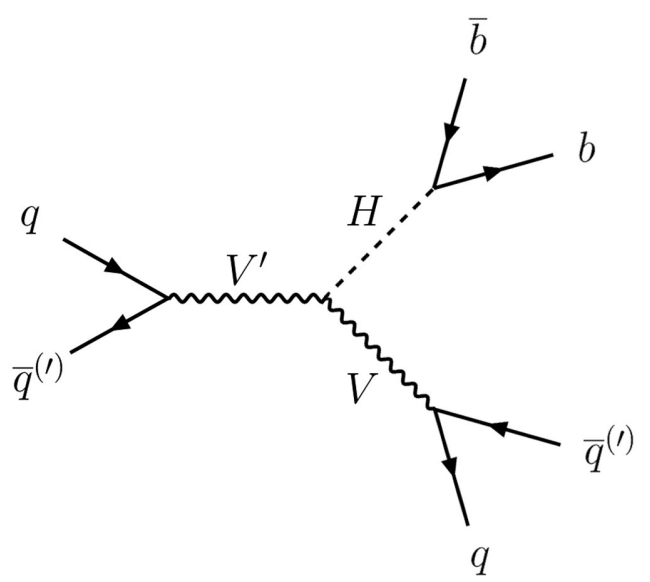

FIG. 1. Feynman diagram for the production of a $V^{\prime}$ resonance with decay into a $\mathrm{VH}$ pair.

report similar lower limits on the masses of $W^{\prime}$ and $Z^{\prime}$ bosons in these two sets of final states with about $36 \mathrm{fb}^{-1}$ of integrated luminosity collected in 2015-2016. The strongest lower limit on the $W^{\prime}$ mass is set by CMS in the $\ell \nu b b$ channel [13] with a value of $2.9 \mathrm{TeV}$ at the $95 \%$ confidence level (C.L.) in the context of HVT model B. For $Z^{\prime}$ bosons, the strongest lower limit on the mass is set by ATLAS in the combination of the $\nu \nu b b$ and $\ell \ell b b$ channels [12] with a value of $2.83 \mathrm{TeV}$ at the $95 \%$ C.L. in HVT model B.

The results presented in this article differ from those previously published by ATLAS in the $q q b b$ channel [15] thanks to the following improvements. The integrated luminosity has increased by a factor of nearly four, an improved clustering algorithm combining measurements from the calorimeter and tracking systems is used to reconstruct $V$ - and $H$-candidate jets, the $b$-tagging procedure used to identify $H$-candidate jets is performed on track jets with a $p_{\mathrm{T}}$-dependent radius that allows double $b$-tagging of $H$-candidate jets up to considerably higher $p_{\mathrm{T}}$ values, and the Higgs-candidate selection has been reoptimized with increased sensitivity.

\section{ATLAS DETECTOR}

The ATLAS experiment [17] at the LHC is a multipurpose particle detector with a forward-backward symmetric cylindrical geometry and a near $4 \pi$ coverage in solid angle. ${ }^{2}$ It consists of an inner detector (ID) for tracking surrounded

\footnotetext{
${ }^{2}$ ATLAS uses a right-handed coordinate system with its origin at the nominal interaction point (IP) in the center of the detector and the $z$ axis along the beam pipe. The $x$ axis points from the IP to the center of the LHC ring, and the $y$ axis points upwards. Cylindrical coordinates $(r, \phi)$ are used in the transverse plane, $\phi$ being the azimuthal angle around the $z$ axis. The pseudorapidity is defined in terms of the polar angle $\theta$ as $\eta=-\ln \tan (\theta / 2)$. The rapidity is defined relative to the beam axis as $y=$ $(1 / 2) \ln \left[\left(E+p_{z}\right) /\left(E-p_{z}\right)\right]$. Angular distance is measured in units of $\Delta R \equiv \sqrt{(\Delta \eta)^{2}+(\Delta \phi)^{2}}$.
}

by a thin superconducting solenoid providing a $2 \mathrm{~T}$ axial magnetic field, electromagnetic (EM) and hadronic calorimeters, and a muon spectrometer (MS). The inner detector covers the pseudorapidity range $|\eta|<2.5$. It consists of silicon pixel, silicon microstrip, and transition radiation tracking detectors. An additional innermost pixel layer $[18,19]$ inserted at a radius of $3.3 \mathrm{~cm}$ has been used since 2015. Liquid-argon (LAr) sampling calorimeters provide EM energy measurements with high granularity. A hadronic scintillator-tile calorimeter covers the central pseudorapidity range $(|\eta|<1.7)$. The end cap and forward regions are instrumented with LAr calorimeters for both the EM and hadronic energy measurements up to $|\eta|=4.9$. The muon spectrometer surrounds the calorimeters and features three large air-core toroidal superconducting magnet systems with eight coils each. The field integral of the toroids ranges between 2.0 and $6.0 \mathrm{Tm}$ across most of the detector. The muon spectrometer includes a system of precision tracking chambers and fast detectors for triggering. A two-level trigger system [20] is used to select events. The first-level trigger is implemented in hardware and uses a subset of the detector information to reduce the accepted rate to at most $100 \mathrm{kHz}$. This is followed by a softwarebased trigger level that reduces the accepted event rate to $1 \mathrm{kHz}$ on average.

\section{DATA AND MONTE CARLO SIMULATION}

The data sample for the analysis was collected by the ATLAS detector with high- $p_{\mathrm{T}}$ single-jet triggers utilizing the anti- $k_{t}$ clustering algorithm [21] with a radius $R=1.0$. The lowest unprescaled triggers were used with the following $p_{\mathrm{T}}$ thresholds: $360 \mathrm{GeV}$ in $2015,420 \mathrm{GeV}$ in 2016, and $460 \mathrm{GeV}$ in 2017-2018. After requiring that the data were recorded with stable beam conditions and satisfied detector and data quality requirements, the integrated luminosity was measured to be $139 \mathrm{fb}^{-1}$ using the methodology from Ref. [22].

The analysis relies on Monte Carlo (MC) samples to model signal events. Background MC samples are used only to optimize the signal event selection and to validate the data-driven background estimation method (Sec. V).

Signal $W^{\prime}$ and $Z^{\prime}$ events for HVT model A were produced at leading-order (LO) precision in the strong coupling constant $\left(\alpha_{\mathrm{s}}\right)$ with the MadGraph5_aMC@NLO 2.2.2 [23] event generator using the NNPDF23LO parton distribution function (PDF) set [24]. Separate generation of signal events for HVT model B is not required as both models $\mathrm{A}$ and $\mathrm{B}$ give rise to dijet mass peaks with a width that is dominated by the experimental resolution. The events were interfaced with PYTHIA 8.186 [25] for parton showering, hadronization, and the underlying event, and relied on the A14 set of tuned parameters [26]. Higgs boson decays to heavy-flavor final states $H \rightarrow b \bar{b}$ and $H \rightarrow c \bar{c}$ were included, corresponding to branching fractions of $58.2 \%$ and $2.9 \%$, respectively [27]. 
Multijet events were produced with the PYTHIA 8.186 event generator, the NNPDF23LO PDF set, and the A14 tune. Samples of events with top-quark pairs were produced at next-to-leading order (NLO) with POWHEG-BOX [28] and the NNPDF30 NLO PDF set, interfaced with PYTHIA 8.183 and the A14 tune. The $h_{\text {damp }}$ parameter was set to 1.5 times the top-quark mass [29]. Samples of $W+$ jets and $Z+$ jets events were produced with SHERPA 2.1.1 [30] and the CT10 PDF set [31] for up to two partons at NLO and up to four partons at LO. The cross sections used to normalize the multijet and the $V+$ jets MC samples were computed with PYTHIA and SHERPA, respectively, and the top-quark pair cross section was taken to be $832_{-52}^{+46} \mathrm{pb}$ for a top-quark mass of $172.5 \mathrm{GeV}$. This value was calculated at next-to-next-to-leading order in $\alpha_{\mathrm{s}}$, including the resummation of next-to-next-to-leading logarithmic soft gluon terms, with Top++2.0 [32-38]. Other SM backgrounds originating from diboson production $(V V)$ and weak vector-boson production in association with a Higgs boson $(\mathrm{VH})$ are negligible and not considered.

For all MC samples, except those produced with SHERPA, $b$-hadron and $c$-hadron decays were handled by EvtGen 1.2.0 [39]. Inelastic $p p$ events generated using PYTHIA 8.186 with the A3 tune [40] and the NNPDF23LO PDF set were added to the hard-scattering interaction in such a way as to reproduce the effects of additional $p p$ interactions (pileup) in each bunch crossing during data collection. The detector response was simulated with GEANT 4 [41,42], and the events were processed with the same reconstruction software as for the data. Energy/momentum scale and efficiency corrections are applied to the results of the simulation to account for small differences between the simulation and the performance measured directly from the data.

\section{EVENT RECONSTRUCTION AND SELECTION}

The analysis relies on the reconstruction of charged particles with $p_{\mathrm{T}}>500 \mathrm{MeV}$ in the inner detector to reconstruct $p p$ collision vertices for each crossing of the LHC beams. The primary vertex is chosen to be the vertex with the largest $\sum p_{\mathrm{T}}^{2}$ for the tracks associated with the vertex.

Jets are built from a combination of tracks and calibrated clusters of energy deposits in calorimeter cells [43], with the anti- $k_{t}$ clustering algorithm using a radius parameter $R=1.0$ as implemented in FastJet [44]. The tracks are selected with the same requirements as in Ref. [45], except for the minimum $p_{\mathrm{T}}$ value, which has been increased to $500 \mathrm{MeV}$. By combining calorimeter and tracking information, one benefits from both the better energy resolution of the calorimeter at high energy and the superior angular resolution for the tracks. This combination becomes highly beneficial at large jet $p_{\mathrm{T}}$ due to the small number of clusters produced and the limited angular resolution of the calorimeter. The resulting jets are referred to as TrackCaloCluster (TCC) jets. A detailed description of the algorithm can be found in Ref. [46] and its application to a search for high-mass diboson resonances is described in Ref. [47]. A trimming algorithm [48] is applied to minimize the impact of pileup. In this algorithm, the constituents of each jet are reclustered with the $k_{t}$ algorithm [49] into smaller $R=0.2$ subjets. Trimmed large- $R$ jets are made up of constituents of those subjets with $p_{\mathrm{T}}^{\text {subjet }} / p_{\mathrm{T}}^{\text {jet }}>$ 0.05 , where $p_{\mathrm{T}}^{\text {subjet }}$ and $p_{\mathrm{T}}^{\text {jet }}$ are the transverse momenta of the subjet and original untrimmed jet, respectively. The energy and mass calibration of TCC jets is based on the simulation as described in Ref. [50]. As a result of the improved angular resolution of the energy distribution within the jet, the discrimination between signal $W$ or $Z$ jets and background QCD jets is noticeably improved. In addition to their masses, a powerful variable to discriminate between those jets is $D_{2}$, defined as the ratio of three-point to two-point energy correlation functions that are based on the energies of the jet constituents and their pairwise angular separation [51,52]. The $D_{2}$ variable exploits the two-body structure of the $V \rightarrow q \bar{q}^{(\prime)}$ decays, absent from typical QCD jets. Another variable that provides discrimination between signal and background jets is the number of tracks $\left(n_{\text {trk }}\right)$ matched to the jets by ghost association [53]. This quantity is significantly higher for gluon-induced jets that are a component of the background than for quark-induced jets in signal events, due to the distinct energy scales involved and the different color factors for gluons and quarks.

To identify Higgs-boson jets, a separate collection of jets is built from tracks with the anti- $k_{t}$ algorithm using a $p_{\mathrm{T}^{-}}$ dependent radius $R=\rho / p_{\mathrm{T}}$ [54], where the parameter $\rho$ is set to $30 \mathrm{GeV}$ and the radius is constrained to remain in the range between 0.02 and 0.4 [55]. The track jets are assigned to specific large- $R$ jets by ghost association with the original untrimmed large- $R$ jets. The main advantage of using such variable-radius track jets is that one can resolve the track jets from $H \rightarrow b \bar{b}$ decays at high $p_{\mathrm{T}}$ and retain the ability to double $b$-tag the large- $R$ Higgs-candidate jets.

Track jets are tagged as likely to contain $b$-hadrons if they satisfy the selection criteria of the MV2c10 algorithm $[56,57]$ that takes advantage of the relatively long lifetime and large mass of $b$-hadrons. A working point corresponding to a $b$-tagging efficiency of $77 \%$ for true $b$-jets is used. For this efficiency value, rejection factors of 5 and 110 are obtained against $c$-quark and light-quark jets, with the efficiency and rejection factors determined in $t \bar{t} \mathrm{MC}$ simulation [58].

Electrons are reconstructed by matching ID tracks to energy clusters in the EM calorimeter. The identification of electrons relies on a likelihood discriminant that takes the characteristic shape of electromagnetic showers into account [59]. Electrons are required to have $p_{\mathrm{T}}>7 \mathrm{GeV}$ and $|\eta|<2.47$, and satisfy the loose identification 
criteria [59]. The associated tracks must have a transverse impact parameter significance $\left|d_{0}\right| / \sigma_{d_{0}}<5$ relative to the beam axis and a longitudinal impact parameter $\left|z_{0} \sin \theta\right|<$ $0.5 \mathrm{~mm}$ relative to the primary vertex. Muons are reconstructed and identified by matching ID and MS tracks, and performing a global fit with all ID and MS measurements, taking the energy loss in the calorimeter into account [60]. Muons are required to have $p_{\mathrm{T}}>7 \mathrm{GeV}$ and $|\eta|<2.5$, and satisfy the loose identification criteria [60]. The following track requirements are applied: $\left|d_{0}\right| / \sigma_{d_{0}}<3$ and $\left|z_{0} \sin \theta\right|<0.5 \mathrm{~mm}$. Both the electrons and muons are required to satisfy loose isolation criteria $[59,60]$ based on the total transverse momentum of tracks surrounding the leptons within a cone of radius $\Delta R=\min \left(10 / p_{\mathrm{T}}^{\ell}[\mathrm{GeV}], \Delta R_{\max }\right)$, where $p_{\mathrm{T}}^{\ell}$ is the lepton $p_{\mathrm{T}}$, and $\Delta R_{\max }=0.2$ for electrons and 0.3 for muons. The isolation criteria have an efficiency of $99 \%$ with negligible dependence on the lepton $p_{\mathrm{T}}$ value.
Events must satisfy the trigger requirements and contain a primary vertex. In addition, noncollision backgrounds originating from calorimeter noise, beam halo interactions or cosmic rays are suppressed by rejecting events that contain any $R=0.4$ anti- $k_{t}$ calorimeter jet failing to satisfy a set of quality criteria. These are based on the LAr pulse shape, the energy profile of the jet in different parts of the calorimeter, and track variables [61]. Events with one or more charged leptons (electrons or muons) are also rejected to retain orthogonality with other $V H$ search channels.

The signal topology requires the presence of two large- $R$ jets with $|\eta|<2.0$ and $p_{\mathrm{T}}>200 \mathrm{GeV}$. The leading (highest $p_{\mathrm{T}}$ ) jet must satisfy $p_{\mathrm{T}}>500 \mathrm{GeV}$. The invariant mass of the dijet system consisting of the two highest- $p_{\mathrm{T}}$ jets in the event $\left(m_{J J}\right)$ is required to be larger than $1.3 \mathrm{TeV}$. These kinematic requirements guarantee that the trigger is fully efficient. To suppress $t$-channel multijet production, the difference between the rapidities of the two leading jets

TABLE I. Event selection requirements and definition of the different regions used in the analysis. Events in the signal, control, and validation regions must satisfy the preselection requirements.

\begin{tabular}{|c|c|}
\hline Preselection & $\begin{array}{l}\text { Veto non- } q q q q \text { channels: } \\
\text { No } e(\mu) \text { with } p_{\mathrm{T}}>7 \mathrm{GeV} \text { and }|\eta|<2.47(2.5) \\
\text { Event kinematics: } \\
\geq 2 \text { large- } R \text { jets with } p_{\mathrm{T}}>200 \mathrm{GeV} \text { and }|\eta|<2.0 \\
\text { leading large- } R \text { jet with } p_{\mathrm{T}}>500 \mathrm{GeV} \\
\text { leading and subleading large- } R \text { jets with } m_{J J}>1.3 \mathrm{TeV} \\
\text { leading and subleading large- } R \text { jets with }|\Delta y|<1.6\end{array}$ \\
\hline$V / H$ assignment & $V$-boson ( $H$-boson) candidate is large- $R$ jet with lower (higher) mass \\
\hline $\begin{array}{l}\text { Signal region } \\
(\mathrm{SRWH} / \mathrm{SRZH})\end{array}$ & $\begin{array}{l}V \text { and } H \text { bosons: } \\
W \text {-boson candidate within } W \text { jet mass, } D_{2} \text {, and } n_{\text {trk }} \text { windows } \\
Z \text {-boson candidate within } Z \text { jet mass, } D_{2} \text {, and } n_{\text {trk }} \text { windows } \\
H \text {-boson candidate within } H \text { jet mass, } n_{\text {trk }} \text { windows, with } 1 \text { or } 2 b \text {-tagged track jets }\end{array}$ \\
\hline $\begin{array}{l}\text { Control region } \\
(\mathrm{CR})\end{array}$ & $\begin{array}{l}\text { Fail both SRWH and SRZH } \\
\text { Pass } H \text {-boson candidate } n_{\text {trk }} \\
\left(m_{V}<65 \mathrm{GeV} \& m_{H}<70 \mathrm{GeV}\right) \text { or }\left(m_{V}>110 \mathrm{GeV} \& m_{H}>150 \mathrm{GeV}\right) \text { or } \\
\left(m_{V}<65 \mathrm{GeV} \& m_{H}>150 \mathrm{GeV}\right)\end{array}$ \\
\hline $\begin{array}{l}\text { Validation region } \\
\text { (VR1A) }\end{array}$ & $\begin{array}{l}\text { Fail both SRWH and SRZH } \\
\text { Pass } V \text {-boson candidate } n_{\text {trk }} \\
\text { Pass } H \text {-boson candidate } n_{\text {trk }} \\
65<m_{V}<110 \mathrm{GeV} \& m_{H}>150 \mathrm{GeV}\end{array}$ \\
\hline $\begin{array}{l}\text { Validation region } \\
\text { (VR1B) }\end{array}$ & $\begin{array}{l}\text { Fail both SRWH and SRZH } \\
\text { Fail } V \text {-boson candidate } n_{\text {trk }} \\
\text { Pass } H \text {-boson candidate } n_{\text {trk }} \\
65<m_{V}<110 \mathrm{GeV} \& m_{H}>150 \mathrm{GeV}\end{array}$ \\
\hline $\begin{array}{l}\text { Validation region } \\
\text { (VR2A) }\end{array}$ & $\begin{array}{l}\text { Fail both SRWH and SRZH } \\
\text { Pass } V \text {-boson candidate } n_{\text {trk }} \\
\text { Pass } H \text {-boson candidate } n_{\text {trk }} \\
m_{V}<65 \mathrm{GeV} \& 70<m_{H}<150 \mathrm{GeV}\end{array}$ \\
\hline $\begin{array}{l}\text { Validation region } \\
\text { (VR2B) }\end{array}$ & $\begin{array}{l}\text { Fail both SRWH and SRZH } \\
\text { Fail } V \text {-boson candidate } n_{\text {trk }} \\
\text { Pass } H \text {-boson candidate } n_{\text {trk }} \\
m_{V}<65 \mathrm{GeV} \& 70<m_{H}<150 \mathrm{GeV}\end{array}$ \\
\hline
\end{tabular}


must satisfy $|\Delta y|<1.6$. Only the two leading- $p_{\mathrm{T}}$ jets are retained for further consideration.

As an initial step, the jet with the larger mass is taken to be the $H$-boson candidate while the other jet is taken to be the $V$-boson candidate. Discrimination between these $V$-boson or $H$-boson candidates and background jets relies on several properties of the large- $R$ jets: mass, $D_{2}$, and $n_{\text {trk }}$. An optimization procedure is applied to adjust the selection criteria involving those variables to maximize the significance of the resonance signal under study. In the case of $V$-boson jets, the selection is based on the three discriminating variables as developed in the search for heavy diboson resonances in the fully hadronic channel [47], with the exception that the $n_{\text {trk }}$ selection is loosened slightly. In the case of the $H$-boson candidates, the selection criteria are optimized with regard to the jet mass and $n_{\text {trk }}$. The successful $\mathrm{H}$-boson candidate has at least one associated track jet and can be classified as either 1-tag or 2-tag, depending on the number of track jets satisfying the $b$-tagging requirements. Only the two highest- $p_{\mathrm{T}}$ associated track jets are considered for $b$-tagging. The variable $D_{2}$ provides little additional discrimination and is thus dropped.

The $p_{\mathrm{T}^{-}}$-dependent jet mass windows for the $W(Z)$-boson candidates that result from the optimization procedure vary from 80-100 (85-110) $\mathrm{GeV}$ for jets with $p_{\mathrm{T}}$ around $500 \mathrm{GeV}$ to $55-130(65-135) \mathrm{GeV}$ for jets with $p_{\mathrm{T}}$ around $3000 \mathrm{GeV}$. The upper bounds on $D_{2}$ for the $V$-boson candidates vary approximately from 1.0 to 1.5 , for the same $p_{\mathrm{T}}$ values. The upper cut on the $n_{\text {trk }}$ variable varies between 25 (26) and 31 (29) for $W(Z)$-boson candidates, with looser requirements for higher- $p_{\mathrm{T}}$ jets. Given the experimental jet mass resolution, no exclusive selection of $W$ or $Z$ bosons is performed and the $W H$ and $Z H$ final states are searched for independently. For $H$-boson candidates, the mass windows applied to events in the 1-tag (2-tag) category vary from 80-135 (95-150) GeV to 105-155 (100-170) GeV for jets with $p_{\mathrm{T}}$ of 500 and $3000 \mathrm{GeV}$, respectively. The $H$-boson candidates classified as 1(2)-tag are required to have an associated $n_{\text {trk }}$ value below 32 (35) to 44 (55), looser at higher $p_{\mathrm{T}}$.

The event selection defining the signal region (SR) is summarized in Table I. The resulting signal acceptance times efficiency $(\mathcal{A} \times \varepsilon)$ for events in each category is shown in Figure 2. In the 1-tag category, $\mathcal{A} \times \varepsilon$ rises from approximately $3 \%$ to $10 \%$ for $W H$ resonance masses increasing from 1.5 to $5.0 \mathrm{TeV}$. In the 2-tag category, $\mathcal{A} \times \varepsilon$ remains essentially constant at $4 \%$ for $W H$ resonances. The different trends for the 1-tag and 2-tag selections as a function of $m\left(V^{\prime}\right)$ are the result of a combination of effects including the $p_{\mathrm{T}}$ dependence of both $V$ - and $H$-tagging as well as the signal to background ratio. The $\mathcal{A} \times \mathcal{E}$ values are about $0.5 \%$ lower for $Z H$ resonances due to the smaller mass separation between $Z$ and $H$ bosons. The experimental mass resolution for resonance masses of 2 (4) $\mathrm{TeV}$ is $3.5 \%(2.6 \%)$.

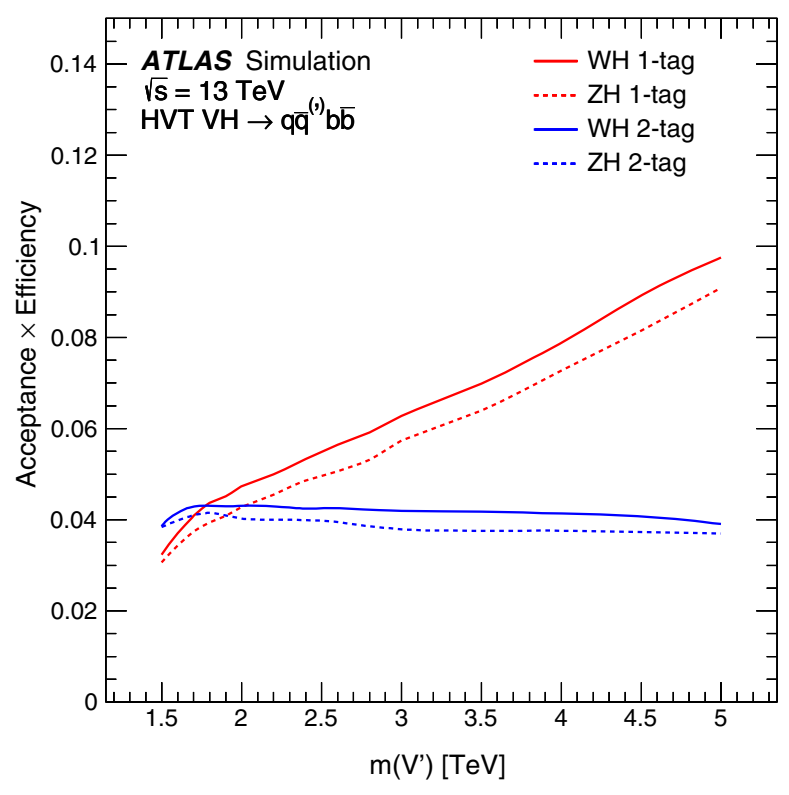

FIG. 2. Signal acceptance times efficiency as a function of the resonance mass, for events in the $W H$ (solid lines) and $Z H$ (dashed lines) signal regions, in the 1-tag and 2-tag categories, with respect to the total number of generated events in each sample. The HVT MC samples include only $V^{\prime} \rightarrow V H$ decays with $V \rightarrow q \bar{q}^{(\prime)}$ and $H \rightarrow b \bar{b}$ or $c \bar{c}$.

\section{BACKGROUND ESTIMATION AND EVENT YIELDS}

The dominant background in this search comes from multijet events, corresponding to at least $85 \%$ of the total background in the signal regions, where the remaining events come from $t \bar{t}$ and $V+$ jets processes.

The totality of the background is estimated via a datadriven method that provides template $m_{J J}$ distributions for the $W H$ and $\mathrm{ZH}$ final states in the 1-tag and 2-tag categories. These background $m_{J J}$ templates are obtained in three steps: (i) background templates for the $\mathrm{WH}$ and $\mathrm{ZH}$ final states are extracted from data in the 0-tag category, where the $H$-boson candidate has zero associated $b$-tagged jets; (ii) yield and shape corrections are derived from a control region and applied to these templates; and (iii) a rebinning and smoothing of the resulting $m_{J J}$ distributions is performed, to ensure robustness against statistical fluctuations. All steps are described in this section.

To define the 0-tag templates from which the background in the 1-tag and 2-tag categories is extracted, additional requirements are placed on 0-tag events, such that at least 1(2) variable-radius track jet(s) is (are) associated with the $H$-boson jet, when estimating the background in the 1(2)tag category. Events in the 0 -tag category are not expected to directly describe either the yield or the $m_{J J}$ shape of the background in the 1-tag and 2-tag categories without further corrections: the $b$-tagging requirements enhance heavy-flavor components in the background and introduce kinematics-dependent effects. 
Therefore, a control region (CR) is used to estimate the yield and shape corrections to the $m_{J J}$ distribution needed to extrapolate the 0-tag background events to the 1(2)-tag SRs. The CR has negligible contamination from signal. Validation regions (VR) with events that fail the SR selection are used to confirm the effectiveness of the background model and derive the associated systematic uncertainties. The definitions of the control and validation regions are shown in detail in Table I and illustrated in Fig. 3. According to simulation, multijet processes are responsible for $85 \%$ to $99 \%$ of the background composition across the different control, validation, and signal regions. This variation and its impact on the background estimate are taken into account by the uncertainties described in Sec. VI.

To define the background normalization in the 1(2)-tag category, a normalization correction $\mu_{\mathrm{CR}}^{1(2) \text {-tag }}$ is extracted from the ratio of 1(2)-tag to 0 -tag yields in the control region:

$$
\mu_{\mathrm{CR}}^{1(2)-\operatorname{tag}}=\frac{N_{\mathrm{CR}}^{1(2)-\operatorname{tag}}}{N_{\mathrm{CR}}^{0-\operatorname{tag}}},
$$

where $N_{\mathrm{CR}}^{0 \text {-tag }}$ and $N_{\mathrm{CR}}^{1(2) \text {-tag }}$ are the numbers of events observed in the 0-tag and 1(2)-tag CR event categories. The values of $\mu_{\mathrm{CR}}^{1-\operatorname{tag}}$ and $\mu_{\mathrm{CR}}^{2-\operatorname{tag}}$ are determined to be $0.160 \pm$ 0.014 and $0.0167 \pm 0.0028$, respectively, where the uncertainties are dominated by systematic effects discussed in Sec. VI. The difference in the corrections for 1-tag and 2-tag events can be understood based on studies of simulated multijet events. In 1-tag events, the two leading track jets associated with Higgs candidate large- $R$ jets are dominated by one true $b$-jet accompanied by one true lightjet, whereas in 2-tag events they are dominated by two true $b$-jets. This indicates that different processes are at work in

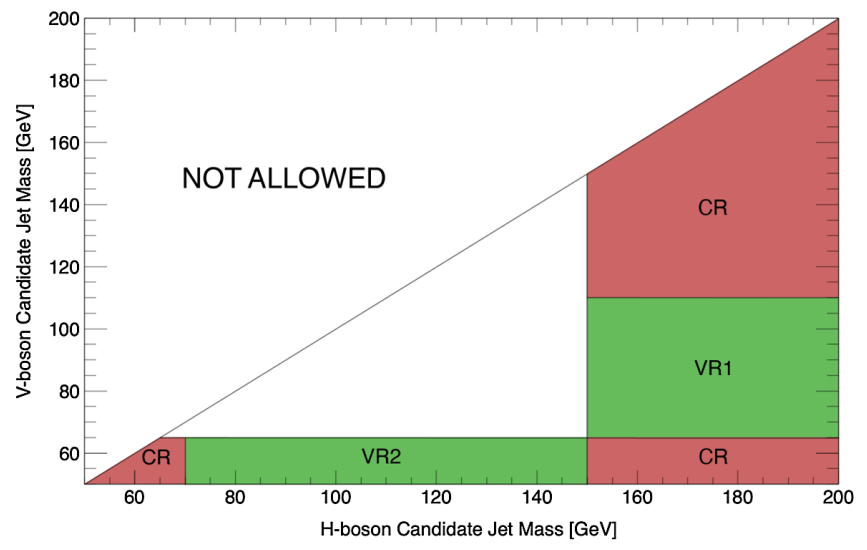

FIG. 3. Illustration of control and validation regions, defined by the masses of the $H$-boson and $V$-boson candidates. The regions VR1 and VR2 are further split into two regions each, according to the $n_{\text {trk }}$ requirement on the $V$-boson candidate. The same definitions are applied across the number of $b$-tags (0-, 1-, and 2-tag categories). 1-tag and 2-tag events. The expected number of background 1(2)-tag events in the SR is calculated by applying the $\mu_{\mathrm{CR}}^{1(2) \text {-tag }}$ correction factor to the number of 0 -tag events that pass all SR requirements except for the $b$-tagging.

To extract $m_{J J}$ background templates from the 0 -tag category, a multidimensional kinematic reweighting [62] is performed using the control region events. A boosted decision tree (BDT) is used to perform the reweighting, by predicting the event weights needed to bring the shapes of kinematic distributions in the 0 -tag and 1(2)-tag categories into agreement. The training is performed in the CR data and performed separately for 1-tag and 2-tag events. Variables that are sensitive to the presence of $b$-jets associated with the $H$-boson candidate and to the resulting kinematic differences are used to train the BDTs: the fourmomenta of the two leading variable-radius track jets and their angular separation, the transverse momenta of the $H$ - and $V$-boson jets, and the number of tracks and variableradius track jets associated with the $H$-boson jet. The BDTs are built with 100 trees, a maximum depth of three layers, and a minimum of 500 events per leaf, with a learning rate set to 0.1 .

In order to quantify the effectiveness of the reweighting, a binary classifier was trained to differentiate between the reweighted and target $m_{J J}$ distributions in the validation regions, and observed to classify them correctly at most $53 \%$ of the time, consistent with random guessing. The observed distributions of kinematic variables, including $m_{J J}$, are found to be well described by the background model for 1-tag and 2-tag events in VR1A, VR1B, VR2A, and VR2B (defined in Table I).

The modeling of the $m_{J J}$ distributions in the VR2B region is shown in Fig. 4, for 1-tag and 2-tag events. A residual disagreement between the data and the expected background after reweighting is accounted for by a systematic uncertainty, as discussed in Sec. VI.

The numbers of 1-tag and 2-tag events observed in the control and validation regions are shown in Table II and compared with the predicted background yields.

After the normalization and reweighting corrections are applied to the events in the 0-tag category, the expected $m_{J J}$ background distributions in 1-tag and 2-tag categories are produced with a variable bin width that reflects the experimental mass resolution. Those distributions are then fit using a functional form that captures the smoothly falling behavior of the background:

$$
f_{\text {Background }}(x)=\mathrm{e}^{-p_{0}}(1-x)^{-p_{1}} x^{-p_{2}},
$$

where $x=m_{J J} / \sqrt{s}$ and $p_{0}, p_{1}$, and $p_{2}$ are the fit parameters. The results of these fits provide the background estimates that are used in the statistical analysis (described in Sec. VII) for the different signal regions. 

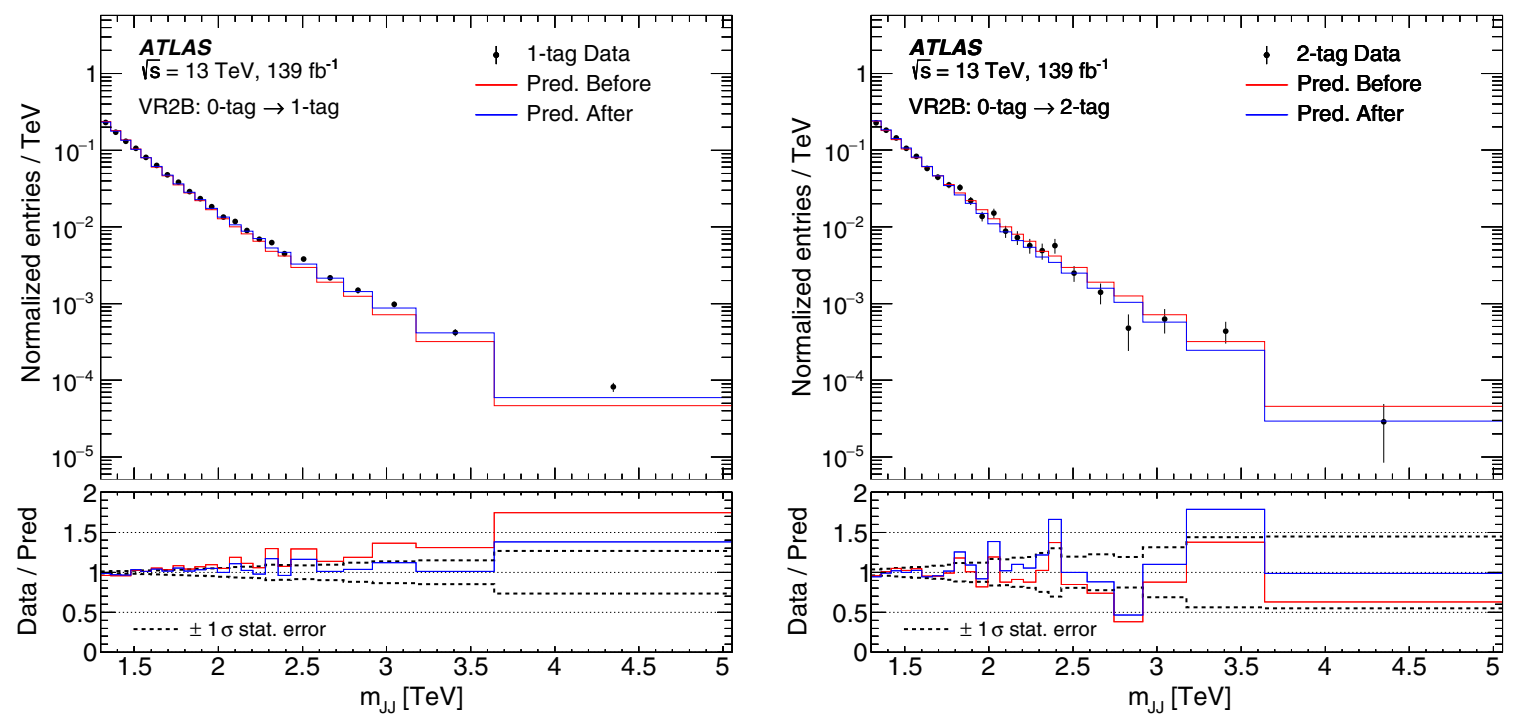

FIG. 4. Dijet mass distributions in the 1-tag (left) and 2-tag (right) VR2B regions compared with the predicted background extracted from the 0 -tag events (histograms) before and after BDT reweighting.

\section{SYSTEMATIC UNCERTAINTIES}

Systematic uncertainties arise from several different sources: the data-driven background estimate, the modeling of experimental uncertainties affecting the signal, and the impact of signal theory uncertainties. At a resonance mass of $2 \mathrm{TeV}$, the background normalization and shape uncertainties dominate, while at a resonance mass of $4 \mathrm{TeV}$, the large-radius jet and boson tagging uncertainties dominate due to the small background contribution at high $m_{J J}$.

The uncertainties affecting both the normalization and shape of the background predictions are determined from the validation regions. These uncertainties arise from limited sample sizes and from differences in the background

TABLE II. Data and estimated background yields for 1-tag and 2 -tag events in the control and validation regions. The uncertainties correspond to the combination of statistical and systematic components. By construction, the uncertainties cover the differences between the observed and expected yields in the validation regions.

\begin{tabular}{lrc}
\hline \hline 1-tag & Data & Background prediction \\
\hline Control region (CR) & 48668 & -- \\
Validation region (VR1A) & 4440 & $4290 \pm 380$ \\
Validation region (VR1B) & 18361 & $18000 \pm 1600$ \\
Validation region (VR2A) & 11844 & $12600 \pm 1100$ \\
Validation region (VR2B) & 29436 & $31200 \pm 2700$ \\
\hline 2-tag & Data & Background prediction \\
\hline Control region (CR) & 4976 & -- \\
Validation region (VR1A) & 507 & $443 \pm 74$ \\
Validation region (VR1B) & 1922 & $1860 \pm 310$ \\
Validation region (VR2A) & 1337 & $1290 \pm 220$ \\
Validation region (VR2B) & 3065 & $3200 \pm 540$ \\
\hline \hline
\end{tabular}

composition in the various regions. The normalization uncertainty is taken to be the difference between the smallest and largest $\mu^{1(2) \text {-tag }}$ values obtained in any of the validations regions, resulting in a systematic error of $9 \%(17 \%)$ in the background estimate for the 1(2)-tag category. The uncertainty in the shape of the background $m_{J J}$ distribution is assessed from the ratio of data to prediction in the VR2B region, where the differences are the largest. This uncertainty is determined after smoothing the $m_{J J}$ distributions for both the data and the background prediction with the same functional form as described in Sec. V. It results in changes to the background yield of approximately $5 \%$ at $m_{J J}$ of $2 \mathrm{TeV}$ and up to $24 \%$ at $4 \mathrm{TeV}$.

An additional shape uncertainty is assigned to account for the choice of fitting function, assessed by fitting alternate empirical functions, amounting to a maximum uncertainty of $2 \%(14 \%)$ at an $m_{J J}$ value of 2 (4) TeV.

Experimental uncertainties related to MC simulation are applicable only to signal samples, and are divided into two categories: $b$-tagging and large- $R$ jets. A set of $b$-tagging correction factors and corresponding uncertainties are applied as a function of $p_{\mathrm{T}}$ and $\eta$ of the variable-radius track jets to match the efficiencies for tagging $b$-jets measured in data, determined with $t \bar{t}$ events [58]. The uncertainties in the correction factors are extrapolated for track jets with $p_{\mathrm{T}}$ larger than $400 \mathrm{GeV}$. An additional extrapolation uncertainty is obtained by varying the inputs to the $b$-tagging algorithm according to their modeling uncertainties and by recomputing its efficiency in MC simulation [58].

Uncertainties in the $p_{\mathrm{T}}$ and mass scales of the large- $R$ jet are determined with the $R_{\text {trk }}$ method [63] adapted to the jet collection used in this article, relying on independent measurements by the calorimeter and the inner detector, 
TABLE III. Systematic uncertainties affecting the signal event yields in the $W H$ and $Z H$ signal regions with 1-tag or 2-tag (denoted WH-1, WH-2, ZH-1, and ZH-2, respectively). The HVT model is used with resonance masses of 2 and $4 \mathrm{TeV}$.

\begin{tabular}{|c|c|c|c|c|c|c|c|c|}
\hline \multirow[b]{2}{*}{ Source } & \multicolumn{4}{|c|}{ Signal (2 TeV) } & \multicolumn{4}{|c|}{ Signal (4 TeV) } \\
\hline & WH-1 & WH-2 & $\mathrm{ZH}-1$ & $\mathrm{ZH}-2$ & WH-1 & WH-2 & $\mathrm{ZH}-1$ & $\mathrm{ZH}-2$ \\
\hline Jet energy scale & $2.8 \%$ & $3.1 \%$ & $1.6 \%$ & $2.1 \%$ & $5.2 \%$ & $8.4 \%$ & $6.6 \%$ & $8.0 \%$ \\
\hline Jet mass scale & $18 \%$ & $18 \%$ & $9.6 \%$ & $14 \%$ & $20 \%$ & $17 \%$ & $21 \%$ & $18 \%$ \\
\hline Jet mass resolution & $43 \%$ & $45 \%$ & $37 \%$ & $40 \%$ & $22 \%$ & $21 \%$ & $21 \%$ & $22 \%$ \\
\hline Flavor tagging & $17 \%$ & $8.4 \%$ & $16 \%$ & $8.4 \%$ & $26 \%$ & $12 \%$ & $26 \%$ & $13 \%$ \\
\hline$H$-boson tagging & $10 \%$ & $10 \%$ & $10 \%$ & $10 \%$ & $10 \%$ & $10 \%$ & $10 \%$ & $10 \%$ \\
\hline$V$-boson tagging & $13 \%$ & $13 \%$ & $13 \%$ & $13 \%$ & $13 \%$ & $13 \%$ & $13 \%$ & $13 \%$ \\
\hline Luminosity & $1.7 \%$ & $1.7 \%$ & $1.7 \%$ & $1.7 \%$ & $1.7 \%$ & $1.7 \%$ & $1.7 \%$ & $1.7 \%$ \\
\hline MC statistical uncertainty & $1.5 \%$ & $1.4 \%$ & $1.6 \%$ & $1.4 \%$ & $1.2 \%$ & $1.4 \%$ & $1.5 \%$ & $1.7 \%$ \\
\hline
\end{tabular}

and are of the order of 5\% to $10 \%$ each. These uncertainties lead to shifts in $m_{J J}$ of the resonant signal peak as well as differences in the signal selection efficiency. The effects of resolution uncertainties on the $p_{\mathrm{T}}$ and mass measurements are estimated by degrading the resolution in MC simulation according to a Gaussian smearing of width 0.02 in $\sigma\left(p_{\mathrm{T}}\right) / p_{\mathrm{T}}$ and $0.20 \times \sigma(m) / m$ in $\sigma(m) / m[64,65]$.

The $H$ - and $V$-boson tagging techniques are assigned dedicated uncertainties to take into account the requirements on $D_{2}$ and $n_{\text {trk }}$. An MC efficiency correction factor of $0.92 \pm 0.13$ for $V$-boson tagging was determined in the search for heavy diboson resonances in the fully hadronic channel [47] by taking advantage of a control region in data that is enhanced in $V+$ jets events. Given that the same method for $V$-tagging is used in this analysis, the same scale factor and uncertainty of $14 \%$ is assigned to the signal normalization. ${ }^{3}$ An efficiency correction and uncertainty in the $n_{\text {trk }}$ requirement was also estimated in Ref. [66] for $V$ jets. This uncertainty is applied to $H$-tagging, with an additional component to cover topology differences, based on simulation studies, corresponding to a total $10 \%$ uncertainty. In particular, these studies compare the largeradius jet mass distributions between the data and the simulation, and the impact of the $n_{\text {trk }}$ requirement on the data-to-MC agreement in the $V$-mass and $H$-mass regions.

Signal cross sections computed at leading order are used in the interpretation of the results. The impact of uncertainties in the PDF sets, initial- and final-state radiation, and multiparton interactions on the signal acceptance are included. Uncertainties related to the PDF sets are derived by applying the methodology outlined by the PDF4LHC group [24] and considering four additional PDF sets (CT14, MMHT2014, NNPDF3.0, and ATLAS-epWZ12), resulting in $<1 \%$ uncertainties in the signal acceptance. An uncertainty due to choosing the A14 tune for the signal

\footnotetext{
${ }^{3}$ The only difference is in the $n_{\text {trk }}$ requirement, which is looser in this analysis. Studies of relative signal efficiencies for the different $n_{\text {trk }}$ requirements show that this approximation is well motivated.
}

generation is estimated by varying the scales for initial- and final-state radiation, as well as multiparton interactions, and results in an uncertainty of $2 \%(3 \%)$ for $W H(\mathrm{ZH})$ resonances.

Finally, an uncertainty in the Run 2 integrated luminosity of $1.7 \%$ [22] is considered, as obtained by the LUCID-2 detector [67] for the main luminosity measurements. The impact of the main systematic uncertainties on signal event yields is summarized in Table III.

\section{RESULTS}

The statistical analysis of the data is performed using a binned likelihood function, constructed from the $m_{J J}$ distributions in the 1-tag and 2-tag signal regions, using the procedure described in Ref. [1] and the ROOSTATS framework [68]. The $m_{J J}$ histograms derived from MC simulation are used for the HVT $W^{\prime}$ and $Z^{\prime}$ processes, while the data-driven background estimates are used for the combined $t \bar{t}, V+$ jets, and QCD multijet processes. The input $m_{J J}$ distribution bounds are $[1.3,6.0] \mathrm{TeV}$.

A test statistic based on the profile likelihood ratio [69] is used to test signal hypotheses, parametrized by the signal strength value, $\mu$, acting as a scale factor on the predicted number of signal events for each model assumption. The likelihood, $L$, is defined from the Poisson probability to observe $N$ data events for a given signal $s$ and background $b$ expectation in each bin of the final discriminant:

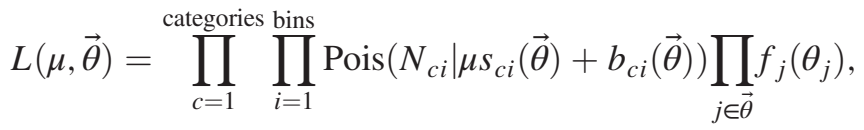

where the index $c$ represents the 1-tag or 2-tag event categories and $i$ represents the bin in the $m_{J J}$ distribution. Nuisance parameters $\vec{\theta}$ are included in the likelihood function with Gaussian or log-normal constraint terms, $f_{j}\left(\theta_{j}\right)$. Those nuisance parameters which produce bin variations smaller than $1 \%$ from the nominal value are neglected. 
Experimental uncertainties in the signal are fully correlated between the 1-tag and 2-tag signal regions, whereas background modeling uncertainties are kept independent. In order to avoid an overconstraining of the background modeling uncertainties in the high mass region due to the higher statistical power at low masses, the $m_{J J}$ shape uncertainties above and below $2.5 \mathrm{TeV}$ are allowed to vary independently in the fit. The postfit background expectation was found to be stable and independent of the particular choice of splitting point.

The fits are performed separately for the $W^{\prime}$ and $Z^{\prime}$ models, using data in the 1-tag and 2-tag regions from the
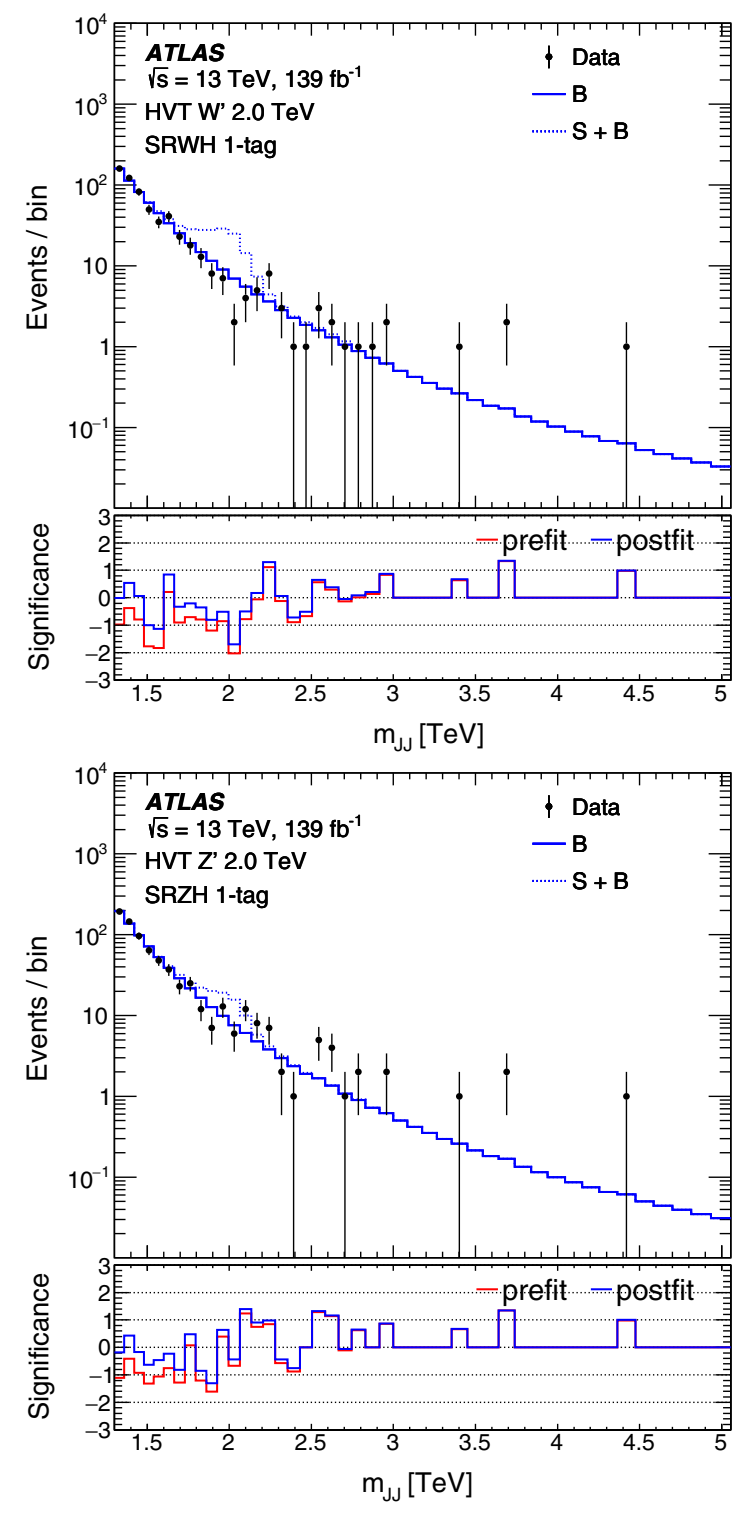

SRWH and SRZH selections, respectively. The fit results are interpreted independently for the $W^{\prime}$ and $Z^{\prime}$ hypotheses - the $\mathrm{WH}$ and $\mathrm{ZH}$ signal regions are not orthogonal and have approximately $40 \%$ of events in common, in each category.

The pre- and postfit $m_{J J}$ distributions in the signal region are shown in Fig. 5 for signal resonances with a mass of $2 \mathrm{TeV}$. The numbers of data events in the signal regions are shown in Table IV, along with the predicted background and signal yields, postfit. No events with $m_{J J}$ values above $5 \mathrm{TeV}$ are selected.

A test of the background-only hypothesis is performed by setting $\mu$ equal to zero in the likelihood fit. Deviations
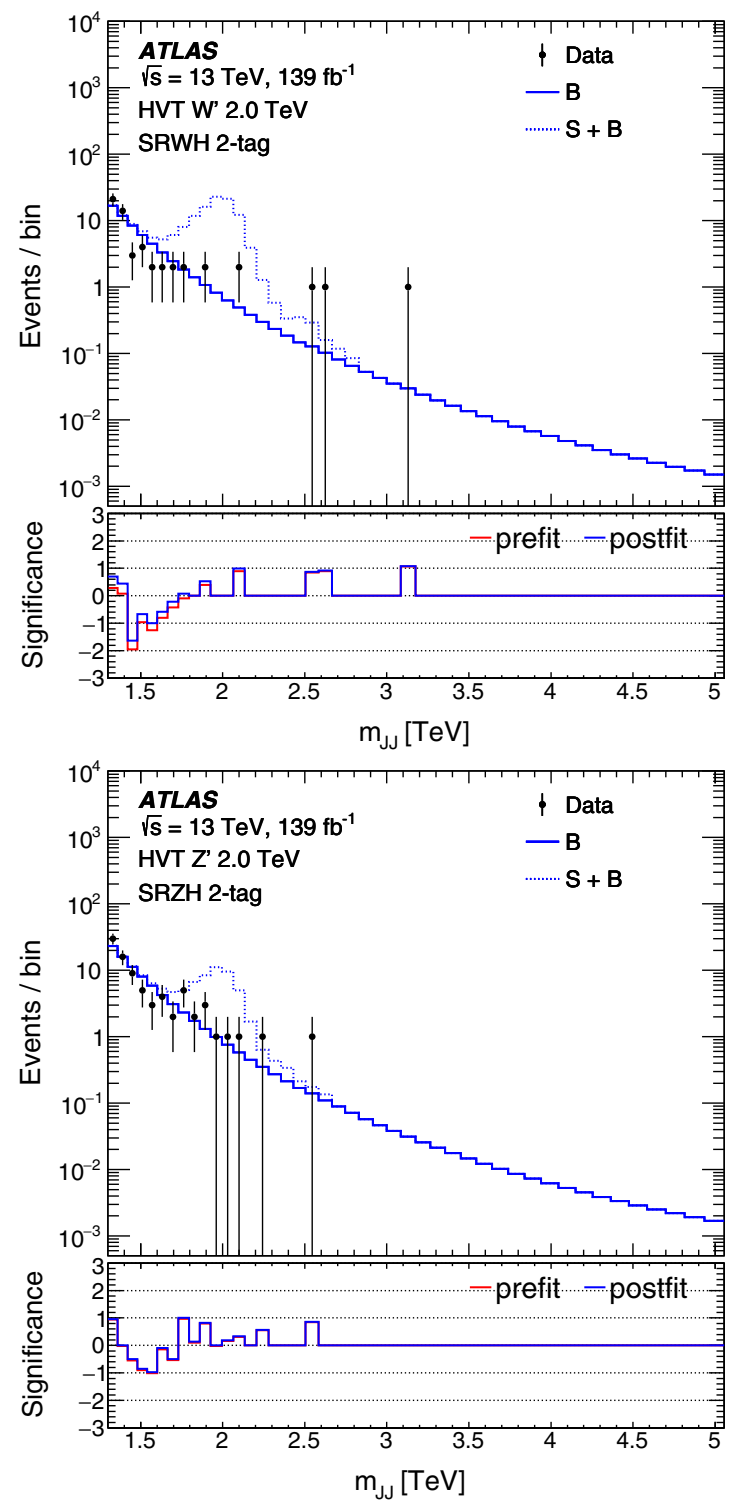

FIG. 5. Dijet mass distributions in the $W H$ (top) and $Z H$ (bottom) signal regions, after the likelihood fit to events in the 1-tag (left) and 2-tag (right) categories. The black points correspond to data and the solid blue histogram to the postfit background prediction. The $W H$ and $\mathrm{ZH}$ signal regions are not orthogonal. The expected signal distributions for a $V^{\prime}$ boson with mass of $2 \mathrm{TeV}$ are also shown (dashed histograms). The bin width varies and corresponds to the experimental mass resolution. Distributions of the significance of the observed deviations from the expected background are presented in the bottom panels before and after the fit. The significance calculation assumes Poisson probabilities and only accounts for statistical fluctuations. 
TABLE IV. Event yields for the data, predicted background, and signal in each of the signal regions. The signal corresponds to that expected for HVT model B with resonance masses of 2 and $4 \mathrm{TeV}$.

\begin{tabular}{lrccc}
\hline \hline Region & Data & Background & $\begin{array}{c}\text { Signal } \\
(2 \mathrm{TeV})\end{array}$ & $\begin{array}{c}\text { Signal } \\
(4 \mathrm{TeV})\end{array}$ \\
\hline SRWH 1-tag & 598 & $612 \pm 46$ & 110 & 2.0 \\
SRWH 2-tag & 57 & $61 \pm 13$ & 100 & 1.0 \\
SRZH 1-tag & 717 & $725 \pm 53$ & 47 & 0.80 \\
SRZH 2-tag & 84 & $81 \pm 17$ & 44 & 0.42 \\
\hline \hline
\end{tabular}

from the background-only hypothesis are quantified by determining the local $p_{0}$-value at each signal mass point. The largest deviation is observed in the fit to the $W H$ signal regions and corresponds to a $p_{0}$-value of 0.03 for a resonance mass of $2.8 \mathrm{TeV}$.

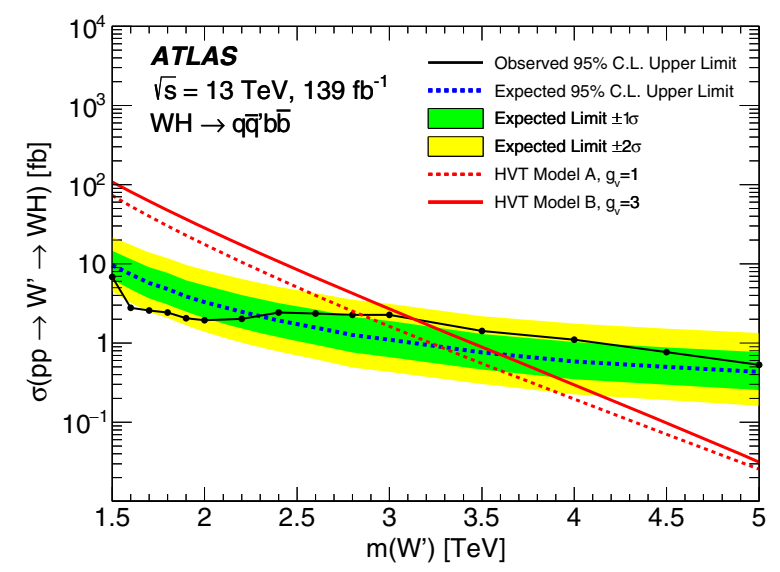

The data are used to set upper limits on the production cross section of new resonances. Exclusion limits are computed using the CLs method [70], with a value of $\mu$ regarded as excluded at the 95\% C.L. when the CLs value is less than 5\%. The cross-section limits are shown in Fig. 6. The observed limits range from cross sections of 6.8 to $0.53 \mathrm{fb}$ for $W H$ and from 8.7 to $0.53 \mathrm{fb}$ for $Z H$, corresponding to the exclusion of $W^{\prime}\left(Z^{\prime}\right)$ resonances up to a mass of $2.90 \mathrm{TeV}(2.20 \mathrm{TeV})$ in the context of HVT model A and $3.20 \mathrm{TeV}(2.65 \mathrm{TeV})$ in the context of HVT model B. The 2-tag category dominates the sensitivity of the search at low resonance mass while the impact of the 1-tag category increases at higher mass, surpassing the 2-tag category at masses above $3.9 \mathrm{TeV}$. As a test of the asymptotic approximation used in the statistical analysis, results are also obtained with ensembles of

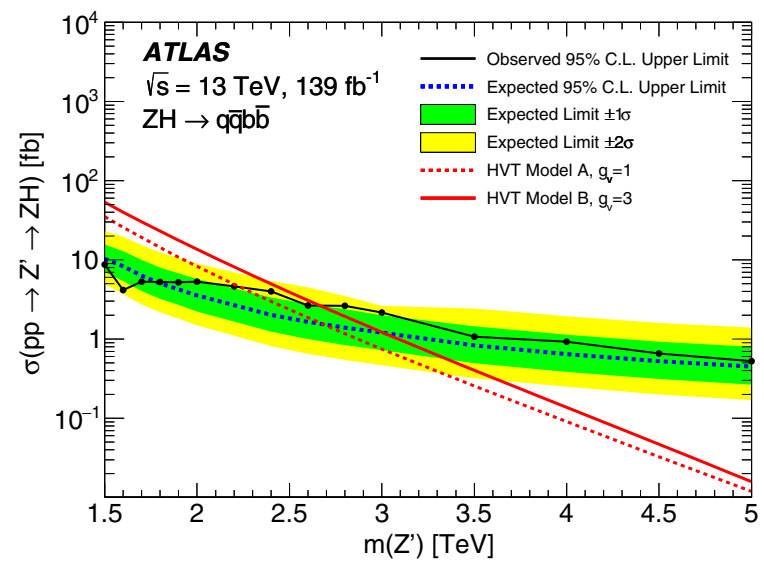

FIG. 6. Observed and expected 95\% C.L. upper limits on the cross section for $p p \rightarrow V^{\prime} \rightarrow V H$ in the $W H$ (left) and $Z H$ (right) channels. The red solid (dashed) lines show the cross-section predictions as a function of the resonance mass in the context of HVT model B (A).
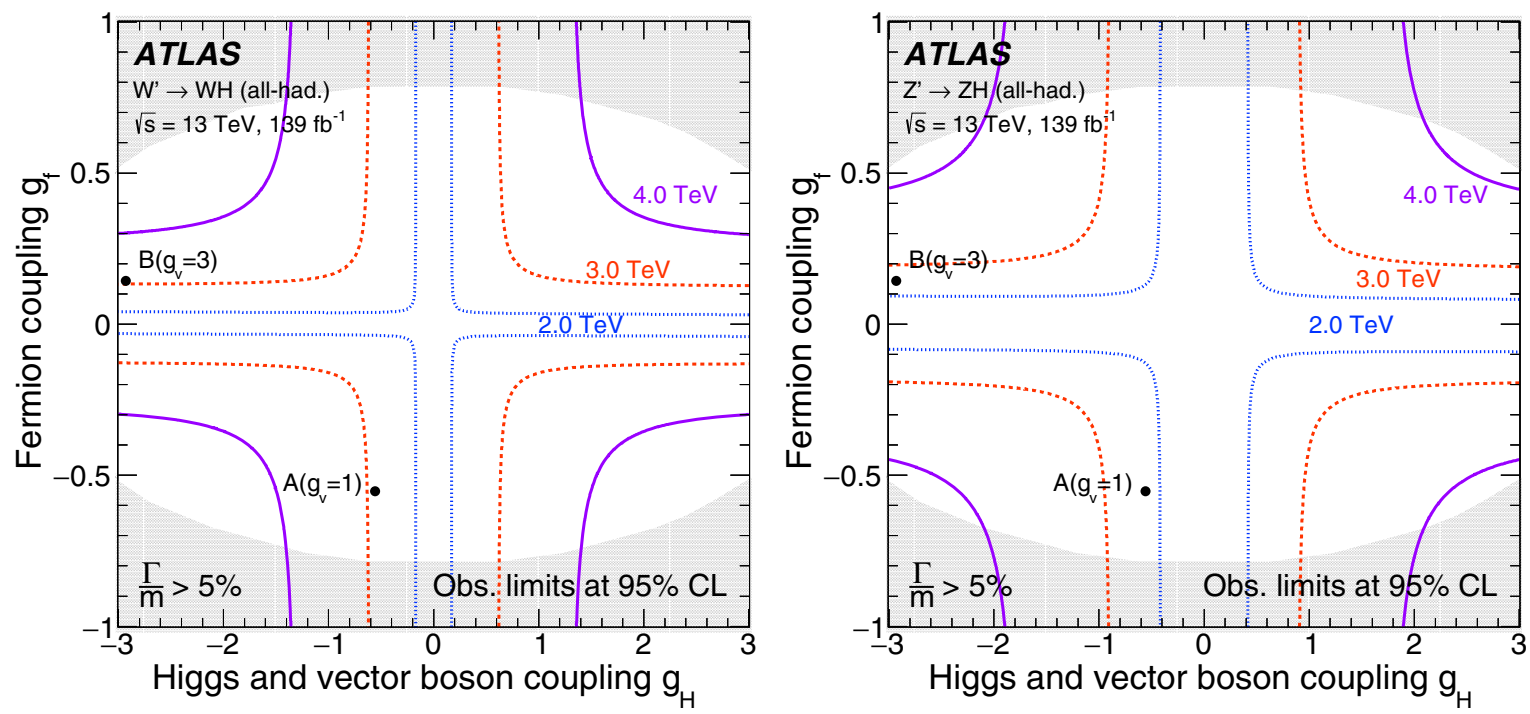

FIG. 7. Limits at $95 \%$ C.L. in the $g_{f}$ vs $g_{H}$ plane for resonance masses of 2, 3, and $4 \mathrm{TeV}$ for the $W H$ (left) and $Z H$ (right) channels in the context of the HVT model. The coupling values corresponding to HVT models A and B are indicated by filled circles. The gray region indicates values of the couplings corresponding to $V^{\prime}$ resonances with $\Gamma / m$ greater than $5 \%$. In that region, the assumption that the $V^{\prime}$ signal $m_{J J}$ shape is dominated by the experimental resolution is no longer valid. 
pseudoexperiments. The cross-section upper limits obtained in that case are looser by 10-20\%, with a larger difference at high $m\left(V^{\prime}\right)$, and the mass limits are at most $0.05 \mathrm{TeV}$ weaker.

These results can also be translated into exclusions in the $\left\{g_{H}, g_{f}\right\}$ plane, where $g_{f}$ represents a universal coupling between the $V^{\prime}$ bosons and fermions. Here, $g_{q}$ is taken to be equal to $g_{f}$. Figure 7 shows the $95 \%$ C.L. limits in this plane for several resonance masses.

\section{CONCLUSION}

A search for heavy resonances decaying into a $W$ or $Z$ boson and a Higgs boson is reported. The results are based on a sample of $p p$ collisions at $\sqrt{s}=13 \mathrm{TeV}$ collected by the ATLAS detector at the Large Hadron Collider, corresponding to $139 \mathrm{fb}^{-1}$ of integrated luminosity. The search exploits jet substructure techniques to study the fully hadronic $q q b b$ final state which results from the dominant decay modes of the $W / Z$ and Higgs bosons. The main background contribution arises from multijet production, with a smaller contribution from top-quark pair and $V+$ jets production. All background contributions are extracted directly from the data. Compared with the previously available results, the search benefits from an increased integrated luminosity, as well as from improvements in reconstruction and tagging of large- $R$ jets and track jets with $p_{\mathrm{T}}$-dependent radius.

No significant excess of events is observed over the expected background and the upper limits set on the cross section for $p p \rightarrow W^{\prime} \rightarrow W H$ and $p p \rightarrow Z^{\prime} \rightarrow Z H$ range from $6.8 \mathrm{fb}$ at $m\left(W^{\prime}\right)=1.5 \mathrm{TeV}$ to $0.53 \mathrm{fb}$ at $m\left(W^{\prime}\right)=5.0 \mathrm{TeV}$, and from $8.7 \mathrm{fb}$ at $m\left(Z^{\prime}\right)=1.5 \mathrm{TeV}$ to $0.53 \mathrm{fb}$ at $m\left(Z^{\prime}\right)=5.0 \mathrm{TeV}$, at $95 \%$ C.L. These results translate into lower limits on the mass of $W^{\prime}\left(Z^{\prime}\right)$ bosons of $2.90 \mathrm{TeV}(2.20 \mathrm{TeV})$ in the context of the weakly coupled HVT model A and of $3.20 \mathrm{TeV}(2.65 \mathrm{TeV})$ in the context of the strongly coupled HVT model B, at 95\% C.L.

\section{ACKNOWLEDGMENTS}

We thank CERN for the very successful operation of the LHC, as well as the support staff from our institutions without whom ATLAS could not be operated efficiently.
We acknowledge the support of ANPCyT, Argentina; YerPhI, Armenia; ARC, Australia; BMWFW and FWF, Austria; ANAS, Azerbaijan; SSTC, Belarus; CNPq and FAPESP, Brazil; NSERC, NRC, and CFI, Canada; CERN; ANID, Chile; CAS, MOST, and NSFC, China; COLCIENCIAS, Colombia; MSMT CR, MPO CR, and VSC CR, Czech Republic; DNRF and DNSRC, Denmark; IN2P3-CNRS and CEA-DRF/IRFU, France; SRNSFG, Georgia; BMBF, HGF, and MPG, Germany; GSRT, Greece; RGC and Hong Kong SAR, China; ISF and Benoziyo Center, Israel; INFN, Italy; MEXT and JSPS, Japan; CNRST, Morocco; NWO, Netherlands; RCN, Norway; MNiSW and NCN, Poland; FCT, Portugal; MNE/IFA, Romania; MES of Russia and NRC KI, Russia Federation; JINR; MESTD, Serbia; MSSR, Slovakia; ARRS and MIZŠ, Slovenia; DST/NRF, South Africa; MICINN, Spain; SRC and Wallenberg Foundation, Sweden; SERI, SNSF and Cantons of Bern and Geneva, Switzerland; MOST, Taiwan; TAEK, Turkey; STFC, United Kingdom; DOE and NSF, United States of America. In addition, individual groups and members have received support from BCKDF, CANARIE, Compute Canada and CRC, Canada; ERC, ERDF, Horizon 2020, Marie Skłodowska-Curie Actions and COST, European Union; Investissements d'Avenir Labex, Investissements d'Avenir Idex and ANR, France; DFG and AvH Foundation, Germany; Herakleitos, Thales, and Aristeia programmes cofinanced by EU-ESF and the Greek NSRF, Greece; BSF-NSF and GIF, Israel; La Caixa Banking Foundation, CERCA Programme Generalitat de Catalunya and PROMETEO and GenT Programmes Generalitat Valenciana, Spain; Göran Gustafssons Stiftelse, Sweden; The Royal Society and Leverhulme Trust, United Kingdom. The crucial computing support from all WLCG partners is acknowledged gratefully, in particular from CERN, the ATLAS Tier-1 facilities at TRIUMF (Canada), NDGF (Denmark, Norway, Sweden), CC-IN2P3 (France), KIT/GridKA (Germany), INFN-CNAF (Italy), NL-T1 (Netherlands), PIC (Spain), ASGC (Taiwan), RAL (UK), and BNL (USA), the Tier-2 facilities worldwide and large non-WLCG resource providers. Major contributors of computing resources are listed in Ref. [71].
[1] ATLAS Collaboration, Observation of a new particle in the search for the Standard Model Higgs boson with the ATLAS detector at the LHC, Phys. Lett. B 716, 1 (2012).

[2] CMS Collaboration, Observation of a new boson at a mass of $125 \mathrm{GeV}$ with the CMS experiment at the LHC, Phys. Lett. B 716, 30 (2012).
[3] M. J. Dugan, H. Georgi, and D. B. Kaplan, Anatomy of a composite Higgs model, Nucl. Phys. B254, 299 (1985).

[4] K. Agashe, R. Contino, and A. Pomarol, The minimal composite Higgs model, Nucl. Phys. B719, 165 (2005).

[5] M. Schmaltz and D. Tucker-Smith, Little Higgs theories, Annu. Rev. Nucl. Part. Sci. 55, 229 (2005). 
[6] K. Agashe et al., CERN LHC signals for warped electroweak neutral gauge bosons, Phys. Rev. D 76, 115015 (2007).

[7] K. Agashe, S. Gopalakrishna, T. Han, G.-Y. Huang, and A. Soni, LHC signals for warped electroweak charged gauge bosons, Phys. Rev. D 80, 075007 (2009).

[8] G. C. Branco et al., Theory and phenomenology of twoHiggs-doublet models, Phys. Rep. 516, 1 (2012).

[9] D. Pappadopulo, A. Thamm, R. Torre, and A. Wulzer, Heavy vector triplets: Bridging theory and data, J. High Energy Phys. 09 (2014) 060.

[10] ATLAS Collaboration, Combination of searches for heavy resonances decaying into bosonic and leptonic final states using $36 \mathrm{fb}^{-1}$ of proton-proton collision data at $\sqrt{\mathrm{s}}=$ $13 \mathrm{TeV}$ with the ATLAS detector, Phys. Rev. D 98, 052008 (2018).

[11] V. D. Barger, W.-Y. Keung, and E. Ma, Gauge model with light $W$ and $Z$ bosons, Phys. Rev. D 22, 727 (1980).

[12] ATLAS Collaboration, Search for heavy resonances decaying into a $W$ or $Z$ boson and a Higgs boson in final states with leptons and $b$-jets in $36 \mathrm{fb}^{-1}$ of $\sqrt{s}=13 \mathrm{TeV}$ $p p$ collisions with the ATLAS detector, J. High Energy Phys. 03 (2018) 174; Erratum, J. High Energy Phys. 11 (2018) 051.

[13] CMS Collaboration, Search for heavy resonances decaying into a vector boson and a Higgs boson in final states with charged leptons, neutrinos and $b$ quarks at $\sqrt{s}=13 \mathrm{TeV}, \mathrm{J}$. High Energy Phys. 11 (2018) 172.

[14] CMS Collaboration, Search for heavy resonances decaying into two Higgs bosons or into a Higgs boson and a $W$ or $Z$ boson in proton-proton collisions at $13 \mathrm{TeV}$, J. High Energy Phys. 01 (2019) 051.

[15] ATLAS Collaboration, Search for heavy resonances decaying to a $W$ or $Z$ boson and a Higgs boson in the $q \bar{q}^{(\prime)} b \bar{b}$ final state in $p p$ collisions at $\sqrt{s}=13 \mathrm{TeV}$ with the ATLAS detector, Phys. Lett. B 774, 494 (2017).

[16] CMS Collaboration, Search for heavy resonances that decay into a vector boson and a Higgs boson in hadronic final states at $\sqrt{s}=13 \mathrm{TeV}$, Eur. Phys. J. C 77, 636 (2017).

[17] ATLAS Collaboration, The ATLAS Experiment at the CERN large hadron collider, J. Instrum. 3, S08003 (2008).

[18] ATLAS Collaboration, ATLAS Insertable B-Layer Technical Design Report, Report No. ATLAS-TDR-19, 2010, https://cds.cern.ch/record/1291633.

[19] B. Abbott et al., Production and integration of the ATLAS insertable B-layer, J. Instrum. 13, T05008 (2018).

[20] ATLAS Collaboration, Performance of the ATLAS trigger system in 2015, Eur. Phys. J. C 77, 317 (2017).

[21] M. Cacciari, G. P. Salam, and G. Soyez, The anti- $k_{t}$ jet clustering algorithm, J. High Energy Phys. 04 (2008) 063.

[22] ATLAS Collaboration, Luminosity determination in $p p$ collisions at $\sqrt{s}=13 \mathrm{TeV}$ using the ATLAS detector at the LHC, Report No. ATLAS-CONF-2019-021, 2019, https://cds.cern.ch/record/2677054.

[23] J. Alwall, M. Herquet, F. Maltoni, O. Mattelaer, and T. Stelzer, MadGraph 5: Going beyond, J. High Energy Phys. 06 (2011) 128.

[24] R. D. Ball et al., Parton distributions with LHC data, Nucl. Phys. B867, 244 (2013).

[25] T. Sjöstrand, S. Mrenna, and P. Z. Skands, A brief introduction to PYTHIA 8.1, Comput. Phys. Commun. 178, 852 (2008).
[26] ATLAS Collaboration, ATLAS PYTHIA 8 tunes to $7 \mathrm{TeV}$ data, Report No. ATL-PHYS-PUB-2014-021, 2014, https:// cds.cern.ch/record/1966419.

[27] D. de Florian et al., Handbook of LHC Higgs cross sections: 4. Deciphering the nature of the Higgs sector, 2016, arXiv:1610.07922.

[28] S. Frixione, P. Nason, and G. Ridolfi, A positive-weight next-to-leading-order Monte Carlo for heavy flavour hadroproduction, J. High Energy Phys. 09 (2007) 126.

[29] ATLAS Collaboration, Comparison of Monte Carlo generator predictions for gap fraction and jet multiplicity observables in $t \bar{t}$ events, Report No. ATL-PHYS-PUB2014-005, 2014, https://cds.cern.ch/record/1703034.

[30] T. Gleisberg et al., Event generation with SHERPA 1.1, J. High Energy Phys. 02 (2009) 007.

[31] H.-L. Lai et al., New parton distributions for collider physics, Phys. Rev. D 82, 074024 (2010).

[32] M. Cacciari, M. Czakon, M. Mangano, A. Mitov, and P. Nason, Top-pair production at hadron colliders with next-tonext-to-leading logarithmic soft-gluon resummation, Phys. Lett. B 710, 612 (2012).

[33] M. Beneke, P. Falgari, S. Klein, and C. Schwinn, Hadronic top-quark pair production with NNLL threshold resummation, Nucl. Phys. B855, 695 (2012).

[34] P. Bärnreuther, M. Czakon, and A. Mitov, Percent-LevelPrecision Physics at the Tevatron: Next-to-Next-to-Leading Order QCD Corrections to $q \bar{q} \rightarrow t \bar{t}+X$, Phys. Rev. Lett. 109, 132001 (2012).

[35] M. Czakon and A. Mitov, NNLO corrections to top-pair production at hadron colliders: The all-fermionic scattering channels, J. High Energy Phys. 12 (2012) 054.

[36] M. Czakon and A. Mitov, NNLO corrections to top pair production at hadron colliders: The quark-gluon reaction, J. High Energy Phys. 01 (2013) 080.

[37] M. Czakon, P. Fiedler, and A. Mitov, Total Top-Quark PairProduction Cross Section at Hadron Colliders Through $O\left(\alpha_{S}^{4}\right)$, Phys. Rev. Lett. 110, 252004 (2013).

[38] M. Czakon and A. Mitov, Top++: A program for the calculation of the top-pair cross-section at hadron colliders, Comput. Phys. Commun. 185, 2930 (2014).

[39] D. J. Lange, The EvtGen particle decay simulation package, Nucl. Instrum. Methods Phys. Res., Sect. A 462, 152 (2001).

[40] ATLAS Collaboration, The PYTHIA 8 A3 tune description of ATLAS minimum bias and inelastic measurements incorporating the Donnachie-Landshoff diffractive model, Report No. ATL-PHYS-PUB-2016-017, 2016, https://cds.cern .ch/record/2206965.

[41] S. Agostinelli et al., GEANT4-A simulation toolkit, Nucl. Instrum. Methods Phys. Res., Sect. A 506, 250 (2003).

[42] ATLAS Collaboration, The ATLAS simulation infrastructure, Eur. Phys. J. C 70, 823 (2010).

[43] ATLAS Collaboration, Jet energy measurement with the ATLAS detector in proton-proton collisions at $\sqrt{s}=$ 7 TeV, Eur. Phys. J. C 73, 2304 (2013).

[44] M. Cacciari, G. P. Salam, and G. Soyez, FastJet user manual, Eur. Phys. J. C 72, 1896 (2012).

[45] ATLAS Collaboration, Boosted Higgs $(\rightarrow b \bar{b})$ boson identification with the ATLAS detector at $\sqrt{s}=13 \mathrm{TeV}$, Report No. ATLAS-CONF-2016-039, 2016, https://cds.cern.ch/ record/2206038. 
[46] ATLAS Collaboration, Improving jet substructure performance in ATLAS using track-caloclusters, Report No. ATLPHYS-PUB-2017-015, 2017, https://cds.cern.ch/record/ 2275636.

[47] ATLAS Collaboration, Search for diboson resonances in hadronic final states in $139 \mathrm{fb}^{-1}$ of $p p$ collisions at $\sqrt{s}=$ $13 \mathrm{TeV}$ with the ATLAS detector, J. High Energy Phys. 09 (2019) 091; Erratum, J. High Energy Phys. 06 (2020) 042.

[48] D. Krohn, J. Thaler, and L.-T. Wang, Jet trimming, J. High Energy Phys. 02 (2010) 084.

[49] S. D. Ellis and D. E. Soper, Successive combination jet algorithm for hadron collisions, Phys. Rev. D 48, 3160 (1993).

[50] ATLAS Collaboration, Identification of boosted, Hadronically-decaying $W$ and $Z$ bosons in $\sqrt{s}=13 \mathrm{TeV}$ Monte Carlo simulations for ATLAS, Report No. ATLPHYS-PUB-2015-033， 2015, https://cds.cern.ch/record/ 2041461.

[51] A. J. Larkoski, G. P. Salam, and J. Thaler, Energy correlation functions for jet substructure, J. High Energy Phys. 06 (2013) 108.

[52] A. J. Larkoski, I. Moult, and D. Neill, Power counting to better jet observables, J. High Energy Phys. 12 (2014) 009.

[53] M. Cacciari, G. P. Salam, and G. Soyez, The catchment area of jets, J. High Energy Phys. 04 (2008) 005.

[54] D. Krohn, J. Thaler, and L.-T. Wang, Jets with variable R, J. High Energy Phys. 06 (2009) 059.

[55] ATLAS Collaboration, Variable radius, exclusive- $k_{T}$, and center-of-mass subjet reconstruction for $\operatorname{Higgs}(\rightarrow b \bar{b})$ tagging in ATLAS, Report No. ATL-PHYS-PUB-2017-010, 2017, https://cds.cern.ch/record/2268678.

[56] ATLAS Collaboration, Performance of $b$-jet identification in the ATLAS experiment, J. Instrum. 11, P04008 (2016).

[57] ATLAS Collaboration, Optimisation of the ATLAS $b$-tagging performance for the 2016 LHC Run, Report No. ATL-PHYS-PUB-2016-012, 2016, https://cds.cern.ch/ record/2160731.

[58] ATLAS Collaboration, ATLAS b-jet identification performance and efficiency measurement with $t \bar{t}$ events in $\mathrm{pp}$ collisions at $\sqrt{s}=13 \mathrm{TeV}$, Eur. Phys. J. C 79, 970 (2019).
[59] ATLAS Collaboration, Electron and photon performance measurements with the ATLAS detector using the 20152017 LHC proton-proton collision data, J. Instrum. 14, P12006 (2019).

[60] ATLAS Collaboration, Muon reconstruction performance of the ATLAS detector in proton-proton collision data at $\sqrt{s}=13$ TeV, Eur. Phys. J. C 76, 292 (2016).

[61] ATLAS Collaboration, Selection of jets produced in $13 \mathrm{TeV}$ proton-proton collisions with the ATLAS detector, Report No. ATLAS-CONF-2015-029, 2015, https://cds.cern.ch/ record/2037702.

[62] A. Rogozhnikov, Reweighting with boosted decision trees, J. Phys. Conf. Ser. 762, 012036 (2016).

[63] ATLAS Collaboration, In situ calibration of large-radius jet energy and mass in $13 \mathrm{TeV}$ proton-proton collisions with the ATLAS detector, Eur. Phys. J. C 79, 135 (2019).

[64] ATLAS Collaboration, Performance of jet substructure techniques for large- $R$ jets in proton-proton collisions at $\sqrt{s}=7 \mathrm{TeV}$ using the ATLAS detector, J. High Energy Phys. 09 (2013) 076.

[65] ATLAS Collaboration, Jet mass reconstruction with the ATLAS detector in early Run 2 data, Report No. ATLASCONF-2016-035, 2016, https://cds.cern.ch/record/2200211.

[66] ATLAS Collaboration, Search for diboson resonances with boson-tagged jets in $p p$ collisions at $\sqrt{s}=13 \mathrm{TeV}$ with the ATLAS detector, Phys. Lett. B 777, 91 (2018).

[67] G. Avoni et al., The new LUCID-2 detector for luminosity measurement and monitoring in ATLAS, J. Instrum. 13, P07017 (2018).

[68] W. Verkerke and D. Kirkby, The RooFit toolkit for data modeling, 2003, arXiv:physics/0306116.

[69] G. Cowan, K. Cranmer, E. Gross, and O. Vitells, Asymptotic formulae for likelihood-based tests of new physics, Eur. Phys. J. C 71, 1554 (2011); Erratum, Eur. Phys. J. C 73, 2501 (2013).

[70] A. L. Read, Presentation of search results: The $C L_{S}$ technique, J. Phys. G 28, 2693 (2002).

[71] ATLAS Collaboration, ATLAS computing acknowledgements, Report No. ATL-SOFT-PUB-2020-001, https://cds .cern.ch/record/2717821.

G. Aad, ${ }^{102}$ B. Abbott, ${ }^{128}$ D. C. Abbott, ${ }^{103}$ A. Abed Abud, ${ }^{36}$ K. Abeling, ${ }^{53}$ D. K. Abhayasinghe, ${ }^{94}$ S. H. Abidi, ${ }^{166}$ O. S. AbouZeid, ${ }^{40}$ N. L. Abraham, ${ }^{155}$ H. Abramowicz, ${ }^{160}$ H. Abreu, ${ }^{159}$ Y. Abulaiti, ${ }^{6}$ B. S. Acharya, ${ }^{67 a, 67 b, b}$ B. Achkar, ${ }^{53}$ L. Adam, ${ }^{100}$ C. Adam Bourdarios, ${ }^{5}$ L. Adamczyk, ${ }^{84 a}$ L. Adamek, ${ }^{166}$ J. Adelman, ${ }^{121}$ M. Adersberger, ${ }^{114}$ A. Adiguzel, ${ }^{12 c}$ S. Adorni, ${ }^{54}$ T. Adye, ${ }^{143}$ A. A. Affolder, ${ }^{145}$ Y. Afik, ${ }^{159}$ C. Agapopoulou, ${ }^{65}$ M. N. Agaras, ${ }^{38}$ A. Aggarwal, ${ }^{119}$ C. Agheorghiesei, ${ }^{27 \mathrm{c}} \mathrm{J}$. A. Aguilar-Saavedra, ${ }^{139 \mathrm{f}, 139 \mathrm{a}, \mathrm{c}}$ A. Ahmad ${ }^{36}$ F. Ahmadov, ${ }^{80}$ W. S. Ahmed,${ }^{104}$ X. Ai,${ }^{18}$ G. Aielli, ${ }^{74 a, 74 b}$ S. Akatsuka, ${ }^{86}$ M. Akbiyik, ${ }^{100}$ T. P. A. Åkesson, ${ }^{97}$ E. Akilli, ${ }^{54}$ A. V. Akimov, ${ }^{111}$ K. Al Khoury, ${ }^{65}$ G. L. Alberghi, ${ }^{23 b, 23 a}$ J. Albert, ${ }^{175}$ M. J. Alconada Verzini, ${ }^{160}$ S. Alderweireldt, ${ }^{36}$ M. Aleksa ${ }^{36}$ I. N. Aleksandrov ${ }^{80}$ C. Alexa, ${ }^{27 b}$ T. Alexopoulos, ${ }^{10}$ A. Alfonsi, ${ }^{120}$ F. Alfonsi, ${ }^{23 b, 23 a}$ M. Alhroob, ${ }^{128}$ B. Ali,${ }^{141}$ S. Ali, ${ }^{157}$ M. Aliev, ${ }^{165}$ G. Alimonti, ${ }^{69 a}$ C. Allaire, ${ }^{36}$ B. M. M. Allbrooke, ${ }^{155}$ B. W. Allen, ${ }^{131}$ P. P. Allport, ${ }^{21}$ A. Aloisio, ${ }^{70 a, 70 b}$ F. Alonso, ${ }^{89}$ C. Alpigiani, ${ }^{147}$ E. Alunno Camelia, ${ }^{74 a, 74 b}$ M. Alvarez Estevez, ${ }^{99}$ M. G. Alviggi, ${ }^{70 a, 70 b}$ Y. Amaral Coutinho, ${ }^{81 b}$ A. Ambler, ${ }^{104}$ L. Ambroz, ${ }^{134}$ C. Amelung, ${ }^{26}$ D. Amidei, ${ }^{106}$ S. P. Amor Dos Santos, ${ }^{139 a}$ S. Amoroso, ${ }^{46}$ C. S. Amrouche, ${ }^{54}$ F. An, ${ }^{79}$ C. Anastopoulos, ${ }^{148}$ N. Andari, ${ }^{144}$ T. Andeen, ${ }^{11}$ J. K. Anders, ${ }^{20}$ S. Y. Andrean, ${ }^{45 a, 45 b}$ A. Andreazza, ${ }^{69 a, 69 b}$ V. Andrei, ${ }^{61 a}$ C. R. Anelli, ${ }^{175}$ S. Angelidakis, ${ }^{9}$ A. Angerami, ${ }^{39}$ A. V. Anisenkov, ${ }^{122 b, 122 a}$ A. Annovi, ${ }^{72 a}$ C. Antel, ${ }^{54}$ M. T. Anthony, ${ }^{148}$ E. Antipov, ${ }^{129}$ 
M. Antonelli, ${ }^{51}$ D. J. A. Antrim, ${ }^{170}$ F. Anulli, ${ }^{73 a}$ M. Aoki, ${ }^{82}$ J. A. Aparisi Pozo, ${ }^{173}$ M. A. Aparo, ${ }^{155}$ L. Aperio Bella, ${ }^{46}$ N. Aranzabal Barrio, ${ }^{36}$ V. Araujo Ferraz, ${ }^{81 a}$ R. Araujo Pereira, ${ }^{81 b}$ C. Arcangeletti, ${ }^{51}$ A. T. H. Arce, ${ }^{49}$ F. A. Arduh, ${ }^{89}$ J-F. Arguin, ${ }^{110}$ S. Argyropoulos, ${ }^{52}$ J.-H. Arling, ${ }^{46}$ A. J. Armbruster, ${ }^{36}$ A. Armstrong, ${ }^{170}$ O. Arnaez, ${ }^{166}$ H. Arnold, ${ }^{120}$ Z. P. Arrubarrena Tame, ${ }^{114}$ G. Artoni, ${ }^{134}$ H. Asada, ${ }^{117}$ K. Asai,${ }^{126}$ S. Asai, ${ }^{162}$ T. Asawatavonvanich, ${ }^{164}$ N. Asbah, ${ }^{59}$ E. M. Asimakopoulou, ${ }^{171}$ L. Asquith, ${ }^{155}$ J. Assahsah, ${ }^{35 \mathrm{~d}}$ K. Assamagan, ${ }^{29}$ R. Astalos ${ }^{28 \mathrm{a}}$ R. J. Atkin, ${ }^{33 \mathrm{a}}$ M. Atkinson, ${ }^{172}$ N. B. Atlay, ${ }^{19}$ H. Atmani, ${ }^{65}$ K. Augsten, ${ }^{141}$ V. A. Austrup,${ }^{181}$ G. Avolio, ${ }^{36}$ M. K. Ayoub, ${ }^{15 a}$ G. Azuelos, ${ }^{110, d}$ H. Bachacou, ${ }^{144}$

K. Bachas ${ }^{161}$ M. Backes, ${ }^{134}$ F. Backman, ${ }^{45 a, 45 b}$ P. Bagnaia,${ }^{73 a, 73 b}$ M. Bahmani,${ }^{85}$ H. Bahrasemani, ${ }^{151}$ A. J. Bailey ${ }^{173}$ V. R. Bailey, ${ }^{172}$ J. T. Baines, ${ }^{143}$ C. Bakalis, ${ }^{10}$ O. K. Baker, ${ }^{182}$ P. J. Bakker ${ }^{120}$ E. Bakos, ${ }^{16}$ D. Bakshi Gupta, ${ }^{8}$ S. Balaji, ${ }^{156}$ R. Balasubramanian, ${ }^{120}$ E. M. Baldin, ${ }^{122 b, 122 a}$ P. Balek, ${ }^{179}$ F. Balli, ${ }^{144}$ W. K. Balunas, ${ }^{134}$ J. Balz, ${ }^{100}$ E. Banas, ${ }^{85}$ M. Bandieramonte, ${ }^{138}$ A. Bandyopadhyay, ${ }^{24}$ Sw. Banerjee, ${ }^{180, e}$ L. Barak,${ }^{160}$ W. M. Barbe, ${ }^{38}$ E. L. Barberio, ${ }^{105}$ D. Barberis, ${ }^{55 b, 55 a}$ M. Barbero, ${ }^{102}$ G. Barbour, ${ }^{95}$ T. Barillari, ${ }^{115}$ M-S. Barisits, ${ }^{36}$ J. Barkeloo, ${ }^{131}$ T. Barklow, ${ }^{152}$ R. Barnea, ${ }^{159}$ B. M. Barnett, ${ }^{143}$ R. M. Barnett, ${ }^{18}$ Z. Barnovska-Blenessy, ${ }^{60 a}$ A. Baroncelli, ${ }^{60 a}$ G. Barone, ${ }^{29}$ A. J. Barr, ${ }^{134}$

L. Barranco Navarro, ${ }^{45 a, 45 b}$ F. Barreiro, ${ }^{99}$ J. Barreiro Guimarães da Costa,${ }^{15 a}$ U. Barron, ${ }^{160} \mathrm{~S}$. Barsov, ${ }^{137}$ F. Bartels,${ }^{61 a}$ R. Bartoldus, ${ }^{152}$ G. Bartolini, ${ }^{102}$ A. E. Barton, ${ }^{90}$ P. Bartos, ${ }^{28 a}$ A. Basalaev, ${ }^{46}$ A. Basan, ${ }^{100}$ A. Bassalat,${ }^{65, f}$ M. J. Basso, ${ }^{166}$ R. L. Bates,${ }^{57}$ S. Batlamous, ${ }^{35 e}$ J. R. Batley,${ }^{32}$ B. Batool, ${ }^{150}$ M. Battaglia, ${ }^{145}$ M. Bauce, ${ }^{73 a, 73 b}$ F. Bauer, ${ }^{144}$ P. Bauer, ${ }^{24}$ H. S. Bawa, ${ }^{31}$ A. Bayirli, ${ }^{12 c}$ J. B. Beacham, ${ }^{49}$ T. Beau, ${ }^{135}$ P. H. Beauchemin, ${ }^{169}$ F. Becherer, ${ }^{52}$ P. Bechtle,${ }^{24}$ H. C. Beck, ${ }^{53}$ H. P. Beck, ${ }^{20, g}$ K. Becker ${ }^{177}$ C. Becot, ${ }^{46}$ A. Beddall, ${ }^{12 d}$ A. J. Beddall, ${ }^{12 a}$ V. A. Bednyakov, ${ }^{80}$ M. Bedognetti, ${ }^{120}$ C. P. Bee, ${ }^{154}$ T. A. Beermann, ${ }^{181}$ M. Begalli, ${ }^{81 b}$ M. Begel, ${ }^{29}$ A. Behera, ${ }^{154}$ J. K. Behr, ${ }^{46}$ F. Beisiegel, ${ }^{24}$ M. Belfkir, ${ }^{5}$ A. S. Bell, ${ }^{95}$

G. Bella, ${ }^{160}$ L. Bellagamba, ${ }^{23 b}$ A. Bellerive, ${ }^{34}$ P. Bellos, ${ }^{9}$ K. Beloborodov, ${ }^{122 b, 122 a}$ K. Belotskiy, ${ }^{112}$ N. L. Belyaev, ${ }^{112}$ D. Benchekroun, ${ }^{35 a}$ N. Benekos, ${ }^{10}$ Y. Benhammou, ${ }^{160}$ D. P. Benjamin, ${ }^{6}$ M. Benoit, ${ }^{29}$ J. R. Bensinger, ${ }^{26}$ S. Bentvelsen, ${ }^{120}$ L. Beresford, ${ }^{134}$ M. Beretta, ${ }^{51}$ D. Berge,${ }^{19}$ E. Bergeaas Kuutmann, ${ }^{171}$ N. Berger, ${ }^{5}$ B. Bergmann, ${ }^{141}$ L. J. Bergsten, ${ }^{26}$ J. Beringer, ${ }^{18}$ S. Berlendis, ${ }^{7}$ G. Bernardi ${ }^{135}$ C. Bernius, ${ }^{152}$ F. U. Bernlochner, ${ }^{24}$ T. Berry, ${ }^{94}$ P. Berta, ${ }^{100}$ A. Berthold, ${ }^{48}$ I. A. Bertram, ${ }^{90}$ O. Bessidskaia Bylund, ${ }^{181}$ N. Besson, ${ }^{144}$ A. Bethani, ${ }^{101}$ S. Bethke, ${ }^{115}$ A. Betti, ${ }^{42}$ A. J. Bevan,${ }^{93}$ J. Beyer, ${ }^{115}$ D. S. Bhattacharya, ${ }^{176}$ P. Bhattarai, ${ }^{26}$ V. S. Bhopatkar, ${ }^{6}$ R. Bi, ${ }^{138}$ R. M. Bianchi, ${ }^{138}$ O. Biebel, ${ }^{114}$ D. Biedermann, ${ }^{19}$ R. Bielski, ${ }^{36}$ K. Bierwagen, ${ }^{100}$ N. V. Biesuz, ${ }^{72 a, 72 b}$ M. Biglietti, ${ }^{75 a}$ T. R. V. Billoud, ${ }^{141}$ M. Bindi, ${ }^{53}$ A. Bingul, ${ }^{12 d}$ C. Bini, ${ }^{73 a, 73 b}$ S. Biondi, ${ }^{23 b, 23 a}$ C. J. Birch-sykes, ${ }^{101}$ M. Birman, ${ }^{179}$ T. Bisanz,${ }^{53}$ J. P. Biswal, ${ }^{3}$ D. Biswas, ${ }^{180, e}$ A. Bitadze, ${ }^{101}$ C. Bittrich, ${ }^{48}$ K. Bjørke, ${ }^{133}$ T. Blazek, ${ }^{28 a}$ I. Bloch,${ }^{46}$ C. Blocker,${ }^{26}$ A. Blue,${ }^{57}$ U. Blumenschein, ${ }^{93}$ G. J. Bobbink, ${ }^{120}$

V. S. Bobrovnikov, ${ }^{122 b, 122 a}$ S. S. Bocchetta,${ }^{97}$ D. Boerner, ${ }^{46}$ D. Bogavac, ${ }^{14}$ A. G. Bogdanchikov, ${ }^{122 b, 122 a}$ C. Bohm, ${ }^{45 a}$ V. Boisvert, ${ }^{94}$ P. Bokan, ${ }^{171,171,53}$ T. Bold, ${ }^{84 a}$ A. E. Bolz ${ }^{61 b}$ M. Bomben, ${ }^{135}$ M. Bona,${ }^{93}$ J. S. Bonilla, ${ }^{131}$ M. Boonekamp, ${ }^{144}$ C. D. Booth,${ }^{94}$ A. G. Borbély, ${ }^{57}$ H. M. Borecka-Bielska, ${ }^{91}$ L. S. Borgna,${ }^{95}$ A. Borisov, ${ }^{123}$ G. Borissov, ${ }^{90}$ D. Bortoletto, ${ }^{134}$ D. Boscherini, ${ }^{23 b}$ M. Bosman, ${ }^{14}$ J. D. Bossio Sola, ${ }^{104}$ K. Bouaouda, ${ }^{35 a}$ J. Boudreau, ${ }^{138}$ E. V. Bouhova-Thacker, ${ }^{90}$

D. Boumediene, ${ }^{38}$ A. Boveia, ${ }^{127}$ J. Boyd ${ }^{36}$ D. Boye, ${ }^{33 \mathrm{c}}$ I. R. Boyko, ${ }^{80}$ A. J. Bozson, ${ }^{94}$ J. Bracinik, ${ }^{21}$ N. Brahimi, ${ }^{60 d}$ G. Brandt, ${ }^{181}$ O. Brandt, ${ }^{32}$ F. Braren, ${ }^{46}$ B. Brau, ${ }^{103}$ J. E. Brau, ${ }^{131}$ W. D. Breaden Madden, ${ }^{57}$ K. Brendlinger ${ }^{46}$ R. Brener, ${ }^{159}$ L. Brenner, ${ }^{36}$ R. Brenner, ${ }^{171}$ S. Bressler, ${ }^{179}$ B. Brickwedde, ${ }^{100}$ D. L. Briglin, ${ }^{21}$ D. Britton, ${ }^{57}$ D. Britzger, ${ }^{115}$ I. Brock, ${ }^{24}$ R. Brock, ${ }^{107}$ G. Brooijmans, ${ }^{39}$ W. K. Brooks, ${ }^{146 \mathrm{~d}}$ E. Brost,${ }^{29}$ P. A. Bruckman de Renstrom, ${ }^{85}$ B. Brüers,${ }^{46}$ D. Bruncko, ${ }^{28 b}$ A. Bruni, ${ }^{23 b}$ G. Bruni, ${ }^{23 b}$ M. Bruschi, ${ }^{23 b}$ N. Bruscino, ${ }^{73 a, 73 b}$ L. Bryngemark, ${ }^{152}$ T. Buanes, ${ }^{17}$ Q. Buat, ${ }^{154}$ P. Buchholz, ${ }^{150}$ A. G. Buckley, ${ }^{57}$ I. A. Budagov ${ }^{80}$ M. K. Bugge, ${ }^{133}$ F. Bührer, ${ }^{52}$ O. Bulekov, ${ }^{112}$ B. A. Bullard ${ }^{59}$ T. J. Burch, ${ }^{121}$ S. Burdin,,${ }^{91}$ C. D. Burgard ${ }^{120}$ A. M. Burger, ${ }^{129}$ B. Burghgrave,${ }^{8}$ J. T. P. Burr, ${ }^{46}$ C. D. Burton, ${ }^{11}$ J. C. Burzynski, ${ }^{103}$ V. Büscher, ${ }^{100}$ E. Buschmann, ${ }^{53}$ P. J. Bussey, ${ }^{57}$ J. M. Butler, ${ }^{25}$ C. M. Buttar, ${ }^{57}$ J. M. Butterworth, ${ }^{95}$ P. Butti, ${ }^{36}$ W. Buttinger, ${ }^{36}$ C. J. Buxo Vazquez, ${ }^{107}$ A. Buzatu, ${ }^{157}$ A. R. Buzykaev, ${ }^{122 b, 122 a}$ G. Cabras, ${ }^{23 b, 23 a}$ S. Cabrera Urbán, ${ }^{173}$ D. Caforio,${ }^{56}$ H. Cai, ${ }^{138}$ V. M. M. Cairo, ${ }^{152}$ O. Cakir, ${ }^{4 a}$ N. Calace,${ }^{36}$ P. Calafiura, ${ }^{18}$ G. Calderini, ${ }^{135}$ P. Calfayan, ${ }^{66}$ G. Callea, ${ }^{57}$ L. P. Caloba, ${ }^{81 b}$ A. Caltabiano, ${ }^{74 a, 74 b}$ S. Calvente Lopez, ${ }^{99}$ D. Calvet, ${ }^{38}$ S. Calvet, ${ }^{38}$ T. P. Calvet, ${ }^{102}$ M. Calvetti, ${ }^{72 a, 72 b}$ R. Camacho Toro, ${ }^{135}$ S. Camarda, ${ }^{36}$ D. Camarero Munoz, ${ }^{99}$ P. Camarri, ${ }^{74 a, 74 b}$ M. T. Camerlingo, ${ }^{75 a, 75 b}$ D. Cameron, ${ }^{133}$ C. Camincher, ${ }^{36}$ S. Campana, ${ }^{36}$ M. Campanelli, ${ }^{95}$ A. Camplani, ${ }^{40}$ V. Canale,${ }^{70 a, 70 b}$ A. Canesse, ${ }^{104}$ M. Cano Bret, ${ }^{78}$ J. Cantero, ${ }^{129}$ T. Cao, ${ }^{160}$ Y. Cao, ${ }^{172}$ M. D. M. Capeans Garrido, ${ }^{36}$ M. Capua, ${ }^{41 \mathrm{~b}, 41 \mathrm{a}}$ R. Cardarelli, ${ }^{7 \mathrm{a}}{ }^{\mathrm{F}}$. Cardillo, ${ }^{148}$ G. Carducci, ${ }^{41 b, 41 \mathrm{a}}$ I. Carli, ${ }^{142}$ T. Carli, ${ }^{36}$ G. Carlino, ${ }^{70 a}$ B. T. Carlson, ${ }^{138}$ E. M. Carlson, ${ }^{175,167 a}$ L. Carminati, ${ }^{69 a, 69 b}$ R. M. D. Carney, ${ }^{152}$ S. Caron, ${ }^{119}$ E. Carquin, ${ }^{146 \mathrm{~d}}$ S. Carrá, ${ }^{46}$ G. Carratta, ${ }^{23 b, 23 a}$ J. W. S. Carter,${ }^{166}$ T. M. Carter, ${ }^{50}$ M. P. Casado, ${ }^{14, \mathrm{~h}}$ A. F. Casha, ${ }^{166}$ E. G. Castiglia, ${ }^{182}$ F. L. Castillo, ${ }^{173}$ L. Castillo Garcia, ${ }^{14}$ V. Castillo Gimenez, ${ }^{173}$ N. F. Castro, ${ }^{139 a, 139 e}$ A. Catinaccio, ${ }^{36}$ J. R. Catmore, ${ }^{133}$ A. Cattai, ${ }^{36}$ V. Cavaliere, ${ }^{29}$ V. Cavasinni, ${ }^{72 a, 72 b}$ E. Celebi, ${ }^{12 b}$ F. Celli, ${ }^{134}$ K. Cerny, ${ }^{130}$ A. S. Cerqueira, ${ }^{81 a}$ 
A. Cerri, ${ }^{155}$ L. Cerrito, ${ }^{74 a, 74 b}$ F. Cerutti, ${ }^{18}$ A. Cervelli, ${ }^{23 b, 23 a}$ S. A. Cetin, ${ }^{12 b}$ Z. Chadi, ${ }^{35 a}$ D. Chakraborty, ${ }^{121}$ J. Chan, ${ }^{180}$ W. S. Chan, ${ }^{120}$ W. Y. Chan, ${ }^{91}$ J. D. Chapman, ${ }^{32}$ B. Chargeishvili, ${ }^{158 b}$ D. G. Charlton, ${ }^{21}$ T. P. Charman, ${ }^{93}$ M. Chatterjee, ${ }^{20}$ C. C. Chau, ${ }^{34}$ S. Che,${ }^{127}$ S. Chekanov, ${ }^{6}$ S. V. Chekulaev, ${ }^{167 a}$ G. A. Chelkov ${ }^{80, i}$ B. Chen,${ }^{79}$ C. Chen, ${ }^{60 a}$ C. H. Chen, ${ }^{79}$ H. Chen, ${ }^{15 \mathrm{c}}$ H. Chen, ${ }^{29}$ J. Chen, ${ }^{60 \mathrm{a}}$ J. Chen, ${ }^{39}$ J. Chen, ${ }^{26}$ S. Chen, ${ }^{136}$ S. J. Chen, ${ }^{15 \mathrm{c}}$ X. Chen, ${ }^{15 \mathrm{~b}}$ Y. Chen, ${ }^{60 \mathrm{a}}$ Y-H. Chen, ${ }^{46}$ H. C. Cheng, ${ }^{63 a}$ H. J. Cheng, ${ }^{15 a}$ A. Cheplakov, ${ }^{80}$ E. Cheremushkina, ${ }^{123}$ R. Cherkaoui El Moursli, ${ }^{35 e}$ E. Cheu, ${ }^{7}$ K. Cheung, ${ }^{64}$ T. J. A. Chevalérias, ${ }^{144}$ L. Chevalier, ${ }^{144}$ V. Chiarella, ${ }^{51}$ G. Chiarelli, ${ }^{72 \mathrm{a}}$ G. Chiodini, ${ }^{68 \mathrm{a}}$ A. S. Chisholm, ${ }^{21}$ A. Chitan, ${ }^{27 \mathrm{~b}}$ I. Chiu, ${ }^{162}$ Y. H. Chiu, ${ }^{175}$ M. V. Chizhov, ${ }^{80}$ K. Choi, ${ }^{11}$ A. R. Chomont, ${ }^{73 a, 73 b}$ Y. S. Chow, ${ }^{120}$ L. D. Christopher, ${ }^{33 e}$ M. C. Chu, ${ }^{63 \mathrm{a}}$ X. Chu, ${ }^{15 a, 15 \mathrm{~d}}$ J. Chudoba, ${ }^{140}$ J. J. Chwastowski, ${ }^{85}$ L. Chytka, ${ }^{130}$ D. Cieri, ${ }^{115}$ K. M. Ciesla, ${ }^{85}$ V. Cindro, ${ }^{92}$ I. A. Cioară ${ }^{27 b}$ A. Ciocio, ${ }^{18}$ F. Cirotto, ${ }^{70 a, 70 b}$ Z. H. Citron, ${ }^{179, j}$ M. Citterio, ${ }^{69 a}$ D. A. Ciubotaru, ${ }^{27 b}$ B. M. Ciungu, ${ }^{166}$ A. Clark, ${ }^{54}$ M. R. Clark, ${ }^{39}$ P. J. Clark, ${ }^{50}$ S. E. Clawson, ${ }^{101}$ C. Clement, ${ }^{45 a, 45 b}$ Y. Coadou, ${ }^{102}$ M. Cobal,,${ }^{67,67 \mathrm{c}}$ A. Coccaro, ${ }^{55 b}$ J. Cochran, ${ }^{79}$ R. Coelho Lopes De $S a,{ }^{103}$ H. Cohen, ${ }^{160}$ A. E. C. Coimbra ${ }^{36}$ B. Cole, ${ }^{39}$ A. P. Colijn, ${ }^{120}$ J. Collot, ${ }^{58}$ P. Conde Muiño, ${ }^{139 a, 139 \mathrm{~h}}$ S. H. Connell, ${ }^{33 \mathrm{c}}$ I. A. Connelly, ${ }^{57}$ S. Constantinescu, ${ }^{27 b}$ F. Conventi, ${ }^{70 a, k}$ A. M. Cooper-Sarkar, ${ }^{134}$ F. Cormier, ${ }^{174}$ K. J. R. Cormier, ${ }^{166}$ L. D. Corpe,${ }^{95}$ M. Corradi, ${ }^{73 a, 73 b}$ E. E. Corrigan, ${ }^{97}$ F. Corriveau, ${ }^{104,1}$ M. J. Costa, ${ }^{173}$ F. Costanza, ${ }^{5}$ D. Costanzo, ${ }^{148}$ G. Cowan, ${ }^{94}$ J. W. Cowley, ${ }^{32}$ J. Crane, ${ }^{101}$ K. Cranmer, ${ }^{125}$ R. A. Creager, ${ }^{136}$ S. Crépé-Renaudin, ${ }^{58}$ F. Crescioli, ${ }^{135}$ M. Cristinziani, ${ }^{24}$ V. Croft, ${ }^{169}$ G. Crosetti, ${ }^{41 b, 41 a}$ A. Cueto, ${ }^{5}$

T. Cuhadar Donszelmann, ${ }^{170}$ H. Cui, ${ }^{15 a, 15 d}$ A. R. Cukierman, ${ }^{152}$ W. R. Cunningham, ${ }^{57}$ S. Czekierda,${ }^{85}$ P. Czodrowski, ${ }^{36}$ M. M. Czurylo, ${ }^{61 b}$ M. J. Da Cunha Sargedas De Sousa, ${ }^{60 b}$ J. V. Da Fonseca Pinto, ${ }^{81 b}$ C. Da Via, ${ }^{101}$ W. Dabrowski, ${ }^{84 a}$ F. Dachs,${ }^{36}$ T. Dado,${ }^{47}$ S. Dahbi, ${ }^{33 e}$ T. Dai, ${ }^{106}$ C. Dallapiccola, ${ }^{103}$ M. Dam ${ }^{40}$ G. D'amen, ${ }^{29}$ V. D' Amico, ${ }^{75 a, 75 b}$ J. Damp, ${ }^{100}$ J. R. Dandoy, ${ }_{136}$ M. F. Daneri, ${ }^{30}$ M. Danninger, ${ }^{151}$ V. Dao,${ }^{36}$ G. Darbo, ${ }^{55 b}$ O. Dartsi, ${ }^{5}$ A. Dattagupta, ${ }^{131}$ T. Daubney, ${ }^{46}$ S. D’ Auria, ${ }^{69,69 b}$ C. David, ${ }^{167 b}$ T. Davidek, ${ }^{142}$ D. R. Davis, ${ }^{49}$ I. Dawson, ${ }^{148}$ K. De,${ }^{8}$ R. De Asmundis, ${ }^{70 a}$ M. De Beurs, ${ }^{120}$ S. De Castro, ${ }^{23 b, 23 a}$ N. De Groot,${ }^{119}$ P. de Jong, ${ }^{120}$ H. De la Torre, ${ }^{107}$ A. De Maria ${ }^{15 \mathrm{c}}$ D. De Pedis, ${ }^{73 a}$ A. De Salvo, ${ }^{73 a}$ U. De Sanctis, ${ }^{74 a, 74 b}$ M. De Santis, ${ }^{74 a}, 74 b$ A. De Santo, ${ }^{155}$ J. B. De Vivie De Regie, ${ }^{65}$ D. V. Dedovich, ${ }^{80}$ A. M. Deiana, ${ }^{42}$

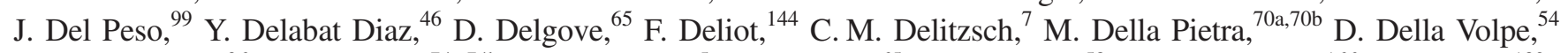
A. Dell'Acqua ${ }^{36}$ L. Dell'Asta, ${ }^{74 a, 74 b}$ M. Delmastro, ${ }^{5}$ C. Delporte, ${ }^{65}$ P. A. Delsart, ${ }^{58}$ D. A. DeMarco, ${ }^{166}$ S. Demers,${ }^{182}$ M. Demichev, ${ }^{80}$ G. Demontigny, ${ }^{110}$ S. P. Denisov, ${ }^{123}$ L. D'Eramo, ${ }^{121}$ D. Derendarz, ${ }^{85}$ J. E. Derkaoui, ${ }^{35 d}$ F. Derue, ${ }^{135}$

P. Dervan, ${ }^{91}$ K. Desch, ${ }^{24}$ K. Dette, ${ }^{166}$ C. Deutsch, ${ }^{24}$ M. R. Devesa, ${ }^{30}$ P. O. Deviveiros, ${ }^{36}$ F. A. Di Bello, ${ }^{73 a, 73 b}$

A. Di Ciaccio ${ }^{74 a, 74 b}$ L. Di Ciaccio, ${ }^{5}$ W. K. Di Clemente, ${ }^{136}$ C. Di Donato, ${ }^{70 a}, 70 b$ A. Di Girolamo, ${ }^{36}$ G. Di Gregorio, ${ }^{72 a, 72 b}$ B. Di Micco, ${ }^{75 a, 75 b}$ R. Di Nardo, ${ }^{75 a, 75 b}$ K. F. Di Petrillo, ${ }^{59}$ R. Di Sipio, ${ }^{166}$ C. Diaconu, ${ }^{102}$ F. A. Dias, ${ }^{120}$ T. Dias Do Vale, ${ }^{139 a}$ M. A. Diaz, ${ }^{146 a}$ F. G. Diaz Capriles ${ }^{24}$ J. Dickinson, ${ }^{18}$ M. Didenko, ${ }^{165}$ E. B. Diehl,${ }^{106}$ J. Dietrich, ${ }^{19}$ S. Díez Cornell, ${ }^{46}$ C. Diez Pardos, ${ }^{150}$ A. Dimitrievska, ${ }^{18}$ W. Ding, ${ }^{15 \mathrm{~b}}$ J. Dingfelder ${ }^{24}$ S. J. Dittmeier,${ }^{61 \mathrm{~b}}$ F. Dittus, ${ }^{36}$ F. Djama, ${ }^{102}$ T. Djobava, ${ }^{158 \mathrm{~b}}$ J. I. Djuvsland ${ }^{17}$ M. A. B. Do Vale, ${ }^{81 \mathrm{c}}$ M. Dobre, ${ }^{27 \mathrm{~b}}$ D. Dodsworth, ${ }^{26}$ C. Doglioni, ${ }^{97}$ J. Dolejsi, ${ }^{142}$ Z. Dolezal, ${ }^{142}$ M. Donadelli, ${ }^{81 \mathrm{~d}}$ B. Dong, ${ }^{60 \mathrm{c}}$ J. Donini, ${ }^{38}$ A. D’onofrio, ${ }^{15 \mathrm{c}}$ M. D’Onofrio,${ }^{91}$ J. Dopke, ${ }^{143}$ A. Doria, ${ }^{70 \mathrm{a}}$ M. T. Dova, ${ }^{89}$ A. T. Doyle ${ }^{57}$ E. Drechsler, ${ }^{151}$ E. Dreyer, ${ }^{151}$ T. Dreyer, ${ }^{53}$ A. S. Drobac, ${ }^{169}$ D. Du, ${ }^{60 b}$ T. A. du Pree, ${ }^{120}$ Y. Duan, ${ }^{60 d}$ F. Dubinin, ${ }^{111}$ M. Dubovsky, ${ }^{28 a}$ A. Dubreuil,${ }^{54}$ E. Duchovni, ${ }^{179}$ G. Duckeck,${ }^{114}$ O. A. Ducu, ${ }^{36}$ D. Duda,${ }^{115}$ A. Dudarev, ${ }^{36}$ A. C. Dudder, ${ }^{100}$ E. M. Duffield, ${ }^{18}$ M. D'uffizi, ${ }^{101}$ L. Duflot, ${ }^{65}$ M. Dührssen, ${ }^{36}$ C. Dülsen, ${ }^{181}$ M. Dumancic, ${ }^{179}$ A. E. Dumitriu, ${ }^{27 b}$ M. Dunford, ${ }^{61 a}$ A. Duperrin, ${ }^{102}$ H. Duran Yildiz, ${ }^{4 a}$ M. Düren ${ }^{56}$ A. Durglishvili, ${ }^{158 b}$ D. Duschinger, ${ }^{48}$ B. Dutta ${ }^{46}$ D. Duvnjak, ${ }^{1}$ G. I. Dyckes,${ }^{136}$ M. Dyndal, ${ }^{36}$ S. Dysch,${ }^{101}$ B. S. Dziedzic,${ }^{85}$ M. G. Eggleston, ${ }^{49}$ T. Eifert, ${ }^{8}$ G. Eigen, ${ }^{17}$ K. Einsweiler, ${ }^{18}$ T. Ekelof, ${ }^{171}$ H. El Jarrari, ${ }^{35 e}$ V. Ellajosyula, ${ }^{171}$ M. Ellert, ${ }^{171}$ F. Ellinghaus, ${ }^{181}$ A. A. Elliot,${ }^{93}$ N. Ellis, ${ }^{36}$ J. Elmsheuser, ${ }^{29}$ M. Elsing, ${ }^{36}$ D. Emeliyanov, ${ }^{143}$ A. Emerman, ${ }^{39}$ Y. Enari, ${ }^{162}$ M. B. Epland, ${ }^{49}$ J. Erdmann, ${ }^{47}$ A. Ereditato, ${ }^{20}$ P. A. Erland, ${ }^{85}$ M. Errenst, ${ }^{181}$ M. Escalier ${ }^{65}$ C. Escobar,${ }^{173}$ O. Estrada Pastor, ${ }^{173}$ E. Etzion, ${ }^{160}$ G. E. Evans, ${ }^{139 a, 139 b}$ H. Evans, ${ }^{66}$ M. O. Evans, ${ }^{155}$ A. Ezhilov, ${ }^{137}$ F. Fabbri, ${ }^{57}$ L. Fabbri, ${ }^{23 b, 23 a}$ V. Fabiani, ${ }^{119}$ G. Facini, ${ }^{177}$ R. M. Fakhrutdinov, ${ }^{123}$ S. Falciano, ${ }^{73 a}$ P. J. Falke, ${ }^{24}$ S. Falke, ${ }^{36}$ J. Faltova, ${ }^{142}$ Y. Fang, ${ }^{15 a}$ Y. Fang, ${ }^{15 a}$ G. Fanourakis, ${ }^{44}$ M. Fanti, ${ }^{69,69 b}$ M. Faraj, ${ }^{67,67 \mathrm{c}}$ A. Farbin, ${ }^{8}$ A. Farilla, ${ }^{75 a}$ E. M. Farina, ${ }^{71 a, 71 b}$ T. Farooque, ${ }^{107}$ S. M. Farrington, ${ }^{50}$ P. Farthouat, ${ }^{36}$ F. Fassi, ${ }^{35 e}$ P. Fassnacht, ${ }^{36}$ D. Fassouliotis, ${ }^{9}$ M. Faucci Giannelli, ${ }^{50}$ W. J. Fawcett, ${ }^{32}$ L. Fayard, ${ }^{65}$ O. L. Fedin, ${ }^{137, \mathrm{~m}}$ W. Fedorko, ${ }^{174}$ A. Fehr,${ }^{20}$ M. Feickert, ${ }^{172}$ L. Feligioni, ${ }^{102}$ A. Fell, ${ }^{148}$ C. Feng, ${ }^{60 b}$ M. Feng, ${ }^{49}$ M. J. Fenton, ${ }^{170}$ A. B. Fenyuk, ${ }^{123}$ S. W. Ferguson, ${ }^{43}$ J. Ferrando, ${ }^{46}$ A. Ferrante, ${ }^{172}$ A. Ferrari, ${ }^{171}$ P. Ferrari, ${ }^{120}$ R. Ferrari, ${ }^{71 a}$

D. E. Ferreira de Lima, ${ }^{61 \mathrm{~b}}$ A. Ferrer, ${ }^{173}$ D. Ferrere,${ }^{54}$ C. Ferretti, ${ }^{106}$ F. Fiedler, ${ }^{100}$ A. Filipčič,${ }^{92}$ F. Filthaut, ${ }^{119}$ K. D. Finelli, ${ }^{25}$ M. C. N. Fiolhais, ${ }^{139 a, 139 c, n}$ L. Fiorini, ${ }^{173}$ F. Fischer, ${ }^{114}$ J. Fischer, ${ }^{100}$ W. C. Fisher, ${ }^{107}$ T. Fitschen, ${ }^{21}$ I. Fleck, ${ }^{150}$ P. Fleischmann, ${ }^{106}$ T. Flick, ${ }^{181}$ B. M. Flierl, ${ }^{114}$ L. Flores, ${ }^{136}$ L. R. Flores Castillo, ${ }^{63 \mathrm{a}}$ F. M. Follega, ${ }^{76 a, 76 \mathrm{~b}}$ N. Fomin, ${ }^{17}$ 
J. H. Foo, ${ }^{166}$ G. T. Forcolin, ${ }^{76 a, 76 b}$ B. C. Forland, ${ }^{66}$ A. Formica, ${ }^{144}$ F. A. Förster, ${ }^{14}$ A. C. Forti, ${ }^{101}$ E. Fortin, ${ }^{102}$ M. G. Foti, ${ }^{134}$ D. Fournier, ${ }^{65}$ H. Fox,${ }^{90}$ P. Francavilla, ${ }^{72 a, 72 b}$ S. Francescato, ${ }^{73 a, 73 b}$ M. Franchini, ${ }^{23 b, 23 a}$ S. Franchino, ${ }^{61 a}$ D. Francis, ${ }^{36}$ L. Franco, ${ }^{5}$ L. Franconi, ${ }^{20}$ M. Franklin,${ }^{59}$ G. Frattari, ${ }^{73 a}, 73 \mathrm{~b}$ A. N. Fray, ${ }^{93}$ P. M. Freeman, ${ }^{21}$ B. Freund, ${ }^{110}$ W. S. Freund, ${ }^{81 b}$ E. M. Freundlich, ${ }^{47}$ D. C. Frizzell, ${ }^{128}$ D. Froidevaux,${ }^{36}$ J. A. Frost,${ }^{134}$ M. Fujimoto, ${ }^{126}$ C. Fukunaga, ${ }^{163}$

E. Fullana Torregrosa, ${ }^{173}$ T. Fusayasu, ${ }^{116}$ J. Fuster, ${ }^{173}$ A. Gabrielli, ${ }^{23 b, 23 a}$ A. Gabrielli, ${ }^{36}$ S. Gadatsch, ${ }^{54}$ P. Gadow, ${ }^{115}$ G. Gagliardi, ${ }^{55 b, 55 a}$ L. G. Gagnon, ${ }^{110}$ G. E. Gallardo, ${ }^{134}$ E. J. Gallas, ${ }^{134}$ B. J. Gallop, ${ }^{143}$ R. Gamboa Goni, ${ }^{93}$ K. K. Gan, ${ }^{127}$ S. Ganguly, ${ }^{179}$ J. Gao, ${ }^{60 a}$ Y. Gao, ${ }^{50}$ Y. S. Gao, ${ }^{31,0}$ F. M. Garay Walls, ${ }^{146 a}$ C. García, ${ }^{173}$ J. E. García Navarro, ${ }^{173}$ J. A. García Pascual, ${ }^{15 a}$ C. Garcia-Argos,${ }^{52}$ M. Garcia-Sciveres,${ }^{18}$ R. W. Gardner, ${ }^{37}$ N. Garelli, ${ }^{152}$ S. Gargiulo, ${ }^{52}$ C. A. Garner, ${ }^{166}$ V. Garonne, ${ }^{133}$ S. J. Gasiorowski, ${ }^{147}$ P. Gaspar, ${ }^{81 b}$ A. Gaudiello, ${ }^{55 b, 55 a}$ G. Gaudio, ${ }^{71 a}$ P. Gauzzi, ${ }^{73 a, 73 b}$ I. L. Gavrilenko, ${ }^{111}$ A. Gavrilyuk, ${ }^{124}$ C. Gay, ${ }^{174}$ G. Gaycken, ${ }^{46}$ E. N. Gazis, ${ }^{10}$ A. A. Geanta, ${ }^{27 b}$ C. M. Gee,${ }^{145}$ C. N. P. Gee, ${ }^{143}$ J. Geisen, ${ }^{97}$ M. Geisen, ${ }^{100}$ C. Gemme, ${ }^{55 b}$ M. H. Genest, ${ }^{58}$ C. Geng, ${ }^{106}$ S. Gentile, ${ }^{73 a, 73 b}$ S. George,${ }^{94}$ T. Geralis, ${ }^{44}$ L. O. Gerlach, ${ }^{53}$ P. Gessinger-Befurt, ${ }^{100}$ G. Gessner, ${ }^{47}$ S. Ghasemi, ${ }^{150}$ M. Ghasemi Bostanabad, ${ }^{175}$ M. Ghneimat, ${ }^{150}$ A. Ghosh, ${ }^{65}$ A. Ghosh, ${ }^{78}$ B. Giacobbe, ${ }^{23 b}$ S. Giagu, ${ }^{73 a, 73 b}$ N. Giangiacomi, ${ }^{23 b, 23 a}$ P. Giannetti, ${ }^{72 a}$ A. Giannini, ${ }^{70 a, 70 b}$ G. Giannini, ${ }^{14}$ S. M. Gibson, ${ }^{94}$ M. Gignac, ${ }^{145}$ D. T. Gil,${ }^{84 b}$ B. J. Gilbert, ${ }^{39}$ D. Gillberg, ${ }^{34}$ G. Gilles, ${ }^{181}$ N. E. K. Gillwald, ${ }^{46}$ D. M. Gingrich, ${ }^{3, d}$ M. P. Giordani, ${ }^{67,67 c}$ P. F. Giraud, ${ }^{144}$ G. Giugliarelli, ${ }^{67,67 c}$ D. Giugni, ${ }^{69 a}$ F. Giuli, ${ }^{74 a, 74 b}$ S. Gkaitatzis, ${ }^{161}$ I. Gkialas, ${ }^{9, p}$ E. L. Gkougkousis, ${ }^{14}$ P. Gkountoumis,${ }^{10}$ L. K. Gladilin, ${ }^{113}$ C. Glasman, ${ }^{99}$ J. Glatzer, ${ }^{14}$ P. C. F. Glaysher, ${ }^{46}$ A. Glazov, ${ }^{46}$ G. R. Gledhill, ${ }^{131}$ I. Gnesi, ${ }^{41 \mathrm{~b}, \mathrm{q}}$ M. Goblirsch-Kolb, ${ }^{26}$ D. Godin, ${ }^{10}$ S. Goldfarb, ${ }^{105}$ T. Golling, ${ }^{54}$ D. Golubkov, ${ }^{123}$ A. Gomes, ${ }^{139 a, 139 b}$ R. Goncalves Gama, ${ }^{53}$ R. Gonçalo, ${ }^{139 a, 139 c}$ G. Gonella, ${ }^{131}$ L. Gonella, ${ }^{21}$ A. Gongadze,${ }^{80}$ F. Gonnella, ${ }^{21}$ J. L. Gonski, ${ }^{39}$ S. González de la Hoz, ${ }^{173}$ S. Gonzalez Fernandez, ${ }^{14}$ R. Gonzalez Lopez,${ }^{91}$ C. Gonzalez Renteria, ${ }^{18}$ R. Gonzalez Suarez, ${ }^{171}$ S. Gonzalez-Sevilla, ${ }^{54}$ G. R. Gonzalvo Rodriguez, ${ }^{173}$ L. Goossens,${ }^{36}$ N. A. Gorasia, ${ }^{21}$ P. A. Gorbounov, ${ }^{124}$ H. A. Gordon, ${ }^{29}$ B. Gorini,${ }^{36}$ E. Gorini, ${ }^{68 a, 68 b}$ A. Gorišek, ${ }^{92}$ A. T. Goshaw, ${ }^{49}$ M. I. Gostkin, ${ }^{80}$ C. A. Gottardo, ${ }^{119}$ M. Gouighri, ${ }^{35 b}$ A. G. Goussiou, ${ }^{147}$ N. Govender, ${ }^{33 c}$ C. Goy, ${ }^{5}$ I. Grabowska-Bold,${ }^{84 a}$ E. C. Graham, ${ }^{91}$ J. Gramling, ${ }^{170}$ E. Gramstad, ${ }^{133}$ S. Grancagnolo, ${ }^{19}$ M. Grandi, ${ }^{155}$ V. Gratchev, ${ }^{137}$ P. M. Gravila, ${ }^{27 f}$ F. G. Gravili ${ }^{68 a, 68 b}$ C. Gray, ${ }^{57}$ H. M. Gray, ${ }^{18}$ C. Grefe,${ }^{24}$ K. Gregersen, ${ }^{97}$ I. M. Gregor, ${ }^{46}$ P. Grenier, ${ }^{152}$ K. Grevtsov, ${ }^{46}$ C. Grieco, ${ }^{14}$ N. A. Grieser, ${ }^{128}$ A. A. Grillo, ${ }^{145}$ K. Grimm, ${ }^{31, r}$ S. Grinstein, ${ }^{14, s}$ J.-F. Grivaz, ${ }^{65}$ S. Groh, ${ }^{100}$ E. Gross, ${ }^{179}$ J. Grosse-Knetter, ${ }^{53}$ Z. J. Grout, ${ }^{95}$ C. Grud, ${ }^{106}$ A. Grummer, ${ }^{118}$ J. C. Grundy, ${ }^{134}$ L. Guan, ${ }^{106}$ W. Guan, ${ }^{180}$ C. Gubbels, ${ }^{174}$ J. Guenther, ${ }^{36}$ A. Guerguichon, ${ }^{65}$ J. G. R. Guerrero Rojas, ${ }^{173}$ F. Guescini, ${ }^{115}$ D. Guest, ${ }^{170}$ R. Gugel, ${ }^{100}$ A. Guida, ${ }^{46}$ T. Guillemin, ${ }^{5}$ S. Guindon, ${ }^{36}$ J. Guo, ${ }^{60 \mathrm{c}}$ W. Guo, ${ }^{106}$ Y. Guo, ${ }^{60 \mathrm{a}}$ Z. Guo, ${ }^{102}$ R. Gupta, ${ }^{46}$ S. Gurbuz, ${ }^{12 \mathrm{c}}$ G. Gustavino, ${ }^{128}$ M. Guth, ${ }^{52}$ P. Gutierrez, ${ }^{128}$ C. Gutschow,${ }^{95}$ C. Guyot, ${ }^{144}$ C. Gwenlan, ${ }^{134}$ C. B. Gwilliam, ${ }^{91}$ E. S. Haaland, ${ }^{133}$ A. Haas, ${ }^{125}$ C. Haber, ${ }^{18}$ H. K. Hadavand, ${ }^{8}$ A. Hadef ${ }^{60 a}$ M. Haleem, ${ }^{176}$ J. Haley, ${ }^{129}$ J. J. Hall, ${ }^{148}$ G. Halladjian, ${ }^{107}$ G. D. Hallewell, ${ }^{102}$ K. Hamano, ${ }^{175}$ H. Hamdaoui, ${ }^{35 \mathrm{e}}$ M. Hamer, ${ }^{24}$ G. N. Hamity ${ }^{50}$ K. Han, ${ }^{60 a, t}$ L. Han, ${ }^{15 \mathrm{c}}$ L. Han, ${ }^{60 a}$ S. Han, ${ }^{18}$ Y. F. Han, ${ }^{166}$ K. Hanagaki, ${ }^{82, u}$ M. Hance, ${ }^{145}$ D. M. Handl, ${ }^{114}$ M. D. Hank,${ }^{37}$ R. Hankache, ${ }^{135}$ E. Hansen, ${ }^{97}$ J. B. Hansen, ${ }^{40}$ J. D. Hansen, ${ }^{40}$ M. C. Hansen, ${ }^{24}$ P. H. Hansen, ${ }^{40}$ E. C. Hanson, ${ }^{101}$ K. Hara, ${ }^{168}$ T. Harenberg, ${ }^{181}$ S. Harkusha, ${ }^{108}$ P. F. Harrison, ${ }^{177}$ N. M. Hartman, ${ }^{152}$ N. M. Hartmann, ${ }^{114}$ Y. Hasegawa, ${ }^{149}$ A. Hasib ${ }^{50}$ S. Hassani, ${ }^{144}$ S. Haug, ${ }^{20}$ R. Hauser, ${ }^{107}$ L. B. Havener, ${ }^{39}$ M. Havranek, ${ }^{141}$ C. M. Hawkes,${ }^{21}$ R. J. Hawkings, ${ }^{36}$ S. Hayashida ${ }^{117}$ D. Hayden, ${ }^{107}$ C. Hayes,${ }^{106}$ R. L. Hayes, ${ }^{174}$ C. P. Hays, ${ }^{134}$ J. M. Hays ${ }^{93}$ H. S. Hayward, ${ }^{91}$ S. J. Haywood, ${ }^{143}$ F. He, ${ }^{60 a}$ Y. He ${ }^{164}$ M. P. Heath ${ }^{50}$ V. Hedberg, ${ }^{97}$ S. Heer, ${ }^{24}$ A. L. Heggelund, ${ }^{133}$ C. Heidegger ${ }^{52}$ K. K. Heidegger, ${ }^{52}$ W. D. Heidorn, ${ }^{79}$ J. Heilman, ${ }^{34}$ S. Heim, ${ }^{46}$ T. Heim, ${ }^{18}$ B. Heinemann, ${ }^{46, v}$ J. J. Heinrich, ${ }^{131}$ L. Heinrich, ${ }^{36}$ J. Hejbal,${ }^{140}$ L. Helary, ${ }^{46}$ A. Held, ${ }^{125}$ S. Hellesund, ${ }^{133}$ C. M. Helling, ${ }^{145}$ S. Hellman, ${ }^{45 a, 45 b}$ C. Helsens, ${ }^{36}$ R. C. W. Henderson, ${ }^{90}$ Y. Heng, ${ }^{180}$ L. Henkelmann, ${ }^{32}$ A. M. Henriques Correia, ${ }^{36}$ H. Herde ${ }^{26}$ Y. Hernández Jiménez, ${ }^{33 e}$ H. Herr, ${ }^{100}$ M. G. Herrmann, ${ }^{114}$ T. Herrmann, ${ }^{48}$ G. Herten, ${ }^{52}$ R. Hertenberger ${ }^{114}$ L. Hervas, ${ }^{36}$ T. C. Herwig, ${ }^{136}$ G. G. Hesketh, ${ }^{95}$ N. P. Hessey, ${ }^{167 a}$ H. Hibi, ${ }^{83}$ S. Higashino, ${ }^{82}$ E. Higón-Rodriguez, ${ }^{173}$ K. Hildebrand ${ }^{37}$ J. C. Hill, ${ }^{32}$ K. K. Hill, ${ }^{29}$ K. H. Hiller ${ }^{46}$ S. J. Hillier ${ }^{21}$ M. Hils ${ }^{48}{ }^{\text {I. Hinchliffe }},{ }^{18}$ F. Hinterkeuser, ${ }^{24}$ M. Hirose, ${ }^{132}$ S. Hirose ${ }^{168}$ D. Hirschbuehl,${ }^{181}$ B. Hiti, ${ }^{92}$ O. Hladik, ${ }^{140}$ J. Hobbs, ${ }^{154}$ N. Hod, ${ }^{179}$ M. C. Hodgkinson, ${ }^{148}$ A. Hoecker, ${ }^{36}$ D. Hohn, ${ }^{52}$ D. Hohov ${ }^{65}$ T. Holm,${ }^{24}$ T. R. Holmes, ${ }^{37}$ M. Holzbock, ${ }^{115}$ L. B. A. H. Hommels,${ }^{32}$ T. M. Hong, ${ }^{138}$ J. C. Honig, ${ }^{52}$ A. Hönle, ${ }^{115}$ B. H. Hooberman, ${ }^{172}$ W. H. Hopkins, ${ }^{6}$ Y. Horii,${ }^{117}$ P. Horn, ${ }^{48}$ L. A. Horyn, ${ }^{37}$ S. Hou, ${ }^{157}$ A. Hoummada, ${ }^{35 a}$ J. Howarth,${ }^{57}$ J. Hoya,${ }^{89}$ M. Hrabovsky, ${ }^{130}$ J. Hrdinka, ${ }^{77}$ J. Hrivnac, ${ }^{65}$ A. Hrynevich, ${ }^{109}$ T. Hryn'ova, ${ }^{5}$ P. J. Hsu, ${ }^{64}$ S.-C. Hsu, ${ }^{147}$ Q. Hu ${ }^{29}$ S. Hu,${ }^{60 c}$ Y. F. Hu, ${ }^{15,15 \mathrm{~d}, \mathrm{w}}$ D. P. Huang, ${ }^{95}$ X. Huang, ${ }^{15 \mathrm{c}}$ Y. Huang, ${ }^{60 \mathrm{a}}$ Y. Huang, ${ }^{15 a}$ Z. Hubacek, ${ }^{141}$ F. Hubaut, ${ }^{102}$ M. Huebner, ${ }^{24}$ F. Huegging, ${ }^{24}$ T. B. Huffman, ${ }^{134}$ M. Huhtinen, ${ }^{36}$ R. Hulsken, ${ }^{58}$ R. F. H. Hunter, ${ }^{34}$ P. Huo, ${ }^{154}$ N. Huseynov, ${ }^{80, x}$ J. Huston, ${ }^{107}$ J. Huth, ${ }^{59}$ R. Hyneman, ${ }^{152}$ S. Hyrych, ${ }^{28 a}$ G. Iacobucci, ${ }^{54}$ 
G. Iakovidis, ${ }^{29}$ I. Ibragimov, ${ }^{150}$ L. Iconomidou-Fayard, ${ }^{65}$ P. Iengo,${ }^{36}$ R. Ignazzi, ${ }^{40}$ O. Igonkina, ${ }^{120, a, y}$ R. Iguchi, ${ }^{162}$ T. Iizawa, ${ }^{54}$ Y. Ikegami, ${ }^{82}$ M. Ikeno, ${ }^{82}$ N. Ilic,${ }^{119,166,1}$ F. Iltzsche, ${ }^{48}$ H. Imam, ${ }^{35 a}$ G. Introzzi, ${ }^{71 a, 71 b}$ M. Iodice, ${ }^{75 a}$

K. Iordanidou, ${ }^{167 a}$ V. Ippolito, ${ }^{73 a, 73 b}$ M. F. Isacson, ${ }^{171}$ M. Ishino, ${ }^{162}$ W. Islam, ${ }^{129}$ C. Issever, ${ }^{19,46} \mathrm{~S}$. Istin, ${ }^{159}$ J. M. Iturbe Ponce, ${ }^{63 a}$ R. Iuppa,${ }^{76 a, 76 b}$ A. Ivina, ${ }^{179}$ J. M. Izen, ${ }^{43}$ V. Izzo, ${ }^{70 a}$ P. Jacka, ${ }^{140}$ P. Jackson, ${ }^{1}$ R. M. Jacobs, ${ }^{46}$ B. P. Jaeger, ${ }^{151}$ V. Jain, ${ }^{2}$ G. Jäkel, ${ }^{181}$ K. B. Jakobi, ${ }^{100}$ K. Jakobs, ${ }^{52}$ T. Jakoubek, ${ }^{179}$ J. Jamieson, ${ }^{57}$ K. W. Janas, ${ }^{84 a}$ R. Jansky, ${ }^{54}$ M. Janus, ${ }^{53}$ P. A. Janus, ${ }^{84 a}$ G. Jarlskog, ${ }^{97}$ A. E. Jaspan, ${ }^{91}$ N. Javadov, ${ }^{80, x}$ T. Javůrek, ${ }^{36}$ M. Javurkova, ${ }^{103}$ F. Jeanneau, ${ }^{144}$ L. Jeanty, ${ }^{131}$ J. Jejelava, ${ }^{158 a}$ P. Jenni, ${ }^{52, z}$ N. Jeong, ${ }^{46}$ S. Jézéquel, ${ }^{5} \mathrm{H}$. Ji, ${ }^{180}$ J. Jia, ${ }^{154}$ Z. Jia, ${ }^{15 c} \mathrm{H}$. Jiang, ${ }^{79}$ Y. Jiang, ${ }^{60 a}$ Z. Jiang, ${ }^{152}$ S. Jiggins, ${ }^{52}$ F. A. Jimenez Morales, ${ }^{38}$ J. Jimenez Pena, ${ }^{115}$ S. Jin, ${ }^{15 c}$ A. Jinaru, ${ }^{27 b}$ O. Jinnouchi, ${ }^{164}$ H. Jivan, ${ }^{33 e}$ P. Johansson, ${ }^{148}$ K. A. Johns, ${ }^{7}$ C. A. Johnson, ${ }^{66}$ E. Jones, ${ }^{177}$ R. W. L. Jones, ${ }^{90}$ S. D. Jones, ${ }^{155}$ T. J. Jones, ${ }^{91}$ J. Jongmanns, ${ }^{61 a}$ J. Jovicevic, ${ }^{36}$ X. Ju, ${ }^{18}$ J. J. Junggeburth, ${ }^{115}$ A. Juste Rozas, ${ }^{14, s}$ A. Kaczmarska, ${ }^{85}$ M. Kado, ${ }^{73 a}, 73 b$ H. Kagan, ${ }^{127}$ M. Kagan, ${ }^{152}$ A. Kahn, ${ }^{39}$ C. Kahra, ${ }^{100}$ T. Kaji, ${ }^{178}$ E. Kajomovitz, ${ }^{159}$ C. W. Kalderon, ${ }^{29}$ A. Kaluza, ${ }^{100}$ A. Kamenshchikov, ${ }^{123}$ M. Kaneda, ${ }^{162}$

N. J. Kang, ${ }^{145}$ S. Kang, ${ }^{79}$ Y. Kano, ${ }^{117}$ J. Kanzaki, ${ }^{82}$ L. S. Kaplan, ${ }^{180}$ D. Kar, ${ }^{33 e}$ K. Karava, ${ }^{134}$ M. J. Kareem, ${ }^{167 b}$ I. Karkanias, ${ }^{161}$ S. N. Karpov, ${ }^{80}$ Z. M. Karpova,${ }^{80}$ V. Kartvelishvili, ${ }^{90}$ A. N. Karyukhin, ${ }^{123}$ E. Kasimi, ${ }^{161}$ A. Kastanas, ${ }^{45 a, 45 b}$ C. Kato, ${ }^{60 \mathrm{~d}}$ J. Katzy ${ }^{46}$ K. Kawade, ${ }^{149}$ K. Kawagoe, ${ }^{88}$ T. Kawaguchi, ${ }^{17}$ T. Kawamoto, ${ }^{144}$ G. Kawamura, ${ }^{53}$ E. F. Kay, ${ }^{175}$ S. Kazakos, ${ }^{14}$ V. F. Kazanin, ${ }^{122 b, 122 a}$ J. M. Keaveney, ${ }^{33 a}$ R. Keeler, ${ }^{175}$ J. S. Keller, ${ }^{34}$ E. Kellermann, ${ }^{97}$ D. Kelsey, ${ }^{155}$ J. J. Kempster, ${ }^{21}$ J. Kendrick, ${ }^{21}$ K. E. Kennedy, ${ }^{39}$ O. Kepka, ${ }^{140}$ S. Kersten, ${ }^{181}$ B. P. Kerševan, ${ }^{92}$ S. Ketabchi Haghighat, ${ }^{166}$ M. Khader, ${ }^{172}$ F. Khalil-Zada, ${ }^{13}$ M. Khandoga, ${ }^{144}$ A. Khanov, ${ }^{129}$ A. G. Kharlamov, ${ }^{122 b, 122 a}$ T. Kharlamova, ${ }^{122 b, 122 a}$ E. E. Khoda, ${ }^{174}$ A. Khodinov, ${ }^{165}$ T. J. Khoo, ${ }^{54}$ G. Khoriauli, ${ }^{176}$ E. Khramov,${ }^{80}$ J. Khubua, ${ }^{158 b}$ S. Kido, ${ }^{83}$ M. Kiehn, ${ }^{36}$ E. Kim, ${ }^{164}$ Y. K. Kim, ${ }^{37}$ N. Kimura, ${ }^{95}$ A. Kirchhoff, ${ }^{53}$ D. Kirchmeier, ${ }^{48}$ J. Kirk, ${ }^{143}$ A. E. Kiryunin, ${ }^{115}$ T. Kishimoto, ${ }^{162}$ D. P. Kisliuk, ${ }^{166}$ V. Kitali, ${ }^{46}$ C. Kitsaki, ${ }^{10}$ O. Kivernyk, ${ }^{24}$ T. Klapdor-Kleingrothaus, ${ }^{52}$ M. Klassen, ${ }^{61 a}$ C. Klein, ${ }^{34}$ M. H. Klein, ${ }^{106}$ M. Klein, ${ }^{91}$ U. Klein, ${ }^{91}$ K. Kleinknecht, ${ }^{100}$ P. Klimek, ${ }^{121}$ A. Klimentov, ${ }^{29}$ T. Klingl, ${ }^{24}$ T. Klioutchnikova, ${ }^{36}$ F. F. Klitzner, ${ }^{114}$ P. Kluit, ${ }^{120}$ S. Kluth, ${ }^{115}$ E. Kneringer, ${ }^{77}$ E. B. F. G. Knoops, ${ }^{102}$ A. Knue, ${ }^{52}$ D. Kobayashi, ${ }^{88}$ M. Kobel, ${ }^{48}$ M. Kocian, ${ }^{152}$ T. Kodama, ${ }^{162}$ P. Kodys, ${ }^{142}$ D. M. Koeck, ${ }^{155}$ P. T. Koenig, ${ }^{24}$ T. Koffas ${ }^{34}$ N. M. Köhler, ${ }^{36}$ M. Kolb, ${ }^{144}$ I. Koletsou, ${ }^{5}$ T. Komarek, ${ }^{130}$ T. Kondo, ${ }^{82}$ K. Köneke, ${ }^{52}$ A. X. Y. Kong, ${ }^{1}$ A. C. König, ${ }^{119}$ T. Kono, ${ }^{126}$ V. Konstantinides,${ }^{95}$ N. Konstantinidis, ${ }^{95}$ B. Konya, ${ }^{97}$ R. Kopeliansky, ${ }^{66}$ S. Koperny, ${ }^{84 a}$ K. Korcyl, ${ }^{85}$ K. Kordas, ${ }^{161}$ G. Koren, ${ }^{160}$ A. Korn, ${ }^{95}$ I. Korolkov, ${ }^{14}$ E. V. Korolkova, ${ }^{148}$ N. Korotkova, ${ }^{113}$ O. Kortner, ${ }^{115}$ S. Kortner, ${ }^{115}$ V. V. Kostyukhin, ${ }^{148,165}$

A. Kotsokechagia, ${ }^{65}$ A. Kotwal, ${ }^{49}$ A. Koulouris, ${ }^{10}$ A. Kourkoumeli-Charalampidi, ${ }^{71 a, 71 b}$ C. Kourkoumelis, ${ }^{9}$ E. Kourlitis, ${ }^{6}$ V. Kouskoura, ${ }^{29}$ R. Kowalewski, ${ }^{175}$ W. Kozanecki, ${ }^{101}$ A. S. Kozhin, ${ }^{123}$ V. A. Kramarenko, ${ }^{113}$ G. Kramberger, ${ }^{92}$ D. Krasnopevtsev ${ }^{60 a}$ M. W. Krasny, ${ }^{135}$ A. Krasznahorkay, ${ }^{36}$ D. Krauss, ${ }^{115}$ J. A. Kremer, ${ }^{100}$ J. Kretzschmar, ${ }^{91}$ P. Krieger, ${ }^{166}$ F. Krieter, ${ }^{14}$ A. Krishnan, ${ }^{61 \mathrm{~b}}$ M. Krivos, ${ }^{142}$ K. Krizka, ${ }^{18}$ K. Kroeninger, ${ }^{47}$ H. Kroha, ${ }^{15}$ J. Kroll, ${ }^{140}$ J. Kroll, ${ }^{136}$

K. S. Krowpman, ${ }^{107}$ U. Kruchonak, ${ }^{80}$ H. Krüger, ${ }^{24}$ N. Krumnack, ${ }^{79}$ M. C. Kruse, ${ }^{49}$ J. A. Krzysiak, ${ }^{85}$ A. Kubota, ${ }^{164}$ O. Kuchinskaia, ${ }^{165}$ S. Kuday, ${ }^{4 b}$ J. T. Kuechler, ${ }^{46}$ S. Kuehn, ${ }^{36}$ T. Kuhl, ${ }^{46}$ V. Kukhtin, ${ }^{80}$ Y. Kulchitsky, ${ }^{108, \text { aa }}$ S. Kuleshov, ${ }^{146 b}$ Y. P. Kulinich, ${ }^{172}$ M. Kuna, ${ }^{58}$ A. Kupco, ${ }^{140}$ T. Kupfer, ${ }^{47}$ O. Kuprash,${ }^{52}$ H. Kurashige, ${ }^{83}$ L. L. Kurchaninov, ${ }^{167 a}$ Y. A. Kurochkin, ${ }^{108}$ A. Kurova, ${ }^{112}$ M. G. Kurth, ${ }^{15 a, 15 d}$ E. S. Kuwertz, ${ }^{36}$ M. Kuze,${ }^{164}$ A. K. Kvam, ${ }^{147}$ J. Kvita, ${ }^{130}$ T. Kwan, ${ }^{104}$ F. La Ruffa ${ }^{41 b, 41 a}$ C. Lacasta, ${ }^{173}$ F. Lacava, ${ }^{73,73 b}$ D. P. J. Lack, ${ }^{101}$ H. Lacker, ${ }^{19}$ D. Lacour, ${ }^{135}$ E. Ladygin,${ }^{80}$ R. Lafaye, ${ }^{5}$ B. Laforge, ${ }^{135}$ T. Lagouri, ${ }^{146 c}$ S. Lai, ${ }^{53}$ I. K. Lakomiec, ${ }^{84 a}$ J. E. Lambert, ${ }^{128}$ S. Lammers, ${ }^{66}$ W. Lampl, ${ }^{7}$ C. Lampoudis, ${ }^{161}$ E. Lançon, ${ }^{29}$ U. Landgraf, ${ }^{52}$ M. P. J. Landon, ${ }^{93}$ M. C. Lanfermann, ${ }^{54}$ V. S. Lang, ${ }^{52}$ J. C. Lange, ${ }^{53}$ R. J. Langenberg, ${ }^{103}$

A. J. Lankford, ${ }^{170}$ F. Lanni, ${ }^{29}$ K. Lantzsch, ${ }^{24}$ A. Lanza, ${ }^{71 a}$ A. Lapertosa, ${ }^{55 b, 55 a}$ J. F. Laporte, ${ }^{144}$ T. Lari, ${ }^{69 a}$ F. Lasagni Manghi, ${ }^{23 b, 23 \mathrm{a}}$ M. Lassnig, ${ }^{36}$ V. Latonova, ${ }^{140}$ T. S. Lau, ${ }^{63 \mathrm{a}}$ A. Laudrain, ${ }^{100}$ A. Laurier, ${ }^{34}$ M. Lavorgna, ${ }^{70 a, 70 b}$ S. D. Lawlor, ${ }^{94}$ M. Lazzaroni, ${ }^{69 a, 69 b}$ B. Le, ${ }^{101}$ E. Le Guirriec, ${ }^{102}$ A. Lebedev,${ }^{79}$ M. LeBlanc, ${ }^{7}$ T. LeCompte, ${ }^{6}$

F. Ledroit-Guillon, ${ }^{58}$ A. C. A. Lee, ${ }^{95}$ C. A. Lee, ${ }^{29}$ G. R. Lee, ${ }^{17}$ L. Lee, ${ }^{59}$ S. C. Lee, ${ }^{157}$ S. Lee, ${ }^{79}$ B. Lefebvre, ${ }^{167 a}$ H. P. Lefebvre, ${ }^{94}$ M. Lefebvre, ${ }^{175}$ C. Leggett, ${ }^{18}$ K. Lehmann, ${ }^{151}$ N. Lehmann, ${ }^{20}$ G. Lehmann Miotto, ${ }^{36}$ W. A. Leight, ${ }^{46}$ A. Leisos, ${ }^{161, b b}$ M. A. L. Leite, ${ }^{81 d}$ C. E. Leitgeb,${ }^{114}$ R. Leitner,${ }^{142}$ D. Lellouch,,${ }^{179, a}$ K. J. C. Leney, ${ }^{42}$ T. Lenz,${ }^{24}$ S. Leone, ${ }^{72 a}$ C. Leonidopoulos, ${ }^{50}$ A. Leopold, ${ }^{135}$ C. Leroy, ${ }^{110}$ R. Les, ${ }^{107}$ C. G. Lester, ${ }^{32}$ M. Levchenko, ${ }^{137}$ J. Levêque, ${ }^{5}$ D. Levin, ${ }^{106}$ L. J. Levinson, ${ }^{179}$ D. J. Lewis, ${ }^{21}$ B. Li,${ }^{15 b}$ B. Li $,{ }^{106} \mathrm{C}-\mathrm{Q} . \mathrm{Li},{ }^{60 \mathrm{c}, 60 \mathrm{~d}} \mathrm{~F} . \mathrm{Li},{ }^{60 \mathrm{c}} \mathrm{H} . \mathrm{Li}{ }^{60 \mathrm{a}} \mathrm{H} . \mathrm{Li},{ }^{60 \mathrm{~b}} \mathrm{~J} . \mathrm{Li},{ }^{60 \mathrm{c}} \mathrm{K} . \mathrm{Li},{ }^{147} \mathrm{~L} . \mathrm{Li},{ }^{60 \mathrm{c}}$

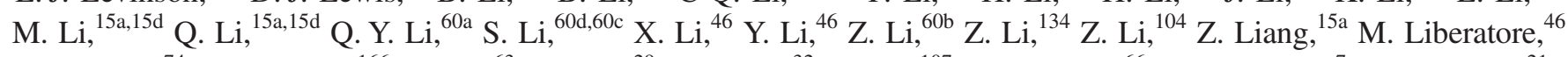
B. Liberti, ${ }^{74 a}$ A. Liblong, ${ }^{166}$ K. Lie, ${ }^{63 \mathrm{c}}$ S. Lim, ${ }^{29}$ C. Y. Lin, ${ }^{32}$ K. Lin, ${ }^{107}$ R. A. Linck, ${ }^{66}$ R. E. Lindley, ${ }^{7}$ J. H. Lindon, ${ }^{21}$ A. Linss ${ }^{46}$ A. L. Lionti, ${ }^{54}$ E. Lipeles,${ }^{136}$ A. Lipniacka, ${ }^{17}$ T. M. Liss, ${ }^{172, c c}$ A. Lister, ${ }^{174}$ J. D. Little, ${ }^{8}$ B. Liu ${ }^{79}$ B. L. Liu, ${ }^{151}$ H. B. Liu, ${ }^{29}$ J. B. Liu, ${ }^{60 \mathrm{a}}$ J. K. K. Liu, ${ }^{37}$ K. Liu, ${ }^{60 \mathrm{~d}}$ M. Liu, ${ }^{60 \mathrm{a}}$ M. Y. Liu, ${ }^{60 \mathrm{a}}$ P. Liu, ${ }^{15 \mathrm{a}}$ X. Liu, ${ }^{60 \mathrm{a}}$ Y. Liu, ${ }^{46}$ Y. Liu, ${ }^{15 a, 15 d}$ 
Y. L. Liu, ${ }^{106}$ Y. W. Liu, ${ }^{60 a}$ M. Livan, ${ }^{71 a, 71 b}$ A. Lleres, ${ }^{58}$ J. Llorente Merino, ${ }^{151}$ S. L. Lloyd, ${ }^{93}$ C. Y. Lo, ${ }^{63 b}$ E. M. Lobodzinska, ${ }^{46}$ P. Loch, ${ }^{7}$ S. Loffredo, ${ }^{74 a, 74 b}$ T. Lohse, ${ }^{19}$ K. Lohwasser, ${ }^{148}$ M. Lokajicek, ${ }^{140}$ J. D. Long, ${ }^{172}$ R. E. Long, ${ }^{90}$ I. Longarini, ${ }^{73 a, 73 b}$ L. Longo, ${ }^{36}$ K. A. Looper, ${ }^{127}$ I. Lopez Paz, ${ }^{101}$ A. Lopez Solis, ${ }^{148}$ J. Lorenz, ${ }^{114}$ N. Lorenzo Martinez, A. M. Lory, ${ }^{114}$ P. J. Lösel, ${ }^{114}$ A. Lösle, ${ }^{52}$ X. Lou, ${ }^{45 a, 45 b}$ X. Lou, ${ }^{15 a}$ A. Lounis, ${ }^{65}$ J. Love, ${ }^{6}$ P. A. Love, ${ }^{90}$ J. J. Lozano Bahilo, ${ }^{173}$ M. Lu, ${ }^{60 a}$ Y. J. Lu, ${ }^{64}$ H. J. Lubatti, ${ }^{147}$ C. Luci, ${ }^{73 a, 73 b}$ F. L. Lucio Alves, ${ }^{15 c}$ A. Lucotte ${ }^{58}$ F. Luehring, ${ }^{66}$ I. Luise, ${ }^{135}$ L. Luminari, ${ }^{73 a}$ B. Lund-Jensen, ${ }^{153}$ M. S. Lutz, ${ }^{160}$ D. Lynn, ${ }^{29}$ H. Lyons, ${ }^{91}$ R. Lysak, ${ }^{140}$ E. Lytken, ${ }^{97}$ F. Lyu, ${ }^{15 a}$ V. Lyubushkin, ${ }^{80}$ T. Lyubushkina, ${ }^{80}$ H. Ma, ${ }^{29}$ L. L. Ma ${ }^{60 b}$ Y. Ma,${ }^{95}$ D. M. Mac Donell, ${ }^{175}$ G. Maccarrone, ${ }^{51}$ A. Macchiolo, ${ }^{115}$ C. M. Macdonald, ${ }^{148}$ J. C. Macdonald, ${ }^{148}$ J. Machado Miguens, ${ }^{136}$ D. Madaffari, ${ }^{173}$ R. Madar, ${ }^{38}$ W. F. Mader, ${ }^{48}$ M. Madugoda Ralalage Don, ${ }^{129}$ N. Madysa, ${ }^{48}$ J. Maeda, ${ }^{83}$ T. Maeno, ${ }^{29}$ M. Maerker, ${ }^{48}$ V. Magerl, ${ }^{52}$ N. Magini, ${ }^{79}$ J. Magro, ${ }^{67 a, 67 c, d d}$ D. J. Mahon, ${ }^{39}$ C. Maidantchik, ${ }^{81 b}$ T. Maier, ${ }^{114}$ A. Maio, ${ }^{139 a, 139 b, 139 d}$ K. Maj, ${ }^{84 a}$ O. Majersky, ${ }^{28 a}$ S. Majewski, ${ }^{131}$ Y. Makida, ${ }^{82}$ N. Makovec, ${ }^{65}$ B. Malaescu, ${ }^{135}$ Pa. Malecki, ${ }^{85}$ V. P. Maleev, ${ }^{137}$ F. Malek, ${ }^{58}$ D. Malito, ${ }^{41 b, 41 a}$ U. Mallik, ${ }^{78}$ D. Malon, ${ }^{6}$ C. Malone, ${ }^{32}$ S. Maltezos, ${ }^{10}$ S. Malyukov, ${ }^{80}$ J. Mamuzic, ${ }^{173}$ G. Mancini, ${ }^{70 a, 70 b}$ I. Mandić, $^{92}$ L. Manhaes de Andrade Filho, ${ }^{81 a}$ I. M. Maniatis, ${ }^{161}$ J. Manjarres Ramos, ${ }^{48}$ K. H. Mankinen, ${ }^{97}$ A. Mann, ${ }^{114}$ A. Manousos, ${ }^{77}$ B. Mansoulie, ${ }^{144}$ I. Manthos, ${ }^{161}$ S. Manzoni, ${ }^{120}$ A. Marantis, ${ }^{161}$ G. Marceca ${ }^{30}$ L. Marchese, ${ }^{134}$ G. Marchiori, ${ }^{135}$ M. Marcisovsky, ${ }^{140}$ L. Marcoccia, ${ }^{74 a, 74 b}$ C. Marcon, ${ }^{97}$ M. Marjanovic, ${ }^{128}$ Z. Marshall, ${ }^{18}$ M. U. F. Martensson, ${ }^{171}$ S. Marti-Garcia, ${ }^{173}$ C. B. Martin, ${ }^{127}$ T. A. Martin, ${ }^{177}$ V. J. Martin, ${ }^{50}$ B. Martin dit Latour, ${ }^{17}$ L. Martinelli, ${ }^{75 a, 75 b}$ M. Martinez, ${ }^{14, s}$ P. Martinez Agullo, ${ }^{173}$ V. I. Martinez Outschoorn, ${ }^{103}$ S. Martin-Haugh, ${ }^{143}$ V. S. Martoiu, ${ }^{27 b}$ A. C. Martyniuk, ${ }^{95}$ A. Marzin, ${ }^{36}$ S. R. Maschek, ${ }^{115}$ L. Masetti, ${ }^{100}$ T. Mashimo, ${ }^{162}$ R. Mashinistov, ${ }^{111}$ J. Masik, ${ }^{101}$ A. L. Maslennikov, ${ }^{122 b, 122 a}$ L. Massa, ${ }^{23 b, 23 a}$ P. Massarotti, ${ }^{70 a, 70 b}$ P. Mastrandrea, ${ }^{72 a, 72 b}$ A. Mastroberardino, ${ }^{41 b, 41 a}$ T. Masubuchi, ${ }^{162}$ D. Matakias, ${ }^{29}$ A. Matic, ${ }^{114}$ N. Matsuzawa, ${ }^{162}$ P. Mättig, ${ }^{24}$ J. Maurer, ${ }^{27 b}$ B. Maček, ${ }^{92}$ D. A. Maximov, ${ }^{122 b, 122 a}$ R. Mazini, ${ }^{157}$ I. Maznas, ${ }^{161}$ S. M. Mazza, ${ }^{145}$ J. P. Mc Gowan, ${ }^{104}$ S. P. Mc Kee, ${ }^{106}$ T. G. McCarthy, ${ }^{115}$ W. P. McCormack, ${ }^{18}$ E. F. McDonald, ${ }^{105}$ A. E. Mcdougall, ${ }^{120}$ J. A. Mcfayden, ${ }^{18}$ G. Mchedlidze, ${ }^{158 b}$ M. A. McKay, ${ }^{42}$ K. D. McLean, ${ }^{175}$ S. J. McMahon, ${ }^{143}$ P. C. McNamara, ${ }^{105}$ C. J. McNicol, ${ }^{177}$ R. A. McPherson, ${ }^{175,1}$ J. E. Mdhluli, ${ }^{33 e}$ Z. A. Meadows, ${ }^{103}$ S. Meehan, ${ }^{36}$ T. Megy, ${ }^{38}$ S. Mehlhase, ${ }^{114}$ A. Mehta, ${ }^{91}$ B. Meirose, ${ }^{43}$ D. Melini, ${ }^{159}$

B. R. Mellado Garcia, ${ }^{33 e}$ J. D. Mellenthin, ${ }^{53}$ M. Melo, ${ }^{28 a}$ F. Meloni, ${ }^{46}$ A. Melzer, ${ }^{24}$ E. D. Mendes Gouveia, ${ }^{139 a, 139 e}$ A. M. Mendes Jacques Da Costa, ${ }^{21}$ L. Meng, ${ }^{36}$ X. T. Meng, ${ }^{106}$ S. Menke, ${ }^{115}$ E. Meoni, ${ }^{41 b, 41 a}$ S. Mergelmeyer, ${ }^{19}$ S. A. M. Merkt, ${ }^{138}$ C. Merlassino, ${ }^{134}$ P. Mermod, ${ }^{54}$ L. Merola, ${ }^{70 a, 70 b}$ C. Meroni, ${ }^{69 a}$ G. Merz, ${ }^{106}$ O. Meshkov, ${ }^{113,111}$ J. K. R. Meshreki, ${ }^{150}$ J. Metcalfe, ${ }^{6}$ A. S. Mete, ${ }^{6}$ C. Meyer, ${ }^{66}$ J-P. Meyer, ${ }^{144}$ M. Michetti, ${ }^{19}$ R. P. Middleton, ${ }^{143}$ L. Mijović, $^{50}$ G. Mikenberg, ${ }^{179}$ M. Mikestikova, ${ }^{140}$ M. Mikuž ${ }^{92}$ H. Mildner, ${ }^{148}$ A. Milic, ${ }^{166}$ C. D. Milke, ${ }^{42}$ D. W. Miller, ${ }^{37}$ A. Milov, ${ }^{179}$ D. A. Milstead, ${ }^{45 a, 45 b}$ R. A. Mina, ${ }^{152}$ A. A. Minaenko, ${ }^{123}$ I. A. Minashvili, ${ }^{158 b}$ A. I. Mincer, ${ }^{125}$ B. Mindur, ${ }^{84 a}$ M. Mineev, ${ }^{80}$ Y. Minegishi, ${ }^{162}$ Y. Mino, ${ }^{86}$ L. M. Mir, ${ }^{14}$ M. Mironova, ${ }^{134}$ K. P. Mistry, ${ }^{136}$ T. Mitani, ${ }^{178}$ J. Mitrevski, ${ }^{114}$ V. A. Mitsou, ${ }^{173}$ M. Mittal, ${ }^{60}$ O. Miu, ${ }^{166}$ A. Miucci, ${ }^{20}$ P. S. Miyagawa, ${ }^{93}$ A. Mizukami, ${ }^{82}$ J. U. Mjörnmark, ${ }^{97}$ T. Mkrtchyan, ${ }^{61 a}$ M. Mlynarikova, ${ }^{142}$ T. Moa, ${ }^{45 a, 45 b}$ S. Mobius, ${ }^{53}$ K. Mochizuki, ${ }^{110}$ P. Mogg, ${ }^{114}$ S. Mohapatra, ${ }^{39}$ R. Moles-Valls, ${ }^{24}$ K. Mönig, ${ }^{46}$ E. Monnier, ${ }^{102}$ A. Montalbano, ${ }^{151}$ J. Montejo Berlingen, ${ }^{36}$ M. Montella, ${ }^{95}$ F. Monticelli, ${ }^{89}$ S. Monzani, ${ }^{69 a}$ N. Morange, ${ }^{65}$ A. L. Moreira De Carvalho, ${ }^{139 a}$ D. Moreno, ${ }^{22 a}$ M. Moreno Llácer, ${ }^{173}$ C. Moreno Martinez, ${ }^{14}$ P. Morettini, ${ }^{55 b}$ M. Morgenstern, ${ }^{159}$ S. Morgenstern, ${ }^{48}$ D. Mori, ${ }^{151}$ M. Morii, ${ }^{59}$ M. Morinaga, ${ }^{178}$ V. Morisbak, ${ }^{133}$ A. K. Morley, ${ }^{36}$ G. Mornacchi, ${ }^{36}$ A. P. Morris, ${ }^{95}$ L. Morvaj, ${ }^{154}$ P. Moschovakos, ${ }^{36}$ B. Moser, ${ }^{120}$ M. Mosidze, ${ }^{158 b}$ T. Moskalets, ${ }^{144}$ P. Moskvitina, ${ }^{19}$ J. Moss, ${ }^{31, e e}$ E. J. W. Moyse, ${ }^{103}$ S. Muanza, ${ }^{102}$ J. Mueller, ${ }^{138}$ R. S. P. Mueller, ${ }^{114}$ D. Muenstermann, ${ }^{90}$ G. A. Mullier, ${ }^{97}$ D. P. Mungo, ${ }^{69 a, 69 b}$ J. L. Munoz Martinez, ${ }^{14}$ F. J. Munoz Sanchez, ${ }^{101}$ P. Murin, ${ }^{28 b}$ W. J. Murray, ${ }^{177,143}$

A. Murrone, ${ }^{69 a, 69 b}$ J. M. Muse, ${ }^{128}$ M. Muškinja, ${ }^{18}$ C. Mwewa, ${ }^{33 a}$ A. G. Myagkov, ${ }^{123, i}$ A. A. Myers, ${ }^{138}$ G. Myers, ${ }^{66}$ J. Myers, ${ }^{131}$ M. Myska, ${ }^{141}$ B. P. Nachman, ${ }^{18}$ O. Nackenhorst, ${ }^{47}$ A. Nag Nag, ${ }^{48}$ K. Nagai, ${ }^{134}$ K. Nagano, ${ }^{82}$ Y. Nagasaka, ${ }^{62}$ J. L. Nagle, ${ }^{29}$ E. Nagy, ${ }^{102}$ A. M. Nairz, ${ }^{36}$ Y. Nakahama, ${ }^{117}$ K. Nakamura, ${ }^{82}$ T. Nakamura, ${ }^{162}$ H. Nanjo, ${ }^{132}$ F. Napolitano, ${ }^{61 a}$ R. F. Naranjo Garcia, ${ }^{46}$ R. Narayan, ${ }^{42}$ I. Naryshkin, ${ }^{137}$ M. Naseri, ${ }^{34}$ T. Naumann, ${ }^{46}$ G. Navarro, ${ }^{22 a}$ P. Y. Nechaeva, ${ }^{111}$ F. Nechansky, ${ }^{46}$ T. J. Neep, ${ }^{21}$ A. Negri, ${ }^{71 a, 71 b}$ M. Negrini, ${ }^{23 b}$ C. Nellist, ${ }^{119}$ C. Nelson, ${ }^{104}$ M. E. Nelson, ${ }^{45 a, 45 b}$ S. Nemecek, ${ }^{140}$ M. Nessi, ${ }^{36, f f}$ M. S. Neubauer, ${ }^{172}$ F. Neuhaus, ${ }^{100}$ M. Neumann, ${ }^{181}$ R. Newhouse, ${ }^{174}$ P. R. Newman, ${ }^{21}$ C. W. Ng, ${ }^{138}$ Y. S. Ng, ${ }^{19}$ Y. W. Y. Ng, ${ }^{170}$ B. Ngair, ${ }^{35 e}$ H. D. N. Nguyen, ${ }^{102}$ T. Nguyen Manh, ${ }^{110}$ E. Nibigira, ${ }^{38}$ R. B. Nickerson, ${ }^{134}$ R. Nicolaidou, ${ }^{144}$ D. S. Nielsen, ${ }^{40}$ J. Nielsen, ${ }^{145}$ M. Niemeyer, ${ }^{53}$ N. Nikiforou, ${ }^{11}$ V. Nikolaenko, ${ }^{123, i}$ I. Nikolic-Audit, ${ }^{135}$ K. Nikolopoulos, ${ }^{21}$ P. Nilsson, ${ }^{29}$ H. R. Nindhito, ${ }^{54}$ A. Nisati, ${ }^{73 a}$ N. Nishu, ${ }^{60 c}$ R. Nisius, ${ }^{115}$ I. Nitsche, ${ }^{47}$ T. Nitta, ${ }^{178}$ T. Nobe, ${ }^{162}$ D. L. Noel, ${ }^{32}$ Y. Noguchi, ${ }^{86}$ I. Nomidis, ${ }^{135}$ M. A. Nomura, ${ }^{29}$ M. Nordberg, ${ }^{36}$ J. Novak, ${ }^{92}$ T. Novak, ${ }^{92}$ O. Novgorodova, ${ }^{48}$ R. Novotny, ${ }^{141}$ 
L. Nozka, ${ }^{130}$ K. Ntekas, ${ }^{170}$ E. Nurse, ${ }^{95}$ F. G. Oakham, ${ }^{34, \mathrm{~d}}$ H. Oberlack, ${ }^{115}$ J. Ocariz, ${ }^{135}$ A. Ochi, ${ }^{83}$ I. Ochoa, ${ }^{39}$ J. P. Ochoa-Ricoux, ${ }^{146 a}$ K. O'Connor, ${ }^{26}$ S. Oda, ${ }^{88}$ S. Odaka, ${ }^{82}$ S. Oerdek, ${ }^{53}$ A. Ogrodnik, ${ }^{84 a}$ A. Oh, ${ }^{101}$ C. C. Ohm, ${ }^{153}$ H. Oide, ${ }^{164}$ M. L. Ojeda, ${ }^{166}$ H. Okawa, ${ }^{168}$ Y. Okazaki, ${ }^{86}$ M. W. O'Keefe, ${ }^{91}$ Y. Okumura, ${ }^{162}$ A. Olariu, ${ }^{27 b}$

L. F. Oleiro Seabra, ${ }^{139 a}$ S. A. Olivares Pino, ${ }^{146 a}$ D. Oliveira Damazio, ${ }^{29}$ J. L. Oliver, ${ }^{1}$ M. J. R. Olsson, ${ }^{170}$ A. Olszewski, ${ }^{85}$ J. Olszowska, ${ }^{85}$ Ö. O. Öncel, ${ }^{24}$ D. C. O’Neil, ${ }^{151}$ A. P. O’neill,,${ }^{134}$ A. Onofre, ${ }^{139 a, 139 e}$ P. U. E. Onyisi, ${ }^{11}$ H. Oppen, ${ }^{133}$ R. G. Oreamuno Madriz, ${ }^{121}$ M. J. Oreglia, ${ }^{37}$ G. E. Orellana, ${ }^{89}$ D. Orestano, ${ }^{75 a, 75 b}$ N. Orlando, ${ }^{14}$ R. S. Orr, ${ }^{166}$ V. O'Shea ${ }^{57}$ R. Ospanov, ${ }^{60 a}$ G. Otero y Garzon, ${ }^{30}$ H. Otono, ${ }^{88}$ P. S. Ott, ${ }^{61 a}$ G. J. Ottino, ${ }^{18}$ M. Ouchrif, ${ }^{35 d}$ J. Ouellette, ${ }^{29}$ F. Ould-Saada, ${ }^{133}$ A. Ouraou, ${ }^{144}$ Q. Ouyang, ${ }^{15 a}$ M. Owen,${ }^{57}$ R. E. Owen, ${ }^{143}$ V. E. Ozcan, ${ }^{12 c}$ N. Ozturk, ${ }^{8}$ J. Pacalt,${ }^{130}$ H. A. Pacey, ${ }^{32}$ K. Pachal, ${ }^{49}$ A. Pacheco Pages, ${ }^{14}$ C. Padilla Aranda,${ }^{14}$ S. Pagan Griso, ${ }^{18}$ G. Palacino, ${ }^{66}$ S. Palazzo, ${ }^{50}$ S. Palestini, ${ }^{36}$ M. Palka, ${ }^{84 b}$ P. Palni,${ }^{84 a}$ C. E. Pandini, ${ }^{54}$ J. G. Panduro Vazquez, ${ }^{94}$ P. Pani, ${ }^{46}$ G. Panizzo, ${ }^{67 a, 67 c}$ L. Paolozzi,${ }^{54}$ C. Papadatos, ${ }^{110}$ K. Papageorgiou, ${ }^{9, p}$ S. Parajuli, ${ }^{42}$ A. Paramonov, ${ }^{6}$ C. Paraskevopoulos, ${ }^{10}$ D. Paredes Hernandez, ${ }^{63 b}$ S. R. Paredes Saenz, ${ }^{134}$ B. Parida, ${ }^{179}$ T. H. Park, ${ }^{166}$ A. J. Parker,${ }^{31}$ M. A. Parker, ${ }^{32}$ F. Parodi, ${ }^{55 b, 55 a}$ E. W. Parrish, ${ }^{121}$ J. A. Parsons, ${ }^{39}$ U. Parzefall, ${ }^{52}$ L. Pascual Dominguez, ${ }^{135}$ V. R. Pascuzzi, ${ }^{18}$ J. M. P. Pasner, ${ }^{145}$ F. Pasquali, ${ }^{120}$ E. Pasqualucci, ${ }^{73 a}$ S. Passaggio, ${ }^{55 b}$ F. Pastore,${ }^{94}$ P. Pasuwan, ${ }^{45 a, 45 b}$ S. Pataraia, ${ }^{100}$ J. R. Pater, ${ }^{101}$ A. Pathak, ${ }^{180, e}$ J. Patton, ${ }^{91}$ T. Pauly, ${ }^{36}$ J. Pearkes, ${ }^{152}$ B. Pearson, ${ }^{115}$ M. Pedersen, ${ }^{133}$ L. Pedraza Diaz, ${ }^{119}$ R. Pedro, ${ }^{139 a}$ T. Peiffer,${ }^{53}$ S. V. Peleganchuk, ${ }^{122 b, 122 a}$ O. Penc, ${ }^{140}$ H. Peng, ${ }^{60 a}$ B. S. Peralva, ${ }^{81 a}$ M. M. Perego, ${ }^{65}$ A. P. Pereira Peixoto, ${ }^{139 a}$ L. Pereira Sanchez, ${ }^{45 a, 45 b}$ D. V. Perepelitsa, ${ }^{29}$ E. Perez Codina, ${ }^{167 a}$ F. Peri, ${ }^{19}$ L. Perini, ${ }^{69 a, 69 b}$ H. Pernegger, ${ }^{36}$ S. Perrella, ${ }^{36}$ A. Perrevoort, ${ }^{120}$ K. Peters,${ }^{46}$ R. F. Y. Peters, ${ }^{101}$ B. A. Petersen, ${ }^{36}$ T. C. Petersen, ${ }^{40}$ E. Petit, ${ }^{102}$ V. Petousis, ${ }^{141}$ A. Petridis, ${ }^{1}$ C. Petridou, ${ }^{161}$ P. Petroff, ${ }^{65}$ F. Petrucci,${ }^{75 a, 75 b}$ M. Pettee,${ }^{182}$

N. E. Pettersson, ${ }^{103}$ K. Petukhova, ${ }^{142}$ A. Peyaud,${ }^{144}$ R. Pezoa, ${ }^{146 \mathrm{~d}}$ L. Pezzotti, ${ }^{71 \mathrm{a}, 7 \mathrm{~b}}$ T. Pham, ${ }^{105}$ P. W. Phillips, ${ }^{143}$ M. W. Phipps, ${ }^{172}$ G. Piacquadio, ${ }^{154}$ E. Pianori, ${ }^{18}$ A. Picazio, ${ }^{103}$ R. H. Pickles, ${ }^{101}$ R. Piegaia, ${ }^{30}$ D. Pietreanu, ${ }^{27 b}$ J. E. Pilcher, ${ }^{37}$ A. D. Pilkington, ${ }^{101}$ M. Pinamonti, ${ }^{67 a, 67 \mathrm{c}}$ J. L. Pinfold, ${ }^{3}$ C. Pitman Donaldson, ${ }^{95}$ M. Pitt, ${ }^{160}$ L. Pizzimento, ${ }^{74 a, 74 b}$ A. Pizzini, ${ }^{120}$ M.-A. Pleier, ${ }^{29}$ V. Plesanovs, ${ }^{52}$ V. Pleskot, ${ }^{142}$ E. Plotnikova, ${ }^{80}$ P. Podberezko, ${ }^{122 b, 122 a}$ R. Poettgen, ${ }^{97}$ R. Poggi,${ }^{54}$ L. Poggioli, ${ }^{135}$ I. Pogrebnyak, ${ }^{107}$ D. Pohl,${ }^{24}$ I. Pokharel,${ }^{53}$ G. Polesello, ${ }^{71 a}$ A. Poley, ${ }^{151,167 a}$ A. Policicchio, ${ }^{73 a, 73 b}$ R. Polifka, ${ }^{142}$ A. Polini, ${ }^{23 b}$ C. S. Pollard, ${ }^{46}$ V. Polychronakos, ${ }^{29}$ D. Ponomarenko, ${ }^{112}$ L. Pontecorvo,${ }^{36}$ S. Popa ${ }^{27 a}$ G. A. Popeneciu, ${ }^{27 d}$ L. Portales, ${ }^{5}$ D. M. Portillo Quintero, ${ }^{58}$ S. Pospisil, ${ }^{141}$ K. Potamianos, ${ }^{46}$ I. N. Potrap, ${ }^{80}$ C. J. Potter, ${ }^{32}$ H. Potti,${ }^{11}$ T. Poulsen, ${ }^{97}$ J. Poveda ${ }^{173}$ T. D. Powell, ${ }^{148}$ G. Pownall, ${ }^{46}$ M. E. Pozo Astigarraga,${ }^{36}$ A. Prades Ibanez, ${ }^{173}$ P. Pralavorio, ${ }^{102}$ M. M. Prapa ${ }^{44}$ S. Prell, ${ }^{79}$ D. Price, ${ }^{101}$ M. Primavera, ${ }^{68 a}$ M. L. Proffitt, ${ }^{147}$ N. Proklova, ${ }^{112}$ K. Prokofiev, ${ }^{63 c}$ F. Prokoshin, ${ }^{80}$ S. Protopopescu, ${ }^{29}$ J. Proudfoot, ${ }^{6}$ M. Przybycien, ${ }^{84 a}$ D. Pudzha, ${ }^{137}$ A. Puri, ${ }^{172}$ P. Puzo,${ }^{65}$ D. Pyatiizbyantseva, ${ }^{112}$ J. Qian, ${ }^{106}$ Y. Qin, ${ }^{101}$ A. Quadt, ${ }^{53}$ M. Queitsch-Maitland ${ }^{36}$ M. Racko, ${ }^{28 a}$ F. Ragusa, ${ }^{69 a, 69 b}$ G. Rahal,${ }^{98}$ J. A. Raine, ${ }^{54}$ S. Rajagopalan, ${ }^{29}$ A. Ramirez Morales, ${ }^{93}$ K. Ran, ${ }^{15 a}{ }^{15 d}$ D. M. Rauch, ${ }^{46}$ F. Rauscher, ${ }^{114}$ S. Rave, ${ }^{100}$ B. Ravina, ${ }^{57}$ I. Ravinovich, ${ }^{179}$ J. H. Rawling, ${ }^{101}$ M. Raymond, ${ }^{36}$ A. L. Read, ${ }^{133}$ N. P. Readioff, ${ }^{148}$ M. Reale, ${ }^{68 a, 68 b}$ D. M. Rebuzzi, ${ }^{71 a, 71 b}$ G. Redlinger, ${ }^{29}$ K. Reeves, ${ }^{43}$ D. Reikher, ${ }^{160}$ A. Reiss, ${ }^{100}$ A. Rej, ${ }^{150}$ C. Rembser, ${ }^{36}$ A. Renardi, ${ }^{46}$ M. Renda, ${ }^{27 b}$ M. B. Rendel, ${ }^{115}$ A. G. Rennie, ${ }^{57}$ S. Resconi, ${ }^{69 a}$ E. D. Resseguie, ${ }^{18}$ S. Rettie,${ }^{95}$ B. Reynolds, ${ }^{127}$ E. Reynolds, ${ }^{21}$ O. L. Rezanova, ${ }^{12 b, 122 a}$ P. Reznicek, ${ }^{142}$ E. Ricci, ${ }^{76 a, 76 \mathrm{~b}}$ R. Richter, ${ }^{115}$ S. Richter, ${ }^{46}$ E. Richter-Was,${ }^{84 \mathrm{~b}}$ M. Ridel, ${ }^{135}$ P. Rieck, ${ }^{115}$ O. Rifki, ${ }^{46}$ M. Rijssenbeek, ${ }^{154}$ A. Rimoldi, ${ }^{71 a, 71 b}$ M. Rimoldi, ${ }^{46}$ L. Rinaldi, ${ }^{23 b}$ T. T. Rinn, ${ }^{172}$ G. Ripellino, ${ }^{153}$ I. Riu, ${ }^{14}$ P. Rivadeneira ${ }^{46}$ J. C. Rivera Vergara, ${ }^{175}$ F. Rizatdinova, ${ }^{129}$ E. Rizvi, ${ }^{93}$ C. Rizzi, ${ }^{36}$ S. H. Robertson, ${ }^{104,1}$ M. Robin, ${ }^{46}$ D. Robinson, ${ }^{32}$ C. M. Robles Gajardo, ${ }^{146 \mathrm{~d}}$ M. Robles Manzano, ${ }^{100}$ A. Robson, ${ }^{57}$ A. Rocchi, ${ }^{74 a, 74 b}$ E. Rocco, ${ }^{100}$ C. Roda, ${ }^{72 a, 72 b}$ S. Rodriguez Bosca, ${ }^{173}$ A. Rodriguez Rodriguez, ${ }^{52}$ A. M. Rodríguez Vera, ${ }^{167 b}$ S. Roe, ${ }^{36}$ J. Roggel, ${ }^{181}$ O. Røhne, ${ }^{133}$ R. Röhrig, ${ }^{115}$ R. A. Rojas, ${ }^{146 \mathrm{~d}}$ B. Roland, ${ }^{52}$ C. P. A. Roland, ${ }^{66}$ J. Roloff, ${ }^{29}$ A. Romaniouk, ${ }^{112}$ M. Romano, ${ }^{23 b, 23 a}$ N. Rompotis, ${ }^{91}$ M. Ronzani, ${ }^{125}$ L. Roos, ${ }^{135}$ S. Rosati, ${ }^{73 a}$ G. Rosin, ${ }^{103}$ B. J. Rosser,${ }^{136}$ E. Rossi, ${ }^{46}$ E. Rossi, ${ }^{75 a}, 75 b$ E. Rossi ${ }^{70 a, 70 b}$ L. P. Rossi, ${ }^{55 b}$ L. Rossini,${ }^{46}$ R. Rosten, ${ }^{14}$ M. Rotaru, ${ }^{27 b}$ B. Rottler, ${ }^{52}$ D. Rousseau, ${ }^{65}$ G. Rovelli, ${ }^{71,71 b}$ A. Roy, ${ }^{11}$ D. Roy ${ }^{33 e}$ A. Rozanov, ${ }^{102}$ Y. Rozen, ${ }^{159}$ X. Ruan, ${ }^{33 e}$ T. A. Ruggeri, ${ }^{1}$ F. Rühr, ${ }^{52}$ A. Ruiz-Martinez, ${ }^{173}$ A. Rummler,${ }^{36}$ Z. Rurikova, ${ }^{52}$ N. A. Rusakovich, ${ }^{80}$ H. L. Russell, ${ }^{104}$ L. Rustige, ${ }^{38,47}$ J. P. Rutherfoord, ${ }^{7}$ E. M. Rüttinger, ${ }^{148}$ M. Rybar, ${ }^{39}$ G. Rybkin, ${ }^{65}$ E. B. Rye, ${ }^{133}$ A. Ryzhov, ${ }^{123}$ J. A. Sabater Iglesias, ${ }^{46}$ P. Sabatini, ${ }^{53}$ L. Sabetta, ${ }^{73 a, 73 b}$ S. Sacerdoti, ${ }^{65}$ H. F-W. Sadrozinski, ${ }^{145}$ R. Sadykov ${ }^{80}$ F. Safai Tehrani, ${ }^{73 a}$ B. Safarzadeh Samani, ${ }^{155}$ M. Safdari, ${ }^{152}$ P. Saha, ${ }^{121}$ S. Saha, ${ }^{104}$ M. Sahinsoy, ${ }_{115}$ A. Sahu ${ }_{181}$ M. Saimpert, ${ }^{36}$ M. Saito, ${ }^{162}$ T. Saito, ${ }^{162}$ H. Sakamoto, ${ }^{162}$ D. Salamani, ${ }^{54}$ G. Salamanna, ${ }^{75 a, 75 b}$ A. Salnikov, ${ }^{152}$ J. Salt, ${ }^{173}$ A. Salvador Salas, ${ }^{14}$ D. Salvatore, ${ }^{41 b, 41 a}$ F. Salvatore, ${ }^{155}$ A. Salvucci, ${ }^{63 a, 63 b, 63 c}$ A. Salzburger, ${ }^{36}$ J. Samarati, ${ }^{36}$ D. Sammel ${ }^{52}$ D. Sampsonidis ${ }^{161}$ D. Sampsonidou, ${ }^{161}$ J. Sánchez, ${ }^{173}$ A. Sanchez Pineda,${ }^{67,36,67 \mathrm{c}}$ H. Sandaker, ${ }^{133}$ C. O. Sander, ${ }^{46}$ I. G. Sanderswood, ${ }^{90}$ M. Sandhoff, ${ }^{181}$ C. Sandoval, ${ }^{22 b}$ D. P. C. Sankey, ${ }^{143}$ M. Sannino, ${ }^{55 b, 55 a}$ 
Y. Sano, ${ }^{117}$ A. Sansoni, ${ }^{51}$ C. Santoni, ${ }^{38}$ H. Santos, ${ }^{139 a, 139 b}$ S. N. Santpur, ${ }^{18}$ A. Santra, ${ }^{173}$ K. A. Saoucha, ${ }^{148}$ A. Sapronov, ${ }^{80}$ J. G. Saraiva, ${ }^{139 a, 139 d}$ O. Sasaki, ${ }^{82}$ K. Sato, ${ }^{168}$ F. Sauerburger, ${ }^{52}$ E. Sauvan, ${ }^{5}$ P. Savard, ${ }^{166, d}$ R. Sawada, ${ }^{162}$ C. Sawyer, ${ }^{143}$ L. Sawyer, ${ }^{96, g g}$ I. Sayago Galvan, ${ }^{173}$ C. Sbarra, ${ }^{23 b}$ A. Sbrizzi, ${ }^{67 a, 67 c}$ T. Scanlon, ${ }^{95}$ J. Schaarschmidt, ${ }^{147}$ P. Schacht, ${ }^{115}$ D. Schaefer, ${ }^{37}$ L. Schaefer, ${ }^{136}$ S. Schaepe, ${ }^{36}$ U. Schäfer, ${ }^{100}$ A. C. Schaffer, ${ }^{65}$ D. Schaile, ${ }^{114}$ R. D. Schamberger, ${ }^{154}$ E. Schanet, ${ }^{114}$ C. Scharf, ${ }^{19}$ N. Scharmberg, ${ }^{101}$ V. A. Schegelsky, ${ }^{137}$ D. Scheirich, ${ }^{142}$ F. Schenck, ${ }^{19}$ M. Schernau, ${ }^{170}$ C. Schiavi, ${ }^{55 b, 55 a}$ L. K. Schildgen, ${ }^{24}$ Z. M. Schillaci, ${ }^{26}$ E. J. Schioppa, ${ }^{68 a, 68 b}$ M. Schioppa, ${ }^{41 b, 41 a}$ K. E. Schleicher, ${ }^{52}$ S. Schlenker, ${ }^{36}$ K. R. Schmidt-Sommerfeld, ${ }^{115}$ K. Schmieden, ${ }^{36}$ C. Schmitt, ${ }^{100}$ S. Schmitt, ${ }^{46}$ L. Schoeffel, ${ }^{144}$ A. Schoening, ${ }^{61 b}$ P. G. Scholer, ${ }^{52}$ E. Schopf, ${ }^{134}$ M. Schott, ${ }^{100}$ J. F. P. Schouwenberg, ${ }^{119}$ J. Schovancova, ${ }^{36}$ S. Schramm, ${ }^{54}$ F. Schroeder, ${ }^{181}$ A. Schulte, ${ }^{100}$ H-C. Schultz-Coulon, ${ }^{61 \mathrm{a}}$ M. Schumacher, ${ }^{52}$ B. A. Schumm, ${ }^{145}$ Ph. Schune, ${ }^{144}$

A. Schwartzman, ${ }^{152}$ T. A. Schwarz, ${ }^{106}$ Ph. Schwemling, ${ }^{144}$ R. Schwienhorst, ${ }^{107}$ A. Sciandra, ${ }^{145}$ G. Sciolla, ${ }^{26}$ M. Scornajenghi, ${ }^{41 \mathrm{~b}, 41 \mathrm{a}}$ F. Scuri, ${ }^{72 \mathrm{a}}$ F. Scutti, ${ }^{105}$ L. M. Scyboz, ${ }^{115}$ C. D. Sebastiani, ${ }^{91}$ P. Seema, ${ }^{19}$ S. C. Seidel, ${ }^{118}$ A. Seiden, ${ }^{145}$ B. D. Seidlitz, ${ }^{29}$ T. Seiss, ${ }^{37}$ C. Seitz, ${ }^{46}$ J. M. Seixas, ${ }^{81 b}$ G. Sekhniaidze, ${ }^{70 a}$ S. J. Sekula, ${ }^{42}$ N. Semprini-Cesari, ${ }^{23 b, 23 a}$ S. Sen, ${ }^{49}$ C. Serfon, ${ }^{29}$ L. Serin, ${ }^{65}$ L. Serkin, ${ }^{67 a, 67 b}$ M. Sessa, ${ }^{60 a}$ H. Severini, ${ }^{128}$ S. Sevova, ${ }^{152}$ F. Sforza, ${ }^{55 b, 55 a}$ A. Sfyrla, ${ }^{54}$ E. Shabalina, ${ }^{53}$ J. D. Shahinian, ${ }^{145}$ N. W. Shaikh, ${ }^{45 a, 45 b}$ D. Shaked Renous, ${ }^{179}$ L. Y. Shan, ${ }^{15 a}$ M. Shapiro, ${ }^{18}$ A. Sharma, ${ }^{134}$ A. S. Sharma, ${ }^{1}$ P. B. Shatalov, ${ }^{124}$ K. Shaw, ${ }^{155}$ S. M. Shaw, ${ }^{101}$ M. Shehade, ${ }^{179}$ Y. Shen, ${ }^{128}$ A. D. Sherman, ${ }^{25}$ P. Sherwood, ${ }^{95}$ L. Shi, ${ }^{95}$ C. O. Shimmin, ${ }^{182}$ Y. Shimogama, ${ }^{178}$ M. Shimojima, ${ }^{116}$ J. D. Shinner, ${ }^{94}$ I. P. J. Shipsey, ${ }^{134}$ S. Shirabe, ${ }^{164}$ M. Shiyakova, ${ }^{80, \text { hh }}$ J. Shlomi, ${ }^{179}$ A. Shmeleva, ${ }^{11}$ M. J. Shochet, ${ }^{37}$ J. Shojaii, ${ }^{105}$ D. R. Shope, ${ }^{153}$ S. Shrestha, ${ }^{127}$ E. M. Shrif, ${ }^{33 e}$ M. J. Shroff, ${ }^{175}$ E. Shulga, ${ }^{179}$ P. Sicho, ${ }^{140}$ A. M. Sickles, ${ }^{172}$ E. Sideras Haddad, ${ }^{33 e}$ O. Sidiropoulou, ${ }^{36}$ A. Sidoti, ${ }^{23 b, 23 a}$ F. Siegert, ${ }^{48}$

Dj. Sijacki, ${ }^{16}$ M. Silva Jr., ${ }^{180}$ M. V. Silva Oliveira, ${ }^{36}$ S. B. Silverstein, ${ }^{45 a}$ S. Simion, ${ }^{65}$ R. Simoniello, ${ }^{100}$

C. J. Simpson-allsop, ${ }^{21}$ S. Simsek, ${ }^{12 b}$ P. Sinervo, ${ }^{166}$ V. Sinetckii, ${ }^{113}$ S. Singh, ${ }^{151}$ M. Sioli, ${ }^{23 b, 23 a}$ I. Siral, ${ }^{131}$ S. Yu. Sivoklokov, ${ }^{113}$ J. Sjölin, ${ }^{45 a, 45 b}$ A. Skaf, ${ }^{53}$ E. Skorda, ${ }^{97}$ P. Skubic, ${ }^{128}$ M. Slawinska, ${ }^{85}$ K. Sliwa, ${ }^{169}$ R. Slovak, ${ }^{142}$ V. Smakhtin, ${ }^{179}$ B. H. Smart, ${ }^{143}$ J. Smiesko, ${ }^{28 b}$ N. Smirnov, ${ }^{112}$ S. Yu. Smirnov, ${ }^{112}$ Y. Smirnov, ${ }^{112}$ L. N. Smirnova, ${ }^{113, i i}$ O. Smirnova, ${ }^{97}$ E. A. Smith, ${ }^{37}$ H. A. Smith, ${ }^{134}$ M. Smizanska, ${ }^{90}$ K. Smolek, ${ }^{141}$ A. Smykiewicz, ${ }^{85}$ A. A. Snesarev, ${ }^{111}$ H. L. Snoek, ${ }^{120}$ I. M. Snyder, ${ }^{131}$ S. Snyder, ${ }^{29}$ R. Sobie, ${ }^{175,1}$ A. Soffer, ${ }^{160}$ A. Søgaard, ${ }^{50}$ F. Sohns, ${ }^{53}$ C. A. Solans Sanchez, ${ }^{36}$ E. Yu. Soldatov, ${ }^{112}$ U. Soldevila, ${ }^{173}$ A. A. Solodkov, ${ }^{123}$ A. Soloshenko, ${ }^{80}$ O. V. Solovyanov, ${ }^{123}$ V. Solovyev, ${ }^{137}$ P. Sommer, ${ }^{148}$ H. Son, ${ }^{169}$ A. Sonay, ${ }^{14}$ W. Song, ${ }^{143}$ W. Y. Song, ${ }^{167 b}$ A. Sopczak, ${ }^{141}$ A. L. Sopio, ${ }^{95}$ F. Sopkova, ${ }^{28 b}$ S. Sottocornola, ${ }^{71 a, 71 b}$ R. Soualah, ${ }^{67 a, 67 c}$ A. M. Soukharev, ${ }^{122 b, 122 a}$ D. South, ${ }^{46}$ S. Spagnolo, ${ }^{68 a, 68 b}$ M. Spalla, ${ }^{115}$ M. Spangenberg, ${ }^{177}$ F. Spanò, ${ }^{94}$ D. Sperlich, ${ }^{52}$ T. M. Spieker, ${ }^{61 a}$ G. Spigo, ${ }^{36}$ M. Spina, ${ }^{155}$ D. P. Spiteri, ${ }^{57}$ M. Spousta, ${ }^{142}$ A. Stabile, ${ }^{69 a, 69 b}$ B. L. Stamas, ${ }^{121}$ R. Stamen, ${ }^{61 a}$ M. Stamenkovic, ${ }^{120}$ A. Stampekis, ${ }^{21}$ E. Stanecka, ${ }^{85}$ B. Stanislaus, ${ }^{134}$ M. M. Stanitzki, ${ }^{46}$ M. Stankaityte, ${ }^{134}$ B. Stapf, ${ }^{120}$ E. A. Starchenko, ${ }^{123}$ G. H. Stark, ${ }^{145}$ J. Stark, ${ }^{58}$ P. Staroba, ${ }^{140}$ P. Starovoitov, ${ }^{61 a}$ S. Stärz, ${ }^{104}$ R. Staszewski, ${ }^{85}$ G. Stavropoulos, ${ }^{44}$ M. Stegler, ${ }^{46}$ P. Steinberg, ${ }^{29}$ A. L. Steinhebel, ${ }^{131}$ B. Stelzer, ${ }^{151,167 a}$ H. J. Stelzer, ${ }^{138}$ O. Stelzer-Chilton, ${ }^{167 a}$ H. Stenzel, ${ }^{56}$ T. J. Stevenson, ${ }^{155}$ G. A. Stewart, ${ }^{36}$ M. C. Stockton, ${ }^{36}$ G. Stoicea, ${ }^{27 b}$ M. Stolarski, ${ }^{139 a}$ S. Stonjek, ${ }^{115}$ A. Straessner, ${ }^{48}$ J. Strandberg, ${ }^{153}$ S. Strandberg, ${ }^{45 a, 45 b}$ M. Strauss, ${ }^{128}$ T. Strebler, ${ }^{102}$ P. Strizenec, ${ }^{28 b}$ R. Ströhmer, ${ }^{176}$ D. M. Strom, ${ }^{131}$ R. Stroynowski, ${ }^{42}$ A. Strubig, ${ }^{50}$ S. A. Stucci, ${ }^{29}$ B. Stugu, ${ }^{17}$ J. Stupak, ${ }^{128}$ N. A. Styles, ${ }^{46}$ D. Su, ${ }^{152}$ W. Su, ${ }^{60 c, 147}$ X. Su, ${ }^{60 a}$ V. V. Sulin, ${ }^{111}$ M. J. Sullivan, ${ }^{91}$ D. M. S. Sultan, ${ }^{54}$ S. Sultansoy, ${ }^{4 c}$ T. Sumida, ${ }^{86}$ P. Sun, ${ }^{57}$ S. Sun, ${ }^{106}$ X. Sun, ${ }^{101}$ C. J. E. Suster, ${ }^{156}$ M. R. Sutton, ${ }^{155}$ S. Suzuki, ${ }^{82}$ M. Svatos, ${ }^{140}$ M. Swiatlowski, ${ }^{167 a}$ S. P. Swift, ${ }^{2}$ T. Swirski, ${ }^{176}$ A. Sydorenko, ${ }^{100}$ I. Sykora, ${ }^{28 a}$ M. Sykora, ${ }^{142}$ T. Sykora, ${ }^{142}$ D. Ta, ${ }^{100}$ K. Tackmann, ${ }^{46, j j}$ J. Taenzer, ${ }^{160}$ A. Taffard, ${ }^{170}$ R. Tafirout, ${ }^{167 a}$ E. Tagiev, ${ }^{123}$ R. Takashima, ${ }^{87}$ K. Takeda, ${ }^{83}$ T. Takeshita, ${ }^{149}$ E. P. Takeva, ${ }^{50}$ Y. Takubo, ${ }^{82}$ M. Talby, ${ }^{102}$ A. A. Talyshev, ${ }^{122 b, 122 \mathrm{a}}$ K. C. Tam, ${ }^{63 \mathrm{~b}}$ N. M. Tamir, ${ }^{160}$ J. Tanaka, ${ }^{162}$ R. Tanaka, ${ }^{65}$ S. Tapia Araya, ${ }^{172}$ S. Tapprogge, ${ }^{100}$ A. Tarek Abouelfadl Mohamed, ${ }^{107}$ S. Tarem, ${ }^{159}$ K. Tariq, ${ }^{60 b}$ G. Tarna, ${ }^{27 b, k k}$ G. F. Tartarelli, ${ }^{69}{ }^{\text {P. Tas, }}{ }^{142}$ M. Tasevsky, ${ }^{140}$ E. Tassi, ${ }^{41 \mathrm{~b}, 41 \mathrm{a}}$ A. Tavares Delgado, ${ }^{139 \mathrm{a}}$ Y. Tayalati, ${ }^{35 \mathrm{e}}$ A. J. Taylor, ${ }^{50}$ G. N. Taylor, ${ }^{105}$ W. Taylor, ${ }^{167 b}$ H. Teagle, ${ }^{91}$ A. S. Tee, ${ }^{90}$ R. Teixeira De Lima, ${ }^{152}$ P. Teixeira-Dias, ${ }^{94}$ H. Ten Kate, ${ }^{36}$ J. J. Teoh, ${ }^{120}$ K. Terashi, ${ }^{162}$ J. Terron, ${ }^{99}$ S. Terzo, ${ }^{14}$ M. Testa, ${ }^{51}$ R. J. Teuscher, ${ }^{166,1}$ S. J. Thais, ${ }^{182}$ N. Themistokleous, ${ }^{50}$ T. Theveneaux-Pelzer, ${ }^{46}$ F. Thiele, ${ }^{40}$ D. W. Thomas, ${ }^{94}$

J. O. Thomas, ${ }^{42}$ J. P. Thomas, ${ }^{21}$ E. A. Thompson, ${ }^{46}$ P. D. Thompson, ${ }^{21}$ E. Thomson, ${ }^{136}$ E. J. Thorpe, ${ }^{93}$ R. E. Ticse Torres, ${ }^{53}$

V. O. Tikhomirov, ${ }^{111,11}$ Yu. A. Tikhonov, ${ }^{122 b, 122 \mathrm{a}}$ S. Timoshenko, ${ }^{112}$ P. Tipton, ${ }^{182}$ S. Tisserant, ${ }^{102} \mathrm{~K}$. Todome, ${ }^{23 \mathrm{~b}, 23 \mathrm{a}}$ S. Todorova-Nova, ${ }^{142}$ S. Todt, ${ }^{48}$ J. Tojo, ${ }^{88}$ S. Tokár, ${ }^{28 a}$ K. Tokushuku, ${ }^{82}$ E. Tolley, ${ }^{127}$ R. Tombs, ${ }^{32}$ K. G. Tomiwa, ${ }^{33 e}$ M. Tomoto,${ }^{82,117}$ L. Tompkins, ${ }^{152}$ P. Tornambe, ${ }^{103}$ E. Torrence, ${ }^{131}$ H. Torres, ${ }^{48}$ E. Torró Pastor, ${ }^{173}$ M. Toscani, ${ }^{30}$ C. Tosciri, ${ }^{134}$ J. Toth, ${ }^{102, \mathrm{~mm}}$ D. R. Tovey, ${ }^{148}$ A. Traeet, ${ }^{17}$ C. J. Treado, ${ }^{125}$ T. Trefzger, ${ }^{176}$ F. Tresoldi, ${ }^{155}$ A. Tricoli, ${ }^{29}$ I. M. Trigger, ${ }^{167 a}$ S. Trincaz-Duvoid, ${ }^{135}$ D. A. Trischuk, ${ }^{174}$ W. Trischuk, ${ }^{166}$ B. Trocmé, ${ }^{58}$ A. Trofymov, ${ }^{65}$ C. Troncon, ${ }^{69}$ F. Trovato, ${ }^{155}$ 
L. Truong, ${ }^{33 \mathrm{c}}$ M. Trzebinski, ${ }^{85}$ A. Trzupek ${ }^{85}$ F. Tsai, ${ }^{46}$ J. C-L. Tseng, ${ }^{134}$ P. V. Tsiareshka, ${ }^{108, a a}$ A. Tsirigotis, ${ }^{161, b b}$ V. Tsiskaridze, ${ }^{154}$ E. G. Tskhadadze, ${ }^{158 a}$ M. Tsopoulou, ${ }^{161}$ I. I. Tsukerman, ${ }^{124}$ V. Tsulaia, ${ }^{18}$ S. Tsuno, ${ }^{82}$ D. Tsybychev, ${ }^{154}$ Y. Tu, ${ }^{63 \mathrm{~b}}$ A. Tudorache, ${ }^{27 \mathrm{~b}}$ V. Tudorache, ${ }^{27 \mathrm{~b}}$ T. T. Tulbure, ${ }^{27 \mathrm{a}}$ A. N. Tuna, ${ }^{59}$ S. Turchikhin, ${ }^{80}$ D. Turgeman, ${ }^{179}$ I. Turk Cakir, ${ }^{4, n n}$ R. J. Turner, ${ }^{21}$ R. Turra, ${ }^{69 a}$ P. M. Tuts, ${ }^{39}$ S. Tzamarias, ${ }^{161}$ E. Tzovara, ${ }^{100}$ K. Uchida, ${ }^{162}$ F. Ukegawa, ${ }^{168}$ G. Unal, ${ }^{36}$ M. Unal, ${ }^{11}$ A. Undrus, ${ }^{29}$ G. Unel, ${ }^{170}$ F. C. Ungaro, ${ }^{105}$ Y. Unno, ${ }^{82}$ K. Uno, ${ }^{162}$ J. Urban, ${ }^{28 b}$ P. Urquijo, ${ }^{105}$ G. Usai, ${ }^{8}$ Z. Uysal, ${ }^{12 \mathrm{~d}}$ V. Vacek, ${ }^{141}$ B. Vachon, ${ }^{104}$ K. O. H. Vadla, ${ }^{133}$ T. Vafeiadis, ${ }^{36}$ A. Vaidya ${ }^{95}$ C. Valderanis, ${ }^{114}$

E. Valdes Santurio, ${ }^{45 a, 45 b}$ M. Valente, ${ }^{54}$ S. Valentinetti, ${ }^{23 b, 23 a}$ A. Valero, ${ }^{173}$ L. Valéry, ${ }^{46}$ R. A. Vallance, ${ }^{21}$ A. Vallier, ${ }^{36}$ J. A. Valls Ferrer, ${ }^{173}$ T. R. Van Daalen, ${ }^{14}$ P. Van Gemmeren, ${ }^{6}$ S. Van Stroud, ${ }^{95}$ I. Van Vulpen, ${ }^{120}$ M. Vanadia, ${ }^{74 a, 74 b}$ W. Vandelli, ${ }^{36}$ M. Vandenbroucke, ${ }^{144}$ E. R. Vandewall, ${ }^{129}$ A. Vaniachine, ${ }^{165}$ D. Vannicola, ${ }^{73 a, 73 b}$ R. Vari, ${ }^{73 a}$ E. W. Varnes, ${ }^{7}$

C. Varni, ${ }^{55 b, 55 a}$ T. Varol, ${ }^{157}$ D. Varouchas, ${ }^{65}$ K. E. Varvell, ${ }^{156}$ M. E. Vasile, ${ }^{27 b}$ G. A. Vasquez, ${ }^{175}$ F. Vazeille, ${ }^{38}$

D. Vazquez Furelos, ${ }^{14}$ T. Vazquez Schroeder, ${ }^{36}$ J. Veatch, ${ }^{53}$ V. Vecchio, ${ }^{101}$ M. J. Veen, ${ }^{120}$ L. M. Veloce, ${ }^{166}$ F. Veloso, ${ }^{139 a, 139 c}$ S. Veneziano, ${ }^{73 a}$ A. Ventura, ${ }^{68 a, 68 b}$ A. Verbytskyi, ${ }^{115}$ V. Vercesi, ${ }^{71 a}$ M. Verducci, ${ }^{72 a, 72 b}$ C. M. Vergel Infante,${ }^{79}$ C. Vergis,${ }^{24}$

W. Verkerke, ${ }^{120}$ A. T. Vermeulen, ${ }^{120}$ J. C. Vermeulen, ${ }^{120}$ C. Vernieri, ${ }^{152}$ P. J. Verschuuren, ${ }^{94}$ M. C. Vetterli, ${ }^{151, d}$

N. Viaux Maira, ${ }^{146 \mathrm{~d}}$ T. Vickey, ${ }^{148}$ O. E. Vickey Boeriu, ${ }^{148}$ G. H. A. Viehhauser, ${ }^{134}$ L. Vigani, ${ }^{61 b}$ M. Villa, ${ }^{23 b, 23 a}$ M. Villaplana Perez, ${ }^{3}$ E. M. Villhauer, ${ }^{50}$ E. Vilucchi,${ }^{51}$ M. G. Vincter, ${ }^{34}$ G. S. Virdee, ${ }^{21}$ A. Vishwakarma, ${ }^{50}$ C. Vittori, ${ }^{23 b, 23 a}$ I. Vivarelli, ${ }^{155}$ M. Vogel, ${ }^{181}$ P. Vokac, ${ }^{141}$ S. E. von Buddenbrock,${ }^{33 \mathrm{e}}$ E. Von Toerne, ${ }^{24}$ V. Vorobel,${ }^{142}$ K. Vorobev, ${ }^{112}$ M. Vos, ${ }^{173}$ J. H. Vossebeld, ${ }^{91}$ M. Vozak, ${ }^{101}$ N. Vranjes, ${ }^{16}$ M. Vranjes Milosavljevic, ${ }^{16}$ V. Vrba, ${ }^{141}$ M. Vreeswijk,${ }^{120}$ N. K. Vu, ${ }^{102}$ R. Vuillermet, ${ }^{36}$ I. Vukotic, ${ }^{37}$ S. Wada, ${ }^{168}$ P. Wagner, ${ }^{24}$ W. Wagner, ${ }^{181}$ J. Wagner-Kuhr, ${ }^{114}$ S. Wahdan, ${ }^{181}$ H. Wahlberg,${ }^{89}$ R. Wakasa, ${ }^{168}$ V. M. Walbrecht, ${ }^{15}$ J. Walder, ${ }^{14}$ R. Walker, ${ }^{14}$ S. D. Walker, ${ }^{94}$ W. Walkowiak, ${ }^{150}$ V. Wallangen, ${ }^{45 a, 45 b}$ A. M. Wang, ${ }^{59}$ A. Z. Wang, ${ }^{180}$ C. Wang, ${ }^{60 a}$ C. Wang, ${ }^{60 \mathrm{c}}$ F. Wang, ${ }^{180} \mathrm{H}$. Wang, ${ }^{18} \mathrm{H}$. Wang, ${ }^{3} \mathrm{~J}$. Wang, ${ }^{63 \mathrm{a}} \mathrm{P}$. Wang, ${ }^{42}$ Q. Wang, ${ }^{128}$ R.-J. Wang, ${ }^{100}$ R. Wang, ${ }^{60 a}$ R. Wang, ${ }^{6}$ S. M. Wang, ${ }^{157}$ W. T. Wang, ${ }^{60}$ W. Wang, ${ }^{15 c}$ W. X. Wang, ${ }^{60 a}$ Y. Wang, ${ }^{60 a}$ Z. Wang, ${ }^{106}$ C. Wanotayaroj, ${ }^{46}$ A. Warburton, ${ }^{104}$ C. P. Ward, ${ }^{32}$ R. J. Ward, ${ }^{21}$ N. Warrack, ${ }^{57}$ A. T. Watson, ${ }^{21}$ M. F. Watson, ${ }^{21}$ G. Watts, ${ }^{147}$ B. M. Waugh, ${ }^{95}$ A. F. Webb, ${ }^{11}$ C. Weber, ${ }^{29}$ M. S. Weber, ${ }^{20}$ S. A. Weber, ${ }^{34}$ S. M. Weber, ${ }^{61 a}$ A. R. Weidberg, ${ }^{134}$ J. Weingarten, ${ }^{47}$ M. Weirich, ${ }^{100}$ C. Weiser, ${ }^{52}$ P. S. Wells, ${ }^{36}$ T. Wenaus, ${ }^{29}$ B. Wendland, ${ }^{47}$ T. Wengler, ${ }^{36}$ S. Wenig, ${ }^{36}$ N. Wermes, ${ }^{24}$ M. Wessels, ${ }^{61 a}$ T. D. Weston, ${ }^{20}$ K. Whalen, ${ }^{131}$ A. M. Wharton, ${ }^{90}$ A. S. White, ${ }^{106}$ A. White, ${ }^{8}$ M. J. White, ${ }^{1}$ D. Whiteson, ${ }^{170}$ B. W. Whitmore, ${ }^{90}$ W. Wiedenmann, ${ }^{180} \mathrm{C}$. Wiel,${ }^{48} \mathrm{M}$. Wielers, ${ }^{143} \mathrm{~N}$. Wieseotte, ${ }^{100} \mathrm{C}$. Wiglesworth, ${ }^{40}$ L. A. M. Wiik-Fuchs, ${ }^{52}$ H. G. Wilkens,${ }^{36}$ L. J. Wilkins, ${ }^{94}$ H. H. Williams, ${ }^{136}$ S. Williams, ${ }^{32}$ S. Willocq, ${ }^{103}$

P. J. Windischhofer, ${ }^{134}$ I. Wingerter-Seez, ${ }^{5}$ E. Winkels, ${ }^{155}$ F. Winklmeier, ${ }^{131}$ B. T. Winter, ${ }^{52}$ M. Wittgen, ${ }^{152}$ M. Wobisch, ${ }^{96}$

A. Wolf, ${ }^{100}$ R. Wölker, ${ }^{134}$ J. Wollrath, ${ }^{52}$ M. W. Wolter, ${ }^{85}$ H. Wolters, ${ }^{139 a, 139 c}$ V. W. S. Wong, ${ }^{174}$ N. L. Woods, ${ }^{145}$

S. D. Worm, ${ }^{46}$ B. K. Wosiek, ${ }^{85}$ K. W. Woźniak, ${ }^{85}$ K. Wraight, ${ }^{57}$ S. L. Wu, ${ }^{180}$ X. Wu, ${ }^{54}$ Y. Wu, ${ }^{60 a}$ J. Wuerzinger, ${ }^{134}$

T. R. Wyatt, ${ }^{101}$ B. M. Wynne, ${ }^{50}$ S. Xella, ${ }^{40}$ L. Xia, ${ }^{177}$ J. Xiang, ${ }^{63 c}$ X. Xiao, ${ }^{106}$ X. Xie, ${ }^{60 a}$ I. Xiotidis, ${ }^{155}$ D. Xu, ${ }^{15 a}$ H. Xu, ${ }^{60 a}$ H. Xu, ${ }^{60 a}$ L. Xu, ${ }^{29}$ T. Xu, ${ }^{144}$ W. Xu, ${ }^{106}$ Y. Xu, ${ }^{15 b}$ Z. Xu,${ }^{60 b}$ Z. Xu ${ }^{152}$ B. Yabsley, ${ }^{156}$ S. Yacoob, ${ }^{33 a}$ D. P. Yallup, ${ }^{95}$ N. Yamaguchi, ${ }^{88}$ Y. Yamaguchi, ${ }^{164}$ A. Yamamoto, ${ }^{82}$ M. Yamatani, ${ }^{162}$ T. Yamazaki, ${ }^{162}$ Y. Yamazaki, ${ }^{83}$ J. Yan ${ }^{60 c}$ Z. Yan, ${ }^{25}$ H. J. Yang, ${ }^{60 \mathrm{c}, 60 \mathrm{~d}}$ H. T. Yang, ${ }^{18}$ S. Yang, ${ }^{60 \mathrm{a}}$ T. Yang, ${ }^{63 \mathrm{c}}$ X. Yang, ${ }^{60 \mathrm{~b}, 58}$ Y. Yang, ${ }^{162}$ Z. Yang, ${ }^{60 \mathrm{a}}$ W-M. Yao, ${ }^{18}$ Y. C. Yap, ${ }^{46}$ E. Yatsenko, ${ }^{60 \mathrm{c}} \mathrm{H}$. Ye, ${ }^{15 \mathrm{c}} \mathrm{J}$. Ye, ${ }^{42}$ S. Ye ${ }^{29}$ I. Yeletskikh, ${ }^{80} \mathrm{M}$. R. Yexley ${ }^{90}$ E. Yigitbasi, ${ }^{25}$ P. Yin, ${ }^{39} \mathrm{~K}$. Yorita, ${ }^{178}$

K. Yoshihara, ${ }^{79}$ C. J. S. Young, ${ }^{36}$ C. Young, ${ }^{152}$ J. Yu, ${ }^{79}$ R. Yuan, ${ }^{60 b, o 0}$ X. Yue, ${ }^{61 a}$ M. Zaazoua, ${ }^{35 e}$ B. Zabinski, ${ }^{85}$ G. Zacharis, ${ }^{10}$ E. Zaffaroni, ${ }^{54}$ J. Zahreddine, ${ }^{135}$ A. M. Zaitsev, ${ }^{123, i}$ T. Zakareishvili, ${ }^{158 b}$ N. Zakharchuk, ${ }^{34}$ S. Zambito, ${ }^{36}$ D. Zanzi, ${ }^{36}$ S. V. Zeißner, ${ }^{47}$ C. Zeitnitz, ${ }^{181}$ G. Zemaityte, ${ }^{134}$ J. C. Zeng, ${ }^{172}$ O. Zenin, ${ }^{123}$ T. Ženišs, ${ }^{28 a}$ D. Zerwas, ${ }^{65}$ M. Zgubič,${ }^{134}$ B. Zhang, ${ }^{15 \mathrm{c}}$ D. F. Zhang, ${ }^{15 \mathrm{~b}}$ G. Zhang, ${ }^{15 \mathrm{~b}}$ J. Zhang, ${ }^{6}$ Kaili. Zhang, ${ }^{15 \mathrm{a}}$ L. Zhang, ${ }^{15 \mathrm{c}}$ L. Zhang, ${ }^{60 \mathrm{a}}$ M. Zhang, ${ }^{172}$ R. Zhang, ${ }^{180}$ S. Zhang, ${ }^{106}$ X. Zhang, ${ }^{60 \mathrm{c}}$ X. Zhang, ${ }^{60 \mathrm{~b}}$ Y. Zhang, ${ }^{15 a, 15 \mathrm{~d}}$ Z. Zhang, ${ }^{63 \mathrm{a}}$ Z. Zhang ${ }^{65}$ P. Zhao, ${ }^{49}$ Z. Zhao, ${ }^{60 \mathrm{a}}$ A. Zhemchugov ${ }^{80}$ Z. Zheng, ${ }^{106}$ D. Zhong, ${ }^{172}$ B. Zhou, ${ }^{106}$ C. Zhou, ${ }^{180}$ H. Zhou, ${ }^{7}$ M. S. Zhou, ${ }^{15 a, 15 d}$ M. Zhou, ${ }^{154}$ N. Zhou, ${ }^{60 c}$ Y. Zhou, ${ }^{7}$ C. G. Zhu, ${ }^{60 \mathrm{~b}}$ C. Zhu, ${ }^{15,15 \mathrm{~d}}$ H. L. Zhu, ${ }^{60 \mathrm{a}} \mathrm{H}$. Zhu, ${ }^{15 \mathrm{a}}$ J. Zhu, ${ }^{106}$ Y. Zhu ${ }^{60 \mathrm{a}}$ X. Zhuang, ${ }^{15 \mathrm{a}} \mathrm{K}$. Zhukov, ${ }^{111}$ V. Zhulanov, ${ }^{122 b, 122 a}$ D. Zieminska, ${ }^{66}$ N. I. Zimine, ${ }^{80}$ S. Zimmermann, ${ }^{52}$ Z. Zinonos, ${ }^{115}$ M. Ziolkowski, ${ }^{150}$ L. Živković, ${ }^{16}$ G. Zobernig, ${ }^{180}$ A. Zoccoli, ${ }^{23 b, 23 a}$ K. Zoch, ${ }^{53}$ T. G. Zorbas, ${ }^{148}$ R. Zou, ${ }^{37}$ and L. Zwalinski ${ }^{36}$

(ATLAS Collaboration)

${ }^{1}$ Department of Physics, University of Adelaide, Adelaide, Australia

${ }^{2}$ Physics Department, SUNY Albany, Albany, New York, USA

${ }^{3}$ Department of Physics, University of Alberta, Edmonton AB, Canada 


\footnotetext{
${ }^{4 a}$ Department of Physics, Ankara University, Ankara, Turkey

${ }^{4 \mathrm{~b}}$ Istanbul Aydin University, Application and Research Center for Advanced Studies, Istanbul, Turkey

${ }^{4 \mathrm{c}}$ Division of Physics, TOBB University of Economics and Technology, Ankara, Turkey

${ }^{5}$ LAPP, Université Grenoble Alpes, Université Savoie Mont Blanc, CNRS/IN2P3, Annecy, France

${ }^{6}$ High Energy Physics Division, Argonne National Laboratory, Argonne, Illinois, USA

${ }^{7}$ Department of Physics, University of Arizona, Tucson, Arizona, USA

${ }^{8}$ Department of Physics, University of Texas at Arlington, Arlington, Texas, USA

${ }^{9}$ Physics Department, National and Kapodistrian University of Athens, Athens, Greece

${ }^{10}$ Physics Department, National Technical University of Athens, Zografou, Greece

${ }^{11}$ Department of Physics, University of Texas at Austin, Austin, Texas, USA

${ }^{12 \mathrm{a}}$ Bahcesehir University, Faculty of Engineering and Natural Sciences, Istanbul, Turkey

${ }^{12 \mathrm{~b}}$ Istanbul Bilgi University, Faculty of Engineering and Natural Sciences, Istanbul, Turkey

${ }^{12 \mathrm{c}}$ Department of Physics, Bogazici University, Istanbul, Turkey

${ }^{12 \mathrm{~d}}$ Department of Physics Engineering, Gaziantep University, Gaziantep, Turkey

${ }^{13}$ Institute of Physics, Azerbaijan Academy of Sciences, Baku, Azerbaijan

${ }^{14}$ Institut de Física d'Altes Energies (IFAE), Barcelona Institute of Science and Technology, Barcelona, Spain

${ }^{15 a}$ Institute of High Energy Physics, Chinese Academy of Sciences, Beijing, China

${ }^{15 \mathrm{~b}}$ Physics Department, Tsinghua University, Beijing, China

${ }^{15 \mathrm{c}}$ Department of Physics, Nanjing University, Nanjing, China

${ }^{15 \mathrm{~d}}$ University of Chinese Academy of Science (UCAS), Beijing, China

${ }^{16}$ Institute of Physics, University of Belgrade, Belgrade, Serbia

${ }^{17}$ Department for Physics and Technology, University of Bergen, Bergen, Norway

${ }^{18}$ Physics Division, Lawrence Berkeley National Laboratory and University of California, Berkeley, California, USA

${ }^{19}$ Institut für Physik, Humboldt Universität zu Berlin, Berlin, Germany

${ }^{20}$ Albert Einstein Center for Fundamental Physics and Laboratory for High Energy Physics, University of Bern, Bern, Switzerland

${ }^{21}$ School of Physics and Astronomy, University of Birmingham, Birmingham, United Kingdom

${ }^{22 \mathrm{a}}$ Facultad de Ciencias y Centro de Investigaciónes, Universidad Antonio Nariño, Bogotá, Colombia

${ }^{22 \mathrm{~b}}$ Departamento de Física, Universidad Nacional de Colombia, Bogotá, Colombia, Colombia

${ }^{23 a}$ INFN Bologna and Universita' di Bologna, Dipartimento di Fisica, Italy

${ }^{23 \mathrm{~b}}$ INFN Sezione di Bologna, Italy

${ }^{24}$ Physikalisches Institut, Universität Bonn, Bonn, Germany

${ }^{25}$ Department of Physics, Boston University, Boston, Massachusetts, USA

${ }^{26}$ Department of Physics, Brandeis University, Waltham, Massachusetts, USA

${ }^{27 a}$ Transilvania University of Brasov, Brasov, Romania

${ }^{27 \mathrm{~b}}$ Horia Hulubei National Institute of Physics and Nuclear Engineering, Bucharest, Romania

${ }^{27 c}$ Department of Physics, Alexandru Ioan Cuza University of Iasi, Iasi, Romania

${ }^{27 \mathrm{~d}}$ National Institute for Research and Development of Isotopic and Molecular Technologies, Physics Department, Cluj-Napoca, Romania

${ }^{27 e}$ University Politehnica Bucharest, Bucharest, Romania

${ }^{27 \mathrm{f}}$ West University in Timisoara, Timisoara, Romania

${ }^{28 a}$ Faculty of Mathematics, Physics and Informatics, Comenius University, Bratislava, Slovak Republic

${ }^{28 \mathrm{~b}}$ Department of Subnuclear Physics, Institute of Experimental Physics of the Slovak Academy of Sciences,

Kosice, Slovak Republic

${ }^{29}$ Physics Department, Brookhaven National Laboratory, Upton, New York, USA

${ }^{30}$ Departamento de Física, Universidad de Buenos Aires, Buenos Aires, Argentina

${ }^{31}$ California State University, California, USA

${ }^{32}$ Cavendish Laboratory, University of Cambridge, Cambridge, United Kingdom

${ }^{33 a}$ Department of Physics, University of Cape Town, Cape Town, South Africa

${ }^{33 \mathrm{~b}}$ iThemba Labs, Western Cape, South Africa

${ }^{33 \mathrm{c}}$ Department of Mechanical Engineering Science, University of Johannesburg, Johannesburg, South Africa

${ }^{33 \mathrm{~d}}$ University of South Africa, Department of Physics, Pretoria, South Africa

${ }^{33 \mathrm{e}}$ School of Physics, University of the Witwatersrand, Johannesburg, South Africa

${ }^{34}$ Department of Physics, Carleton University, Ottawa ON, Canada

${ }^{35 a}$ Faculté des Sciences Ain Chock, Réseau Universitaire de Physique des Hautes Energies-Université Hassan II, Casablanca, Morocco

${ }^{35 \mathrm{~b}}$ Faculté des Sciences, Université Ibn-Tofail, Kénitra, Morocco
} 
${ }^{35 \mathrm{c}}$ Faculté des Sciences Semlalia, Université Cadi Ayyad, LPHEA-Marrakech, Morocco

${ }^{35 \mathrm{~d}}$ Faculté des Sciences, Université Mohamed Premier and LPTPM, Oujda, Morocco

${ }^{35 \mathrm{e}}$ Faculté des sciences, Université Mohammed V, Rabat, Morocco

${ }^{36}$ CERN, Geneva, Switzerland

${ }^{37}$ Enrico Fermi Institute, University of Chicago, Chicago, Illinois, USA

${ }^{38}$ LPC, Université Clermont Auvergne, CNRS/IN2P3, Clermont-Ferrand, France

${ }^{39}$ Nevis Laboratory, Columbia University, Irvington, New York, USA

${ }^{40}$ Niels Bohr Institute, University of Copenhagen, Copenhagen, Denmark

${ }^{41 a}$ Dipartimento di Fisica, Università della Calabria, Rende, Italy

${ }^{41 \mathrm{~b}}$ INFN Gruppo Collegato di Cosenza, Laboratori Nazionali di Frascati, Italy

${ }^{42}$ Physics Department, Southern Methodist University, Dallas, Texas, USA

${ }^{43}$ Physics Department, University of Texas at Dallas, Richardson, Texas, USA

${ }^{44}$ National Centre for Scientific Research “Demokritos”, Agia Paraskevi, Greece

${ }^{45 a}$ Department of Physics, Stockholm University, Sweden

${ }^{45 \mathrm{~b}}$ Oskar Klein Centre, Stockholm, Sweden

${ }^{46}$ Deutsches Elektronen-Synchrotron DESY, Hamburg and Zeuthen, Germany

${ }^{47}$ Lehrstuhl für Experimentelle Physik IV, Technische Universität Dortmund, Dortmund, Germany

${ }^{48}$ Institut für Kern- und Teilchenphysik, Technische Universität Dresden, Dresden, Germany

${ }^{49}$ Department of Physics, Duke University, Durham, North Carolina, USA

${ }^{50}$ SUPA-School of Physics and Astronomy, University of Edinburgh, Edinburgh, United Kingdom

${ }^{51}$ INFN e Laboratori Nazionali di Frascati, Frascati, Italy

${ }^{52}$ Physikalisches Institut, Albert-Ludwigs-Universität Freiburg, Freiburg, Germany

${ }^{53}$ II. Physikalisches Institut, Georg-August-Universität Göttingen, Göttingen, Germany

${ }^{54}$ Département de Physique Nucléaire et Corpusculaire, Université de Genève, Genève, Switzerland

${ }^{55}$ Dipartimento di Fisica, Università di Genova, Genova, Italy

${ }^{55 \mathrm{~b}}$ INFN Sezione di Genova, Italy

${ }^{56}$ II. Physikalisches Institut, Justus-Liebig-Universität Giessen, Giessen, Germany

${ }^{57}$ SUPA-School of Physics and Astronomy, University of Glasgow, Glasgow, United Kingdom

${ }^{58}$ LPSC, Université Grenoble Alpes, CNRS/IN2P3, Grenoble INP, Grenoble, France

${ }^{59}$ Laboratory for Particle Physics and Cosmology, Harvard University, Cambridge, Massachusetts, USA

${ }^{60 \mathrm{a}}$ Department of Modern Physics and State Key Laboratory of Particle Detection and Electronics,

University of Science and Technology of China, Hefei, China

${ }^{60 \mathrm{~b}}$ Institute of Frontier and Interdisciplinary Science and Key Laboratory of Particle Physics and Particle Irradiation (MOE), Shandong University, Qingdao, China

${ }^{60 \mathrm{c}}$ School of Physics and Astronomy, Shanghai Jiao Tong University, KLPPAC-MoE, SKLPPC, Shanghai, China

${ }^{60 \mathrm{~d}}$ Tsung-Dao Lee Institute, Shanghai, China

${ }^{61 a}$ Kirchhoff-Institut für Physik, Ruprecht-Karls-Universität Heidelberg, Heidelberg, Germany

${ }^{61 \mathrm{~b}}$ Physikalisches Institut, Ruprecht-Karls-Universität Heidelberg, Heidelberg, Germany

${ }^{62}$ Faculty of Applied Information Science, Hiroshima Institute of Technology, Hiroshima, Japan

${ }^{63 a}$ Department of Physics, Chinese University of Hong Kong, Shatin, N.T., Hong Kong, China

${ }^{63 \mathrm{~b}}$ Department of Physics, University of Hong Kong, Hong Kong, China

${ }^{63 \mathrm{c}}$ Department of Physics and Institute for Advanced Study, Hong Kong University of Science and

Technology, Clear Water Bay, Kowloon, Hong Kong, China

${ }^{64}$ Department of Physics, National Tsing Hua University, Hsinchu, Taiwan

${ }^{65}$ IJCLab, Université Paris-Saclay, CNRS/IN2P3, 91405, Orsay, France

${ }^{66}$ Department of Physics, Indiana University, Bloomington, Indiana, USA

${ }^{67 a}$ INFN Gruppo Collegato di Udine, Sezione di Trieste, Udine, Italy

${ }^{67 \mathrm{~b}}$ ICTP, Trieste, Italy

${ }^{67 \mathrm{c}}$ Dipartimento Politecnico di Ingegneria e Architettura, Università di Udine, Udine, Italy

${ }^{68 \mathrm{a}}$ INFN Sezione di Lecce, Italy

${ }^{68 \mathrm{~b}}$ Dipartimento di Matematica e Fisica, Università del Salento, Lecce, Italy

${ }^{69 a}$ INFN Sezione di Milano, Italy

${ }^{69 \mathrm{~b}}$ Dipartimento di Fisica, Università di Milano, Milano, Italy

${ }^{70 a}$ INFN Sezione di Napoli, Italy

${ }^{70 \mathrm{~b}}$ Dipartimento di Fisica, Università di Napoli, Napoli, Italy

${ }^{71 a}$ INFN Sezione di Pavia, Italy

${ }^{71 b}$ Dipartimento di Fisica, Università di Pavia, Pavia, Italy

${ }^{72 a}$ INFN Sezione di Pisa, Italy

${ }^{72 b}$ Dipartimento di Fisica E. Fermi, Università di Pisa, Pisa, Italy 


\author{
${ }^{73 a}$ INFN Sezione di Roma, Italy \\ ${ }^{73 b}$ Dipartimento di Fisica, Sapienza Università di Roma, Roma, Italy \\ ${ }^{74 a}$ INFN Sezione di Roma Tor Vergata, Italy \\ ${ }^{74 b}$ Dipartimento di Fisica, Università di Roma Tor Vergata, Roma, Italy \\ ${ }^{75 a}$ INFN Sezione di Roma Tre, Italy \\ ${ }^{75 b}$ Dipartimento di Matematica e Fisica, Università Roma Tre, Roma, Italy \\ ${ }^{76 a}$ INFN-TIFPA, Italy \\ ${ }^{76 \mathrm{~b}}$ Università degli Studi di Trento, Trento, Italy \\ ${ }^{77}$ Institut für Astro- und Teilchenphysik, Leopold-Franzens-Universität, Innsbruck, Austria \\ ${ }^{78}$ University of Iowa, Iowa City, Iowa, USA \\ ${ }^{79}$ Department of Physics and Astronomy, Iowa State University, Ames, Iowa, USA \\ ${ }^{80}$ Joint Institute for Nuclear Research, Dubna, Russia \\ ${ }^{81 a}$ Departamento de Engenharia Elétrica, Universidade Federal de Juiz de Fora (UFJF), \\ Juiz de Fora, Brazil \\ ${ }^{81 \mathrm{~b}}$ Universidade Federal do Rio De Janeiro COPPE/EE/IF, Rio de Janeiro, Brazil \\ ${ }^{81 \mathrm{c}}$ Universidade Federal de São João del Rei (UFSJ), São João del Rei, Brazil \\ ${ }^{81 \mathrm{~d}}$ Instituto de Física, Universidade de São Paulo, São Paulo, Brazil \\ ${ }^{82}$ KEK, High Energy Accelerator Research Organization, Tsukuba, Japan \\ ${ }^{83}$ Graduate School of Science, Kobe University, Kobe, Japan \\ ${ }^{84 \mathrm{a}}$ AGH University of Science and Technology, Faculty of Physics and Applied Computer Science, \\ Krakow, Poland \\ ${ }^{84 \mathrm{~b}}$ Marian Smoluchowski Institute of Physics, Jagiellonian University, Krakow, Poland \\ ${ }^{85}$ Institute of Nuclear Physics Polish Academy of Sciences, Krakow, Poland \\ ${ }^{86}$ Faculty of Science, Kyoto University, Kyoto, Japan \\ ${ }^{87}$ Kyoto University of Education, Kyoto, Japan \\ ${ }^{88}$ Research Center for Advanced Particle Physics and Department of Physics, Kyushu University, \\ Fukuoka, Japan \\ ${ }^{89}$ Instituto de Física La Plata, Universidad Nacional de La Plata and CONICET, La Plata, Argentina \\ ${ }^{90}$ Physics Department, Lancaster University, Lancaster, United Kingdom \\ ${ }^{91}$ Oliver Lodge Laboratory, University of Liverpool, Liverpool, United Kingdom \\ ${ }^{92}$ Department of Experimental Particle Physics, Jožef Stefan Institute and Department of Physics, \\ University of Ljubljana, Ljubljana, Slovenia \\ ${ }^{93}$ School of Physics and Astronomy, Queen Mary University of London, London, United Kingdom \\ ${ }^{94}$ Department of Physics, Royal Holloway University of London, Egham, United Kingdom \\ ${ }^{95}$ Department of Physics and Astronomy, University College London, London, United Kingdom \\ ${ }^{96}$ Louisiana Tech University, Ruston, Louisiana, USA \\ ${ }^{97}$ Fysiska institutionen, Lunds universitet, Lund, Sweden \\ ${ }^{98}$ Centre de Calcul de l'Institut National de Physique Nucléaire et de Physique des Particules (IN2P3), \\ Villeurbanne, France \\ ${ }^{99}$ Departamento de Física Teorica C-15 and CIAFF, Universidad Autónoma de Madrid, Madrid, Spain \\ ${ }^{100}$ Institut für Physik, Universität Mainz, Mainz, Germany \\ ${ }^{101}$ School of Physics and Astronomy, University of Manchester, Manchester, United Kingdom \\ ${ }^{102}$ CPPM, Aix-Marseille Université, CNRS/IN2P3, Marseille, France \\ ${ }^{103}$ Department of Physics, University of Massachusetts, Amherst, Massachusetts, USA \\ ${ }^{104}$ Department of Physics, McGill University, Montreal QC, Canada \\ ${ }^{105}$ School of Physics, University of Melbourne, Victoria, Australia \\ ${ }^{106}$ Department of Physics, University of Michigan, Ann Arbor, Michigan, USA \\ ${ }^{107}$ Department of Physics and Astronomy, Michigan State University, East Lansing, Michigan, USA \\ ${ }^{108}$ B.I. Stepanov Institute of Physics, National Academy of Sciences of Belarus, Minsk, Belarus \\ ${ }^{109}$ Research Institute for Nuclear Problems of Byelorussian State University, Minsk, Belarus \\ ${ }^{110}$ Group of Particle Physics, University of Montreal, Montreal QC, Canada \\ ${ }^{111}$ P.N. Lebedev Physical Institute of the Russian Academy of Sciences, Moscow, Russia \\ ${ }^{112}$ National Research Nuclear University MEPhI, Moscow, Russia \\ ${ }^{113}$ D.V. Skobeltsyn Institute of Nuclear Physics, M.V. Lomonosov Moscow State University, \\ Moscow, Russia \\ ${ }^{114}$ Fakultät für Physik, Ludwig-Maximilians-Universität München, München, Germany \\ ${ }^{115}$ Max-Planck-Institut für Physik (Werner-Heisenberg-Institut), München, Germany \\ ${ }^{116}$ Nagasaki Institute of Applied Science, Nagasaki, Japan \\ ${ }^{117}$ Graduate School of Science and Kobayashi-Maskawa Institute, Nagoya University, Nagoya, Japan \\ ${ }^{118}$ Department of Physics and Astronomy, University of New Mexico, Albuquerque, New Mexico, USA
}


${ }^{119}$ Institute for Mathematics, Astrophysics and Particle Physics, Radboud University Nijmegen/Nikhef, Nijmegen, Netherlands

${ }^{120}$ Nikhef National Institute for Subatomic Physics and University of Amsterdam, Amsterdam, Netherlands ${ }^{121}$ Department of Physics, Northern Illinois University, DeKalb, Illinois, USA

${ }^{122 \mathrm{a}}$ Budker Institute of Nuclear Physics and NSU, SB RAS, Novosibirsk, Russia

${ }^{122 \mathrm{~b}}$ Novosibirsk State University Novosibirsk, Russia

${ }^{123}$ Institute for High Energy Physics of the National Research Centre Kurchatov Institute, Protvino, Russia

${ }^{124}$ Institute for Theoretical and Experimental Physics named by A.I. Alikhanov of National Research Centre "Kurchatov Institute", Moscow, Russia

${ }^{125}$ Department of Physics, New York University, New York, New York, USA

${ }^{126}$ Ochanomizu University, Otsuka, Bunkyo-ku, Tokyo, Japan

${ }^{127}$ Ohio State University, Columbus, Ohio, USA

${ }^{128}$ Homer L. Dodge Department of Physics and Astronomy, University of Oklahoma, Norman, Oklahoma, USA

${ }^{129}$ Department of Physics, Oklahoma State University, Stillwater, Oklahoma, USA

${ }^{130}$ Palacký University, RCPTM, Joint Laboratory of Optics, Olomouc, Czech Republic

${ }^{131}$ Institute for Fundamental Science, University of Oregon, Eugene, Oregon, USA

${ }^{132}$ Graduate School of Science, Osaka University, Osaka, Japan

${ }^{133}$ Department of Physics, University of Oslo, Oslo, Norway

${ }^{134}$ Department of Physics, Oxford University, Oxford, United Kingdom

${ }^{135}$ LPNHE, Sorbonne Université, Université de Paris, CNRS/IN2P3, Paris, France

${ }^{136}$ Department of Physics, University of Pennsylvania, Philadelphia, Pennsylvania, USA

${ }^{137}$ Konstantinov Nuclear Physics Institute of National Research Centre "Kurchatov Institute", PNPI, St. Petersburg, Russia

${ }^{138}$ Department of Physics and Astronomy, University of Pittsburgh, Pittsburgh, Pennsylvania, USA

${ }^{139 a}$ Laboratório de Instrumentação e Física Experimental de Partículas-LIP, Lisboa, Portugal

${ }^{139 b}$ Departamento de Física, Faculdade de Ciências, Universidade de Lisboa, Lisboa, Portugal

${ }^{139 \mathrm{c}}$ Departamento de Física, Universidade de Coimbra, Coimbra, Portugal

${ }^{139 \mathrm{~d}}$ Centro de Física Nuclear da Universidade de Lisboa, Lisboa, Portugal

${ }^{139 \mathrm{e}}$ Departamento de Física, Universidade do Minho, Braga, Portugal

${ }^{139 \mathrm{f}}$ Departamento de Física Teórica y del Cosmos, Universidad de Granada, Granada (Spain), Spain

${ }^{139 g}$ Dep Física and CEFITEC of Faculdade de Ciências e Tecnologia, Universidade Nova de Lisboa, Caparica, Portugal

${ }^{139 h}$ Instituto Superior Técnico, Universidade de Lisboa, Lisboa, Portugal

${ }^{140}$ Institute of Physics of the Czech Academy of Sciences, Prague, Czech Republic

${ }^{141}$ Czech Technical University in Prague, Prague, Czech Republic

${ }^{142}$ Charles University, Faculty of Mathematics and Physics, Prague, Czech Republic

${ }^{143}$ Particle Physics Department, Rutherford Appleton Laboratory, Didcot, United Kingdom

${ }^{144}$ IRFU, CEA, Université Paris-Saclay, Gif-sur-Yvette, France

${ }^{145}$ Santa Cruz Institute for Particle Physics, University of California Santa Cruz, Santa Cruz, California, USA

${ }^{146 a}$ Departamento de Física, Pontificia Universidad Católica de Chile, Santiago, Chile

${ }^{146 \mathrm{~b}}$ Universidad Andres Bello, Department of Physics, Santiago, Chile

${ }^{146 c}$ Instituto de Alta Investigación, Universidad de Tarapacá, Chile

${ }^{146 \mathrm{~d}}$ Departamento de Física, Universidad Técnica Federico Santa María, Valparaíso, Chile

${ }^{147}$ Department of Physics, University of Washington, Seattle, Washington, USA

${ }^{148}$ Department of Physics and Astronomy, University of Sheffield, Sheffield, United Kingdom

${ }^{149}$ Department of Physics, Shinshu University, Nagano, Japan

${ }^{150}$ Department Physik, Universität Siegen, Siegen, Germany

${ }^{151}$ Department of Physics, Simon Fraser University, Burnaby BC, Canada

${ }^{152}$ SLAC National Accelerator Laboratory, Stanford, California, USA

${ }^{153}$ Physics Department, Royal Institute of Technology, Stockholm, Sweden

${ }^{154}$ Departments of Physics and Astronomy, Stony Brook University, Stony Brook, New York, USA

${ }^{155}$ Department of Physics and Astronomy, University of Sussex, Brighton, United Kingdom

${ }^{156}$ School of Physics, University of Sydney, Sydney, Australia

${ }^{157}$ Institute of Physics, Academia Sinica, Taipei, Taiwan

${ }^{158 \mathrm{a}}$ E. Andronikashvili Institute of Physics, Iv. Javakhishvili Tbilisi State University, Tbilisi, Georgia

${ }^{158 b}$ High Energy Physics Institute, Tbilisi State University, Tbilisi, Georgia

${ }^{159}$ Department of Physics, Technion, Israel Institute of Technology, Haifa, Israel

${ }^{160}$ Raymond and Beverly Sackler School of Physics and Astronomy, Tel Aviv University, Tel Aviv, Israel 


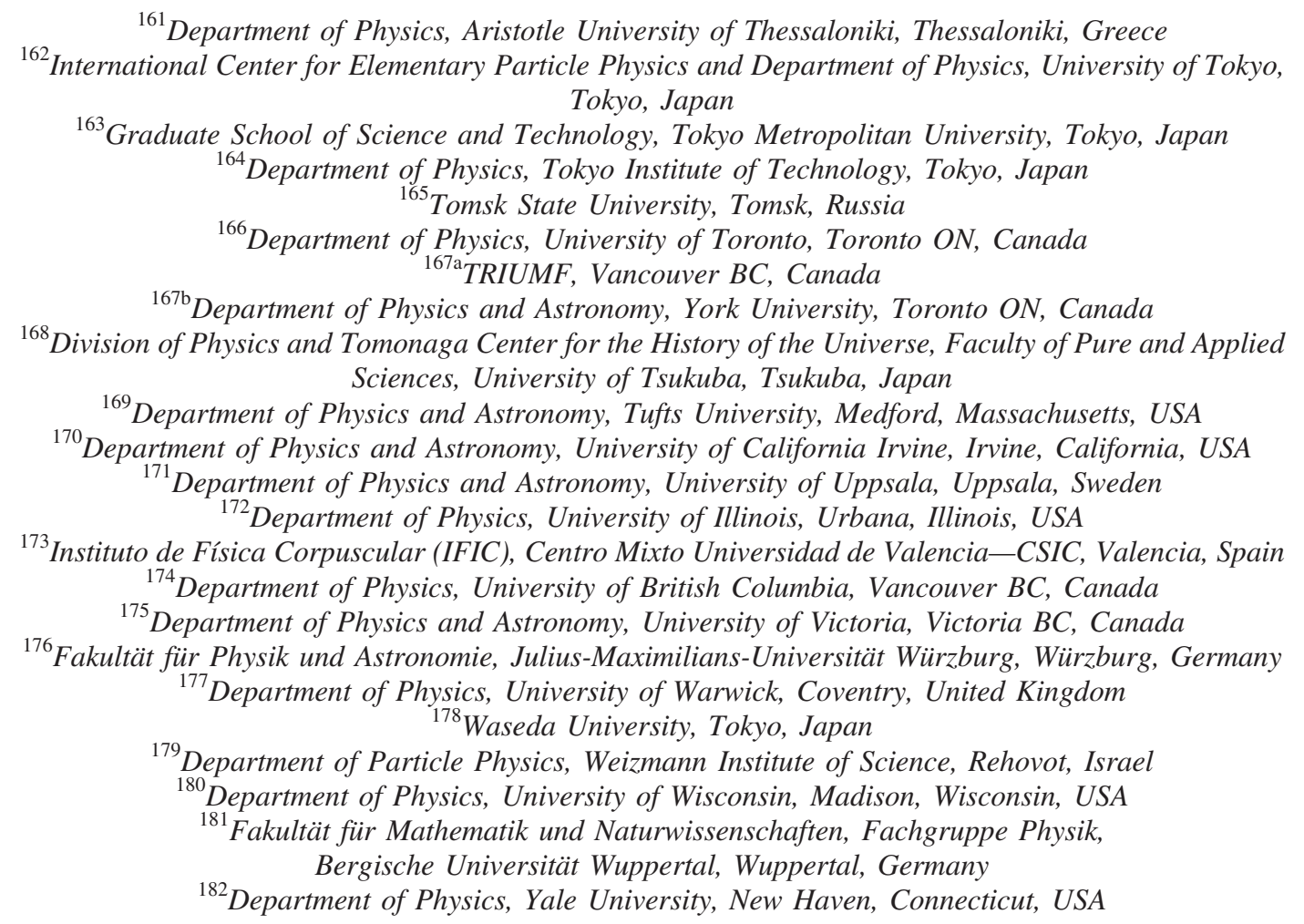

${ }^{\mathrm{a}}$ Deceased.

${ }^{b}$ Also at Department of Physics, King's College London, London, United Kingdom.

${ }^{\mathrm{c}}$ Also at Instituto de Fisica Teorica, IFT-UAM/CSIC, Madrid, Spain.

${ }^{\mathrm{d}}$ Also at TRIUMF, Vancouver BC, Canada.

${ }^{\mathrm{e}}$ Also at Department of Physics and Astronomy, University of Louisville, Louisville, Kentucky, USA.

${ }^{\mathrm{f}}$ Also at Physics Department, An-Najah National University, Nablus, Palestine.

${ }^{g}$ Also at Department of Physics, University of Fribourg, Fribourg, Switzerland.

${ }^{\mathrm{h}}$ Also at Departament de Fisica de la Universitat Autonoma de Barcelona, Barcelona, Spain.

${ }^{\mathrm{i}}$ Also at Moscow Institute of Physics and Technology State University, Dolgoprudny, Russia.

${ }^{\mathrm{j}}$ Also at Department of Physics, Ben Gurion University of the Negev, Beer Sheva, Israel.

${ }^{\mathrm{k}}$ Also at Universita di Napoli Parthenope, Napoli, Italy.

${ }^{1}$ Also at Institute of Particle Physics (IPP), Vancouver, Canada.

${ }^{\mathrm{m}}$ Also at Department of Physics, St. Petersburg State Polytechnical University, St. Petersburg, Russia.

${ }^{\mathrm{n}}$ Also at Borough of Manhattan Community College, City University of New York, New York, New York, USA.

${ }^{\circ}$ Also at Department of Physics, California State University, Fresno, USA.

${ }^{\mathrm{p}}$ Also at Department of Financial and Management Engineering, University of the Aegean, Chios, Greece.

${ }^{\mathrm{q}}$ Also at Centro Studi e Ricerche Enrico Fermi, Italy.

${ }^{\mathrm{r}}$ Also at Department of Physics, California State University, East Bay, USA.

${ }^{\mathrm{s}}$ Also at Institucio Catalana de Recerca i Estudis Avancats, ICREA, Barcelona, Spain.

${ }^{t}$ Also at IJCLab, Université Paris-Saclay, CNRS/IN2P3, 91405, Orsay, France.

${ }^{\mathrm{u}}$ Also at Graduate School of Science, Osaka University, Osaka, Japan.

${ }^{v}$ Also at Physikalisches Institut, Albert-Ludwigs-Universität Freiburg, Freiburg, Germany.

${ }^{\mathrm{w}}$ Also at University of Chinese Academy of Sciences (UCAS), Beijing, China.

${ }^{\mathrm{x}}$ Also at Institute of Physics, Azerbaijan Academy of Sciences, Baku, Azerbaijan.

${ }^{y}$ Also at Institute for Mathematics, Astrophysics and Particle Physics, Radboud University Nijmegen/Nikhef, Nijmegen, Netherlands.

${ }^{\mathrm{z}}$ Also at CERN, Geneva, Switzerland.

${ }^{\text {aa }}$ Also at Joint Institute for Nuclear Research, Dubna, Russia.

${ }^{\mathrm{bb}}$ Also at Hellenic Open University, Patras, Greece.

${ }^{c c}$ Also at The City College of New York, New York. New York, USA.

${ }^{\mathrm{dd}}$ Also at Dipartimento di Matematica, Informatica e Fisica, Università di Udine, Udine, Italy.

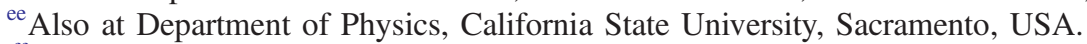

${ }^{\mathrm{ff}}$ Also at Département de Physique Nucléaire et Corpusculaire, Université de Genève, Genève, Switzerland. 
${ }^{\mathrm{gg}}$ Also at Louisiana Tech University, Ruston, Louisiana, USA.

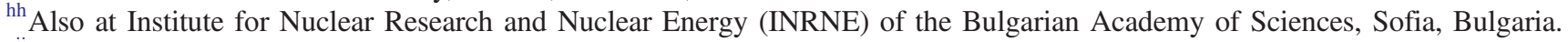

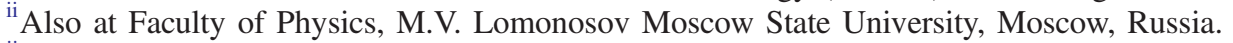

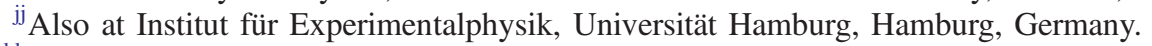

${ }^{\mathrm{kk}}$ Also at CPPM, Aix-Marseille Université, CNRS/IN2P3, Marseille, France.

${ }^{11}$ Also at National Research Nuclear University MEPhI, Moscow, Russia.

${ }^{\mathrm{mm}}$ Also at Institute for Particle and Nuclear Physics, Wigner Research Centre for Physics, Budapest, Hungary.

${ }^{n n}$ Also at Giresun University, Faculty of Engineering, Giresun, Turkey.

${ }^{\text {oo }}$ Also at Department of Physics and Astronomy, Michigan State University, East Lansing, Michigan, USA. 